\title{
Synthesis and Photophysical Behavior of a Highly Fluorescent Family of Unsymmetrical Organoboron Complexes Containing 5-(Pyridin-2- ylmethylene)imidazolidine-2,4-dione Moieties
}

M. Soledad Garre, ${ }^{[a]}$ Raúl Losantos, ${ }^{[b]}$ Sara Gutiérrez, ${ }^{[a]}$ David Sucunza, ${ }^{*[a]}$ Patricia García-García, ${ }^{[a]}$ Diego Sampedro*[b] and Juan J. Vaquero*[a]

[a] Departamento de Química Orgánica y Química Inorgánica, Instituto de Investigación Química “Andrés M. del Río" (IQAR), Universidad de Alcalá, IRYCIS, Edificio de Farmacia, E-28805, Alcalá de Henares, Spain

[b] Departamento de Química, Centro de Investigación en Síntesis Química (CISQ), Universidad de La Rioja, Madre de Dios, 53, E-26oo6, Logroño, Spain

Corresponding Authors: david.sucunza@uah.es; diego.sampedro@unirioja.es;

juanjose.vaquero@uah.es

\section{Electronic Supplementary Information}

\section{Table of Contents}

Optical properties 


\section{Optical properties}

The luminescence properties of all compounds were measured using $c a .5 \times 10^{-5} \mathrm{M}$ solutions of each compound in the solvent of choice, acetonitrile or water. The excitation and emission spectra were recorded using a Shimadzu RF6000 spectrofluorimeter. Then, the fluorescence quantum yield was recorded using quinine solutions with a known quantum yield of $\phi_{360 \mathrm{~nm}}=0.6$ through a linear regression between integrated emission vs. absorbance at $360 \mathrm{~nm}$ and comparing the slopes to get the quantum yield of the measured molecule.

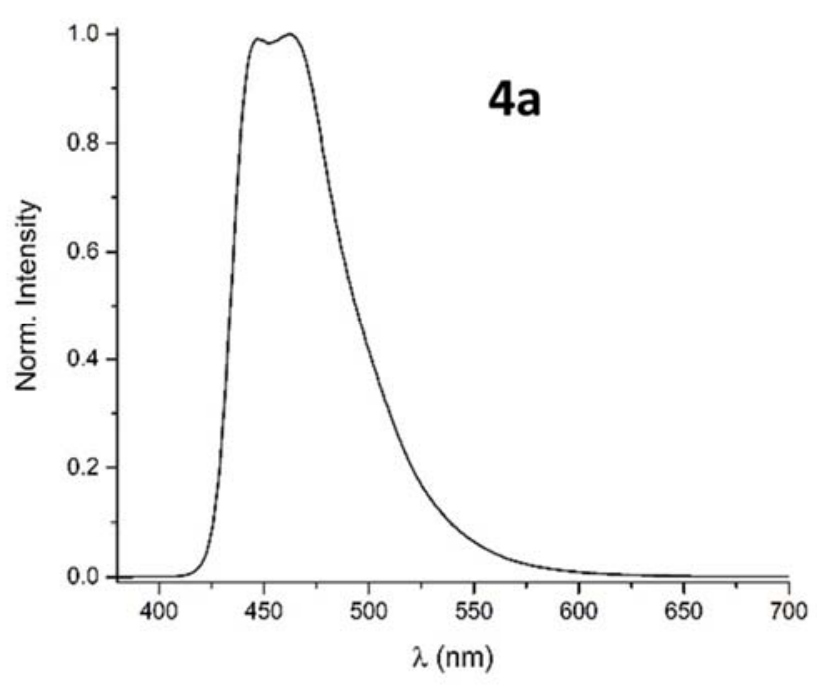

Figure S1. 4a emission spectrum.

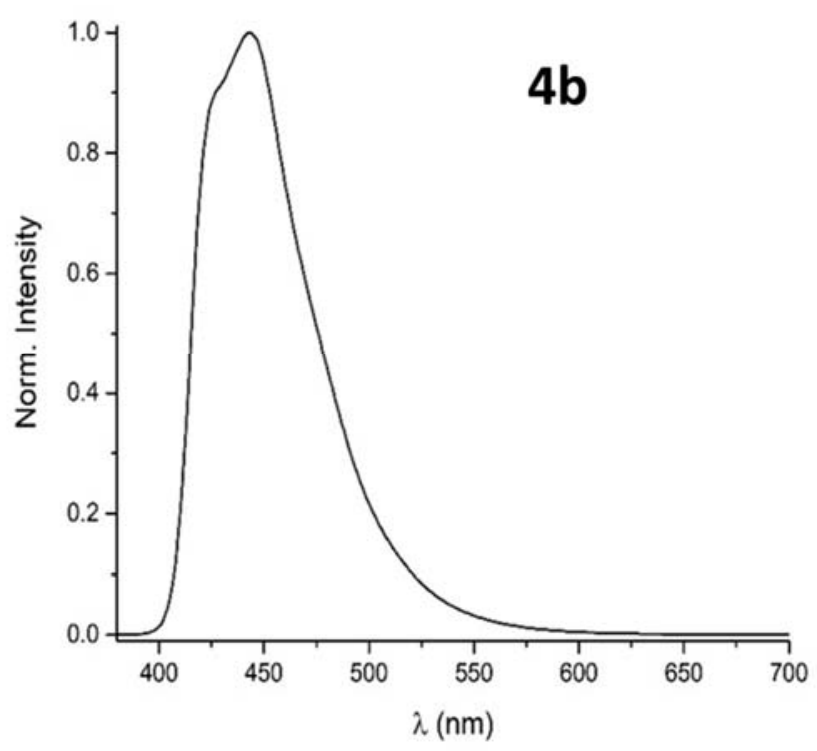

Figure S2. 4b emission spectrum. 


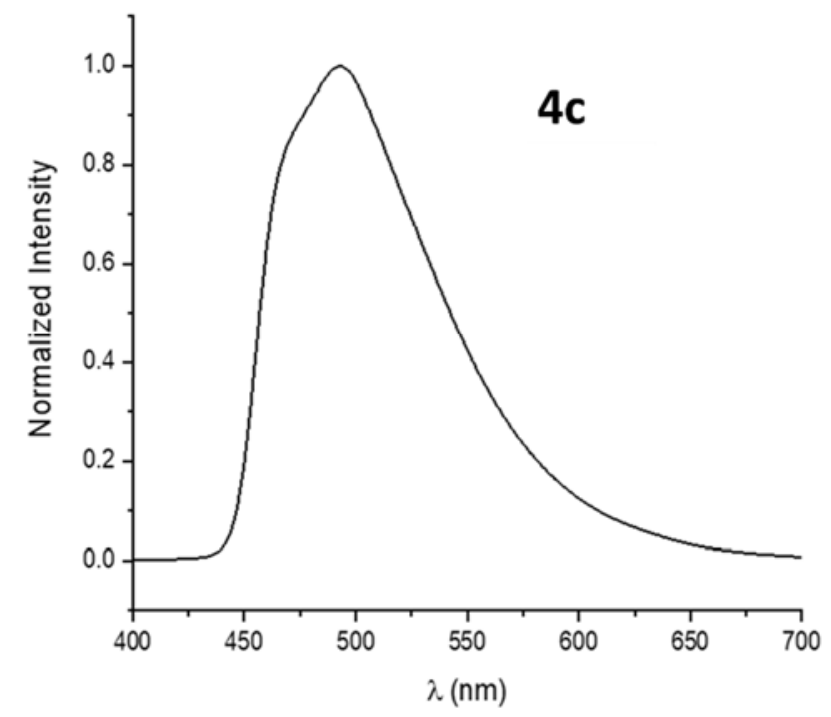

Figure S3. 4c emission spectrum.

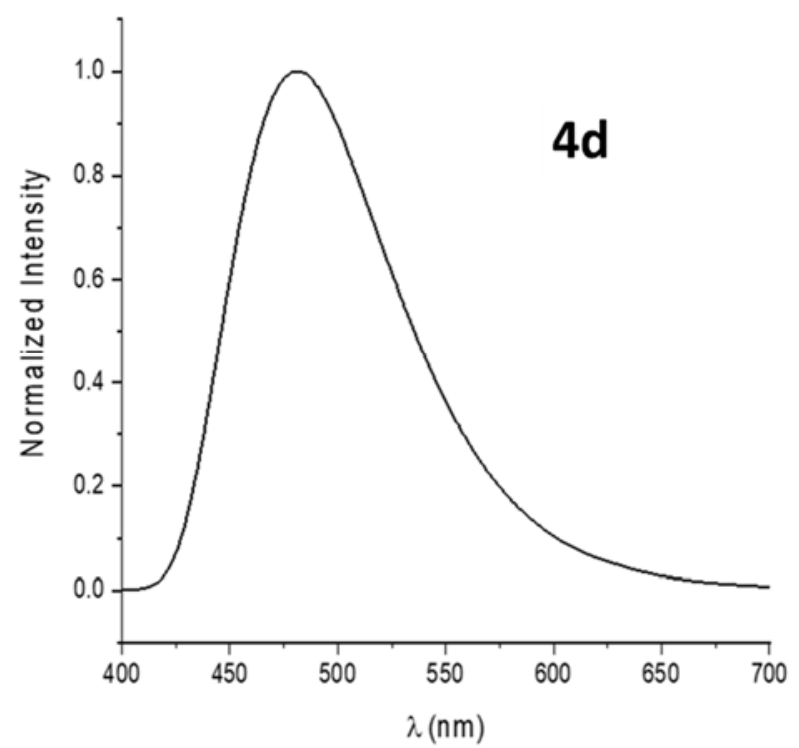

Figure S4. 4d emission spectrum. 


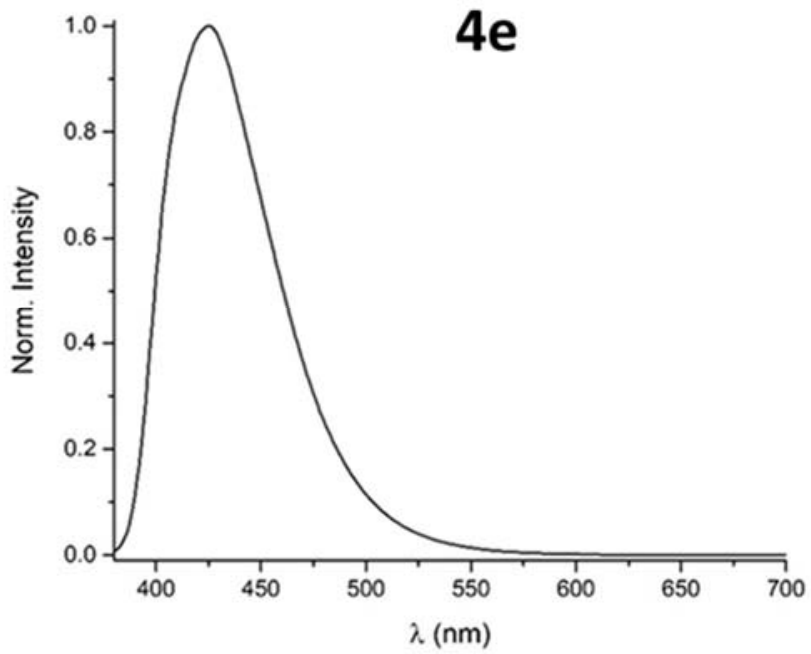

Figure S5. 4e emission spectrum.

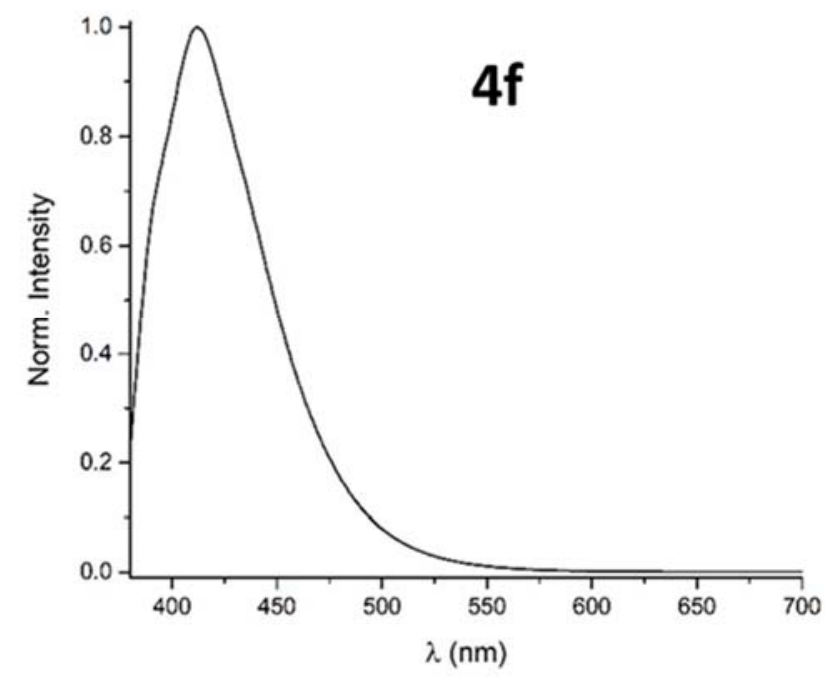

Figure $\mathbf{S 6 .} \mathbf{4 f}$ emission spectrum. 


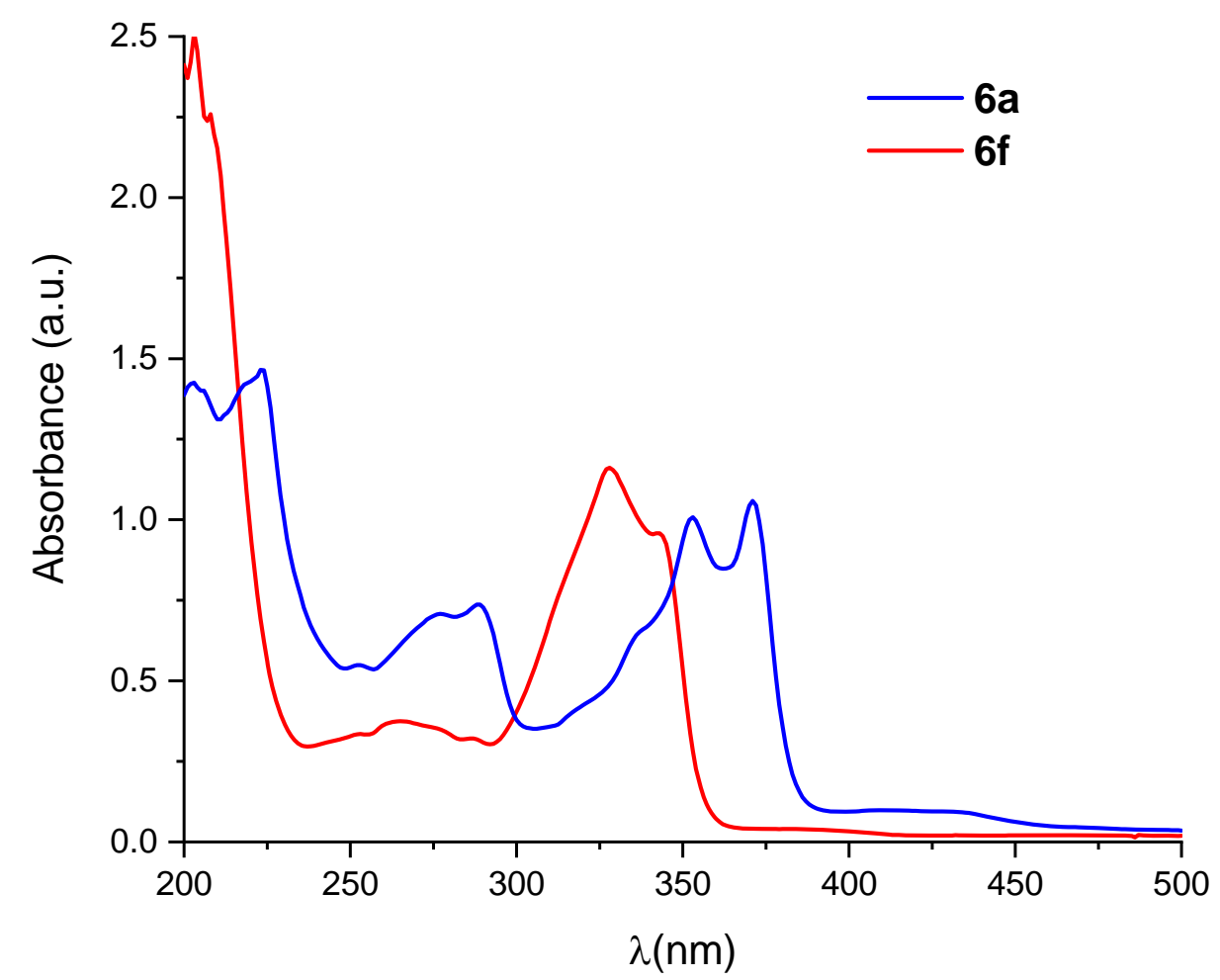

Figure S7. Absorption spectra for $6 \mathbf{a}$ and $\mathbf{6 f}$.

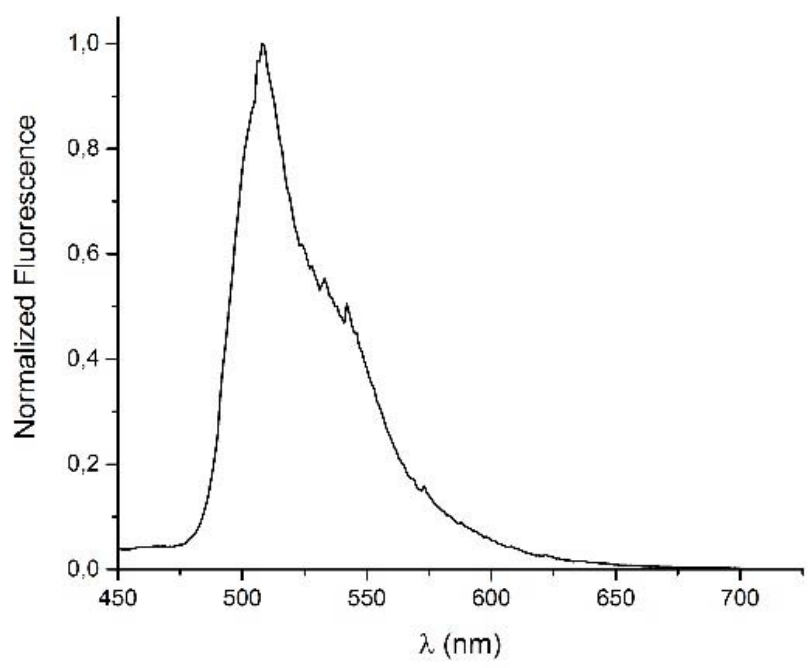

Figure S8. 6a emission spectrum. Excitation at different wavelengths (276, 290, 335, 353 y 371 $\mathrm{nm}$, see absorption spectrum) lead to the same emissive state with very low quantum yield. 


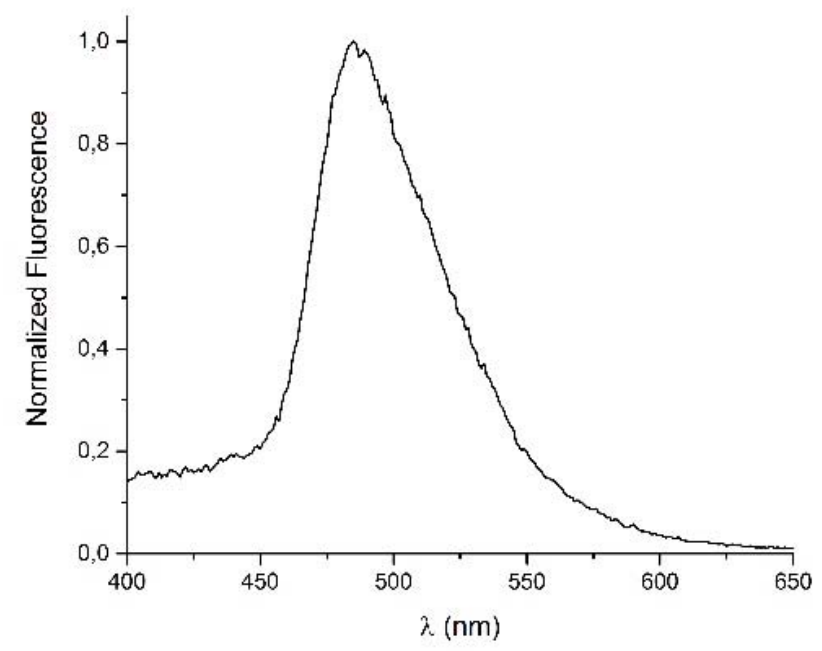

Figure S9. 6 f emission spectrum. Excitation at different wavelengths (328 y $342 \mathrm{~nm}$, see absorption spectrum) lead to the same emissive state with very low quantum yield.

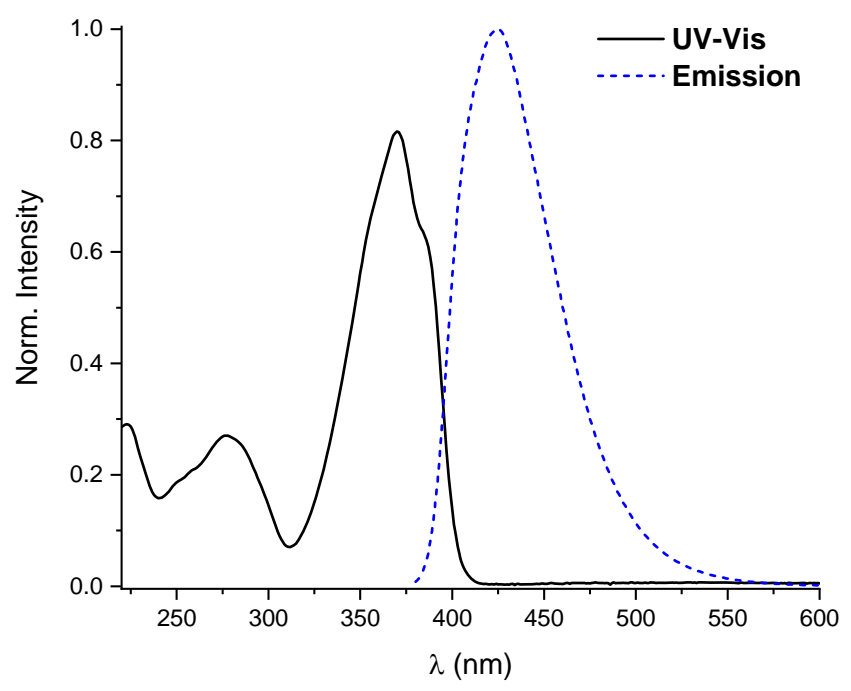

Figure S10. Optical properties of 9. Excitation at different wavelengths ( 360 y $380 \mathrm{~nm}$ ) lead to the same emissive state with quantum yield $\phi_{f}=0.72$.

\section{Quantum yield determination}

The most reliable method for recording $\Phi_{\mathrm{F}}$ without an integrating sphere is the comparative method of Williams et al. (A. T. R. Williams, S. A. Winfield and J. N. Miller. Analyst, 1983, 108, 1067), which involves the use of well characterized standard samples with known $\Phi_{\mathrm{F}}$ values. Essentially, solutions of the standard and test samples with identical absorbance at the same excitation wavelength can be assumed to be absorbing the same number of photons. Hence, a simple ratio of the integrated fluorescence intensities of the two solutions (recorded under identical conditions) will yield the ratio of the quantum yield values. Since $\Phi_{\mathrm{F}}$ for the standard sample is known, it is trivial to calculate the $\Phi_{\mathrm{F}}$ for the test sample. 
The quantum yield measurements were done according to the described methodology using quinine sulfate in $0.5 \mathrm{M} \mathrm{H}_{2} \mathrm{SO}_{4}$ as standard. Quinine sulfate dihydrate, purity: $99+\%$, was obtained from ACROS Organics and used without further purification. The reference $Q Y$ value of $\Phi_{f}=0.60$ was used according to updated values in recent literature (K. Suzuki, A. Kobayashi, S. Kaneko, K. Takehira, T. Yoshihara, H. Ishida, Y. Shiina, S. Oishic, S. Tobita. Phys. Chem. Chem. Phys. 2009, 11, 9850).

The fluorescence spectra were recorded using $10 \mathrm{~mm}$ quartz cuvettes and HPLC grade solvents using a Shimadzu RF600 spectrofluorimeter, exciting at $360 \mathrm{~nm}$ and using slit sizes of $5 \mathrm{~nm}$ in excitation and emission. The UV-Vis spectra were recorded using the same solutions in an Agilent 8453 spectrophotometer.

For that, a series of diluted solutions with absorbance below 0.1 , of quinine sulphate and the desired compound were prepared and the UV-Vis absorption and fluorescence spectra were recorded. To compare both, standard and compound, the integrated emission intensity was plotted versus the absorbance value at $360 \mathrm{~nm}$.

The quantum yield determination was done according to the following steps:

1. Record the UV-Vis absorbance spectrum of the solvent background for the chosen standard. Note down the absorbance at the excitation wavelength to be used.

2. Record the fluorescence spectrum of the same solution in the $10 \mathrm{~mm}$ fluorescence cuvette. Calculate and note down the integrated fluorescence intensity (the area of the fluorescence spectrum) from the fully corrected fluorescence spectrum.

3. Repeat steps 1 . and 2. for five solutions with increasing concentrations of the chosen standard. (There will be six solutions in all, corresponding to absorbances at the excitation wavelength of $\sim 0 /$ solvent blank, $0.02,0.04,0.06,0.08$ and 0.10 .)

4. Repeat steps 1. to 3. for the remaining samples.

5. Plot a graph of integrated fluorescence intensity vs absorbance for each, compound and standard. The result should be a straight line with gradient $m$, and intercept $=0$. These values must fit a linear behavior which relates to the quantum yield according to $\Phi_{f}^{S}=$ $\left(\frac{m_{S}}{m_{Q S}}\right)\left(\frac{n_{S}^{2}}{n_{Q S}^{2}}\right) \Phi_{f}^{Q S}$. Where $\Phi_{f}^{S}$ is the compound QY; $m$ is the gradient of compound (s) and standard (QS); $\mathrm{n}$ is the refractive index of the used solvents and $\Phi_{f}^{Q S}$ is the standard QY.

All quantum yield determinations were fitted to linear regression with $R>0.99$, ensuring the linearity of the data in the measurements.

The uncertainty is estimated to be around \pm 0.04 to \pm 0.10 for the determined QY values, according to the previously reported in IUPAC technical report.

\section{Solvatochromism}

Due to the great similarity between compounds, solvatochromism experiments were done for derivative $\mathbf{4 e}$, as it is the main core in derivatives $\mathbf{9}$ and $\mathbf{1 4}$. The absorption and fluorescence emission spectra were recorded using water, toluene, THF, acetone, acetonitrile, dichloromethane, DMF and methanol solutions in the concentration range of 8 to $1 \times 10^{-5} \mathrm{M}$. 

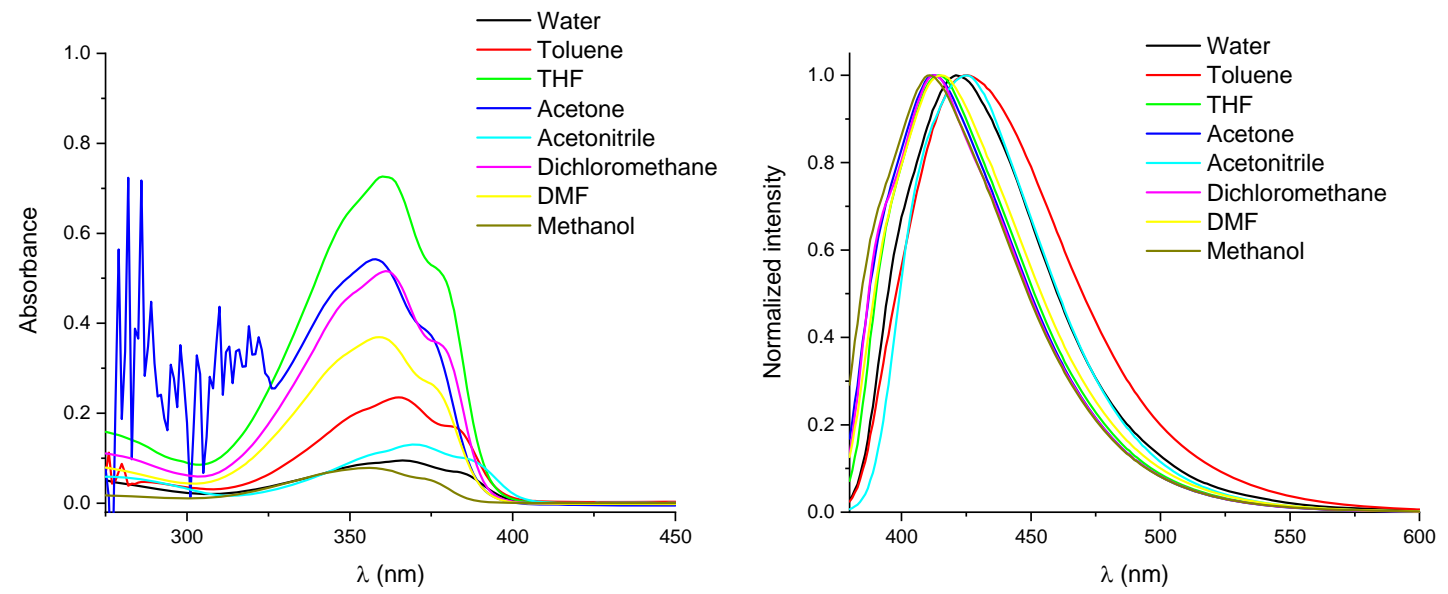

Figure S11. Absorption (left) and emission (right) spectra of $4 \mathbf{e}$ with different solvents.

The same behavior is observed in absorption and emission spectra which are slightly shifted, only observing minor changes.

\section{Triazole click chemistry}

Compound 10, obtained by reaction of benzyl azide with compound $\mathbf{9}$, was studied in terms of fluorescence properties obtaining a $Q Y$ in acetonitrile of $\Phi_{f}=0.94$ and in water $\Phi_{f}=0.67$, which clearly retains a strong fluorescent emission enough to be used as dye in click chemistry. In addition, this derivative also presents a fluorescence lifetime shorter than $5 \mathrm{~ns}, \mathrm{\tau}_{\mathrm{f}}<5 \mathrm{~ns}$.



Figure S12. Optical properties of benzyl triazole derivative $\mathbf{1 0}$ in acetonitrile.

\section{Fluorescence lifetimes}

In all cases the excited state lifetime was recorded using a Horiba Fluorolog-3 spectrofluorometer with a Horiba nanoLED-370 with $1.1 \mathrm{~ns}$ pulse duration and peak wavelength 
at $371 \mathrm{~nm}$. Diluted solutions in acetonitrile or water of $5 \times 10^{-5} \mathrm{M}$ of the different compounds were measured in a $10 \mathrm{~mm}$ quartz cuvette. The fluorescence lifetime was found to be shorter than the LED resolution pulse, which we can conclude that all lifetimes are lower than 5 ns. obtaining in all cases a high-limit value marked by the nano-LED temporal resolution. These compounds present a fluorescence lifetime in all cases, shorter than $5 \mathrm{~ns}, \tau_{\mathrm{f}}<5 \mathrm{~ns}$. 


\section{Cartesian Coordinates}

4a

Computed total energy: $\mathbf{- 1 1 5 6 . 4 2 7 1 5 4 1}$

No imaginary frequencies

$\begin{array}{lrrr}6 & -6.932457 & 3.092131 & 0.337911 \\ 6 & -5.574148 & 3.320970 & 0.396658 \\ 6 & -5.006824 & 4.415401 & -0.294153 \\ 6 & -5.872063 & 5.261937 & -1.043499 \\ 6 & -7.260892 & 4.999856 & -1.082380 \\ 6 & -7.789878 & 3.929697 & -0.401744 \\ 1 & -7.344505 & 2.244486 & 0.877063 \\ 1 & -4.950191 & 2.655857 & 0.975108 \\ 6 & -5.314261 & 6.358540 & -1.741995 \\ 1 & -7.895499 & 5.663584 & -1.662425 \\ 1 & -8.855827 & 3.728603 & -0.431783 \\ 6 & -3.971022 & 6.583255 & -1.686922 \\ 6 & -3.133125 & 5.726312 & -0.932284 \\ 1 & -5.964117 & 7.011421 & -2.317393 \\ 1 & -3.512929 & 7.413882 & -2.210956 \\ 6 & -1.726706 & 6.013035 & -0.907004 \\ 1 & -1.353518 & 6.862368 & -1.464688 \\ 6 & -0.882099 & 5.240534 & -0.195360 \\ 7 & -3.640907 & 4.671748 & -0.253065 \\ 7 & -1.279357 & 4.159267 & 0.529082 \\ 5 & -2.727929 & 3.679240 & 0.621037 \\ 9 & -2.849949 & 2.391980 & 0.098941 \\ 9 & -3.170664 & 3.719661 & 1.942558 \\ 6 & 0.595527 & 5.381831 & -0.037067 \\ 6 & -0.178209 & 3.584777 & 1.146288 \\ 7 & 0.943782 & 4.343989 & 0.789151 \\ 8 & 1.332555 & 6.218189 & -0.526499 \\ 6 & 2.289280 & 4.063363 & 1.257500 \\ 6 & 2.623498 & 4.787420 & 2.557422 \\ 1 & 2.973664 & 4.363764 & 0.458465 \\ 1 & 2.366650 & 2.980002 & 1.384973 \\ 6 & 4.043255 & 4.480678 & 3.016122 \\ 1 & 2.500305 & 5.866406 & 2.406976 \\ 1 & 1.903923 & 4.486580 & 3.327915 \\ 1 & 4.273333 & 5.003843 & 3.948734 \\ 1 & 4.180585 & 3.408074 & 3.192284 \\ 1 & 4.778724 & 4.794275 & 2.267006 \\ 8 & -0.160020 & 2.604691 & 1.861601\end{array}$

A

Computed total energy: $\mathbf{- 1 2 7 1 . 6 6 9 1 4 1 8}$

No imaginary frequencies

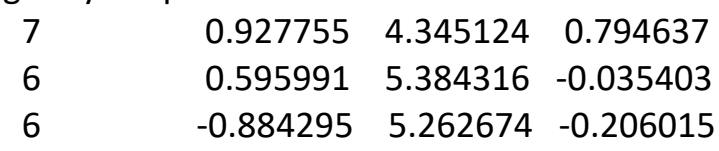




\begin{tabular}{lrrr}
7 & -1.297769 & 4.182811 & 0.522306 \\
6 & -0.208142 & 3.599192 & 1.145617 \\
6 & -1.709847 & 6.037333 & -0.922914 \\
6 & -3.126196 & 5.762936 & -0.960646 \\
6 & -3.943385 & 6.622381 & -1.726900 \\
6 & -5.287546 & 6.406967 & -1.793254 \\
6 & -5.859891 & 5.322992 & -1.096479 \\
6 & -5.018596 & 4.469804 & -0.332033 \\
7 & -3.644970 & 4.716887 & -0.280352 \\
6 & -7.247873 & 5.065430 & -1.142112 \\
6 & -7.803125 & 4.011419 & -0.466816 \\
6 & -6.958780 & 3.167358 & 0.290669 \\
6 & -5.594809 & 3.392395 & 0.356351 \\
5 & -2.749073 & 3.723712 & 0.604199 \\
9 & -3.204850 & 3.771580 & 1.924050 \\
8 & 1.345540 & 6.210873 & -0.520632 \\
8 & -0.202660 & 2.621132 & 1.863819 \\
6 & 2.272474 & 4.044377 & 1.267378 \\
6 & 2.611066 & 4.757696 & 2.573638 \\
6 & 4.026471 & 4.428814 & 3.039012 \\
9 & -2.885121 & 2.430066 & 0.094040 \\
8 & -7.409576 & 2.104065 & 0.989856 \\
1 & -5.000434 & 2.715910 & 0.949343 \\
1 & -7.876637 & 5.725781 & -1.729999 \\
1 & -8.869161 & 3.836453 & -0.519192 \\
1 & -5.925921 & 7.061027 & -2.378323 \\
1 & -3.473619 & 7.445035 & -2.250192 \\
1 & -1.326063 & 6.880377 & -1.480840 \\
1 & 2.961953 & 4.340588 & 0.473338 \\
1 & 2.335516 & 2.961046 & 1.388949 \\
1 & 2.504275 & 5.837928 & 2.428259 \\
1 & 1.885077 & 4.462966 & 3.338737 \\
1 & 4.258584 & 4.944166 & 3.974467 \\
1 & 4.147106 & 3.354338 & 3.210404 \\
1 & 4.768848 & 4.735416 & 2.295156 \\
6 & -8.802873 & 1.799037 & 0.977286 \\
1 & -8.914907 & 0.916270 & 1.603948 \\
1 & -9.388554 & 2.621374 & 1.397485 \\
1 & -9.146162 & 1.572917 & -0.036102 \\
& & & \\
\hline & & &
\end{tabular}

B

Computed total energy: $\mathbf{- 1 3 6 1 . 6 2 7 0 4 0 2}$

No imaginary frequencies

$\begin{array}{lrrr}7 & 0.940279 & 4.346382 & 0.796341 \\ 6 & 0.592498 & 5.382004 & -0.031718 \\ 6 & -0.890278 & 5.245545 & -0.193044 \\ 7 & -1.286862 & 4.164957 & 0.535181 \\ 6 & -0.183763 & 3.590067 & 1.154422 \\ 6 & -1.726887 & 6.016502 & -0.906034 \\ 6 & -3.135327 & 5.727232 & -0.930587 \\ 6 & -3.978198 & 6.583353 & -1.690039 \\ 6 & -5.314000 & 6.359674 & -1.747276\end{array}$




$\begin{array}{llll}6 & -5.872098 & 5.258665 & -1.044047 \\ 6 & -5.009592 & 4.423392 & -0.297353 \\ 7 & -3.639639 & 4.678968 & -0.252294 \\ 6 & -7.259776 & 4.994655 & -1.084713 \\ 6 & -7.795700 & 3.931846 & -0.408148 \\ 6 & -6.917435 & 3.119419 & 0.323287 \\ 6 & -5.564848 & 3.329503 & 0.397899 \\ 5 & -2.728902 & 3.686497 & 0.628407 \\ 9 & -3.186947 & 3.729942 & 1.944889 \\ 8 & 1.326826 & 6.216401 & -0.522991 \\ 8 & -0.171225 & 2.613268 & 1.871056 \\ 6 & 2.292142 & 4.060390 & 1.260527 \\ 6 & 2.634763 & 4.787395 & 2.557998 \\ 6 & 4.057765 & 4.476365 & 3.012368 \\ 9 & -2.865429 & 2.398250 & 0.111673 \\ 7 & -7.473690 & 1.972471 & 1.056128 \\ 1 & -4.957883 & 2.658446 & 0.982670 \\ 1 & -7.897033 & 5.652243 & -1.665006 \\ 1 & -8.854681 & 3.714686 & -0.428309 \\ 1 & -5.964295 & 7.008806 & -2.323459 \\ 1 & -3.520358 & 7.412480 & -2.214161 \\ 1 & -1.353867 & 6.863210 & -1.465260 \\ 1 & 2.972021 & 4.355727 & 0.458035 \\ 1 & 2.364533 & 2.978589 & 1.389608 \\ 1 & 2.515891 & 5.865305 & 2.405262 \\ 1 & 1.918968 & 4.491281 & 3.332092 \\ 1 & 4.292651 & 5.000553 & 3.942156 \\ 1 & 4.191218 & 3.404446 & 3.189877 \\ 1 & 4.789992 & 4.785831 & 2.259729 \\ 8 & -8.681877 & 1.788407 & 0.991643 \\ 8 & -6.707562 & 1.261218 & 1.691439\end{array}$

\section{C}

Computed total energy: $\mathbf{- 1 2 7 1 . 6 6 9 3 0 2 9}$

No imaginary frequencies

$\begin{array}{llll}7 & 0.961465 & 4.346737 & 0.799205 \\ 6 & 0.630675 & 5.386012 & -0.031897 \\ 6 & -0.848264 & 5.264539 & -0.204551 \\ 7 & -1.263116 & 4.183193 & 0.523910 \\ 6 & -0.174348 & 3.600516 & 1.148816 \\ 6 & -1.674627 & 6.037706 & -0.921304 \\ 6 & -3.090724 & 5.760143 & -0.957900 \\ 6 & -3.917649 & 6.615015 & -1.723000 \\ 6 & -5.258703 & 6.397360 & -1.788154 \\ 6 & -5.832354 & 5.308557 & -1.088189 \\ 6 & -4.979395 & 4.470347 & -0.331954 \\ 7 & -3.610769 & 4.717038 & -0.280268 \\ 6 & -7.225810 & 5.065611 & -1.145329 \\ 6 & -7.763709 & 4.003155 & -0.459780 \\ 6 & -6.912441 & 3.161813 & 0.296847 \\ 6 & -5.564748 & 3.381667 & 0.363366 \\ 5 & -2.712186 & 3.722634 & 0.606684\end{array}$




$\begin{array}{lrrr}9 & -3.172696 & 3.772572 & 1.924455 \\ 8 & 1.381735 & 6.212505 & -0.515360 \\ 8 & -0.169531 & 2.622296 & 1.867284 \\ 6 & 2.305381 & 4.047316 & 1.274855 \\ 6 & 2.638393 & 4.756611 & 2.584744 \\ 6 & 4.052548 & 4.428136 & 3.054182 \\ 9 & -2.852103 & 2.430354 & 0.095197 \\ 1 & -7.356568 & 2.328400 & 0.829987 \\ 1 & -4.954800 & 2.715186 & 0.951946 \\ 1 & -7.836487 & 5.735034 & -1.737269 \\ 8 & -9.073254 & 3.670253 & -0.436415 \\ 1 & -5.897468 & 7.050646 & -2.373261 \\ 1 & -3.453267 & 7.439868 & -2.248333 \\ 1 & -1.292880 & 6.881436 & -1.479724 \\ 1 & 2.996380 & 4.348348 & 0.483965 \\ 1 & 2.370984 & 2.963680 & 1.392557 \\ 1 & 2.530597 & 5.837187 & 2.442603 \\ 1 & 1.910106 & 4.458325 & 3.346265 \\ 1 & 4.280777 & 4.941149 & 3.991878 \\ 1 & 4.173848 & 3.353327 & 3.223027 \\ 1 & 4.797189 & 4.737658 & 2.313806 \\ 6 & -9.986275 & 4.475343 & -1.176177 \\ 1 & -10.968901 & 4.035149 & -1.017589 \\ 1 & -9.981732 & 5.506396 & -0.809946 \\ 1 & -9.743942 & 4.459079 & -2.243040\end{array}$

D

Computed total energy: $\mathbf{- 1 3 6 1 . 6 2 7 9 7 2}$

No imaginary frequencies

$\begin{array}{lrrr}7 & 0.938466 & 4.349524 & 0.795708 \\ 6 & 0.588620 & 5.386330 & -0.029828 \\ 6 & -0.894426 & 5.247569 & -0.190358 \\ 7 & -1.288714 & 4.165971 & 0.535594 \\ 6 & -0.184450 & 3.591075 & 1.153029 \\ 6 & -1.732378 & 6.018621 & -0.902365 \\ 6 & -3.139867 & 5.729375 & -0.929717 \\ 6 & -3.978012 & 6.585244 & -1.689681 \\ 6 & -5.314047 & 6.359043 & -1.749760 \\ 6 & -5.869142 & 5.257692 & -1.047748 \\ 6 & -5.012114 & 4.417759 & -0.295435 \\ 7 & -3.645828 & 4.676957 & -0.250651 \\ 6 & -7.252759 & 4.998197 & -1.095586 \\ 6 & -7.750951 & 3.926987 & -0.405825 \\ 6 & -6.923506 & 3.079650 & 0.346887 \\ 6 & -5.575091 & 3.321214 & 0.400915 \\ 5 & -2.731167 & 3.685606 & 0.629151 \\ 9 & -3.183689 & 3.728891 & 1.947105 \\ 8 & 1.320902 & 6.222950 & -0.520151 \\ 8 & -0.170090 & 2.612915 & 1.867669 \\ 6 & 2.291147 & 4.063443 & 1.257520 \\ 6 & 2.633565 & 4.784037 & 2.558602 \\ 6 & 4.057012 & 4.471797 & 3.010743\end{array}$




$\begin{array}{lrrr}9 & -2.861263 & 2.397710 & 0.111103 \\ 1 & -7.353523 & 2.241747 & 0.879285 \\ 1 & -4.951139 & 2.662308 & 0.982425 \\ 1 & -7.906111 & 5.641251 & -1.671488 \\ 7 & -9.187656 & 3.656492 & -0.454783 \\ 1 & -5.964971 & 7.006671 & -2.326869 \\ 1 & -3.519743 & 7.414923 & -2.212186 \\ 1 & -1.359553 & 6.866476 & -1.460000 \\ 1 & 2.969855 & 4.364245 & 0.456078 \\ 1 & 2.365585 & 2.981091 & 1.380759 \\ 1 & 2.513677 & 5.862619 & 2.411510 \\ 1 & 1.918362 & 4.483267 & 3.331443 \\ 1 & 4.291927 & 4.991437 & 3.943069 \\ 1 & 4.191337 & 3.399086 & 3.182746 \\ 1 & 4.788672 & 4.785605 & 2.259356 \\ 8 & -9.894249 & 4.400407 & -1.123116 \\ 8 & -9.611707 & 2.697069 & 0.176769\end{array}$

\section{E}

Computed total energy: $\mathbf{- 1 2 7 1 . 6 7 5 2 7 4 7}$

No imaginary frequencies

$\begin{array}{lrrr}7 & 0.966500 & 4.330307 & 0.817176 \\ 6 & 0.627538 & 5.367439 & -0.012190 \\ 6 & -0.852612 & 5.238505 & -0.178650 \\ 7 & -1.258660 & 4.156084 & 0.551773 \\ 6 & -0.165333 & 3.578676 & 1.171694 \\ 6 & -1.681903 & 6.008964 & -0.893930 \\ 6 & -3.099787 & 5.726964 & -0.929024 \\ 6 & -3.908358 & 6.584021 & -1.694599 \\ 6 & -5.262826 & 6.371679 & -1.768384 \\ 6 & -5.835151 & 5.268572 & -1.057841 \\ 6 & -4.981426 & 4.434030 & -0.301925 \\ 7 & -3.606656 & 4.678872 & -0.245776 \\ 6 & -7.222051 & 5.013750 & -1.108420 \\ 6 & -7.759498 & 3.952288 & -0.424512 \\ 6 & -6.913694 & 3.119057 & 0.328372 \\ 6 & -5.558740 & 3.347515 & 0.392960 \\ 5 & -2.708461 & 3.691997 & 0.635782 \\ 9 & -3.160560 & 3.735980 & 1.958508 \\ 8 & 1.371989 & 6.197387 & -0.499893 \\ 8 & -0.151684 & 2.600844 & 1.890616 \\ 6 & 2.314019 & 4.035558 & 1.285306 \\ 6 & 2.655401 & 4.752679 & 2.588758 \\ 6 & 4.073963 & 4.429922 & 3.048803 \\ 9 & -2.838705 & 2.395000 & 0.128798 \\ 1 & -7.331030 & 2.277773 & 0.871360 \\ 1 & -4.939540 & 2.688432 & 0.979959 \\ 1 & -7.847191 & 5.672860 & -1.697488 \\ 1 & -8.825173 & 3.756675 & -0.463554 \\ 8 & -6.110987 & 7.121997 & -2.461709 \\ 1 & -3.427472 & 7.400968 & -2.212083 \\ 1 & -1.301585 & 6.853214 & -1.452324\end{array}$




$\begin{array}{llll}1 & 2.999509 & 4.332518 & 0.488076 \\ 1 & 2.381416 & 2.952720 & 1.408985 \\ 1 & 2.544039 & 5.832236 & 2.441775 \\ 1 & 1.933701 & 4.456763 & 3.357458 \\ 1 & 4.308121 & 4.947748 & 3.982382 \\ 1 & 4.199326 & 3.356195 & 3.221519 \\ 1 & 4.812072 & 4.737959 & 2.301289 \\ 6 & -5.613310 & 8.243646 & -3.201116 \\ 1 & -6.485306 & 8.689517 & -3.673664 \\ 1 & -5.143636 & 8.965113 & -2.528779 \\ 1 & -4.904188 & 7.913111 & -3.963453\end{array}$

\section{$\mathbf{F}$}

Computed total energy: $\mathbf{- 1 3 6 1 . 6 1 2 1 0 2 8}$

No imaginary frequencies

$\begin{array}{lrrr}7 & 0.936641 & 4.347157 & 0.782911 \\ 6 & 0.586543 & 5.387559 & -0.038097 \\ 6 & -0.896873 & 5.250085 & -0.196970 \\ 7 & -1.291401 & 4.166087 & 0.522904 \\ 6 & -0.185859 & 3.588362 & 1.138712 \\ 6 & -1.737037 & 6.024198 & -0.903361 \\ 6 & -3.141945 & 5.727347 & -0.925030 \\ 6 & -3.980190 & 6.581327 & -1.686724 \\ 6 & -5.304045 & 6.320322 & -1.752453 \\ 6 & -5.905900 & 5.239306 & -1.057160 \\ 6 & -5.027522 & 4.423010 & -0.292674 \\ 7 & -3.656062 & 4.681903 & -0.253001 \\ 6 & -7.294898 & 4.973788 & -1.059869 \\ 6 & -7.799573 & 3.922452 & -0.340409 \\ 6 & -6.930395 & 3.102171 & 0.400963 \\ 6 & -5.578311 & 3.341636 & 0.430046 \\ 5 & -2.734748 & 3.689387 & 0.630915 \\ 9 & -3.174123 & 3.748256 & 1.951063 \\ 8 & 1.317870 & 6.226101 & -0.525804 \\ 8 & -0.172852 & 2.608536 & 1.850363 \\ 6 & 2.289466 & 4.060126 & 1.244320 \\ 6 & 2.629483 & 4.774554 & 2.549443 \\ 6 & 4.052790 & 4.461855 & 3.001668 \\ 9 & -2.871597 & 2.401595 & 0.120497 \\ 1 & -7.329511 & 2.266887 & 0.965629 \\ 1 & -4.941394 & 2.699113 & 1.015417 \\ 1 & -7.956708 & 5.602834 & -1.639107 \\ 1 & -8.865086 & 3.723964 & -0.344156 \\ 7 & -6.108192 & 7.217406 & -2.601134 \\ 1 & -3.542861 & 7.419856 & -2.211701 \\ 1 & -1.368398 & 6.874579 & -1.459680 \\ 1 & 2.968259 & 4.366034 & 0.444929 \\ 1 & 2.365135 & 2.977236 & 1.361837 \\ 1 & 2.508381 & 5.853701 & 2.407649 \\ 1 & 1.913815 & 4.468974 & 3.319953 \\ 1 & 4.285885 & 4.977345 & 3.936746 \\ 1 & 4.188248 & 3.388513 & 3.168719\end{array}$




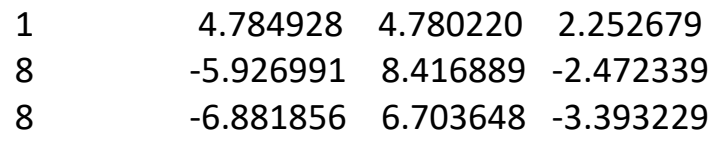

G

Computed total energy: $\mathbf{- 1 2 7 1 . 6 6 5 0 1 3}$

No imaginary frequencies

$\begin{array}{lrrr}7 & 1.118928 & 4.161012 & 1.054060 \\ 6 & 0.845032 & 5.202525 & 0.205073 \\ 6 & -0.630016 & 5.113160 & -0.025642 \\ 7 & -1.096883 & 4.050681 & 0.691082 \\ 6 & -0.046395 & 3.444784 & 1.363023 \\ 6 & -1.404547 & 5.902858 & -0.784409 \\ 6 & -2.819419 & 5.655553 & -0.877138 \\ 6 & -3.599335 & 6.531407 & -1.700968 \\ 6 & -4.942900 & 6.337284 & -1.815103 \\ 6 & -5.559087 & 5.269196 & -1.119533 \\ 6 & -4.771122 & 4.415655 & -0.313563 \\ 7 & -3.397955 & 4.638279 & -0.213719 \\ 6 & -6.953112 & 5.047862 & -1.223406 \\ 6 & -7.547948 & 4.013213 & -0.550011 \\ 6 & -6.760241 & 3.163692 & 0.252374 \\ 6 & -5.404640 & 3.353182 & 0.372960 \\ 5 & -2.559855 & 3.630718 & 0.722607 \\ 9 & -3.067995 & 3.714426 & 2.019699 \\ 8 & 1.632068 & 6.008519 & -0.253371 \\ 8 & -0.096132 & 2.472169 & 2.085973 \\ 6 & 2.435274 & 3.836832 & 1.588139 \\ 6 & 2.726221 & 4.549904 & 2.905901 \\ 6 & 4.111846 & 4.197407 & 3.438752 \\ 9 & -2.712422 & 2.337434 & 0.221587 \\ 1 & -7.227432 & 2.343590 & 0.786728 \\ 1 & -4.830938 & 2.684870 & 0.994726 \\ 1 & -7.536914 & 5.715975 & -1.847906 \\ 1 & -8.616339 & 3.846698 & -0.631988 \\ 1 & -5.557687 & 6.983525 & -2.428604 \\ 8 & -2.894012 & 7.506169 & -2.309038 \\ 1 & -0.981570 & 6.730773 & -1.331726 \\ 1 & 3.165136 & 4.117008 & 0.825008 \\ 1 & 2.472611 & 2.753038 & 1.715953 \\ 1 & 2.646405 & 5.631190 & 2.751365 \\ 1 & 1.959948 & 4.272386 & 3.637393 \\ 1 & 4.309264 & 4.713428 & 4.381774 \\ 1 & 4.204230 & 3.121848 & 3.620552 \\ 1 & 4.893957 & 4.486336 & 2.729369 \\ 6 & -3.595831 & 8.420592 & -3.149239 \\ 1 & -4.346889 & 8.973434 & -2.577793 \\ 1 & -2.842927 & 9.108196 & -3.528638 \\ 1 & -4.069852 & 7.894700 & -3.982905\end{array}$

H

Computed total energy: $\mathbf{- 1 3 6 1 . 6 1 2 0 2 2}$ 
No imaginary frequencies

\begin{tabular}{lrrr}
7 & 0.961596 & 4.339622 & 0.804952 \\
6 & 0.616655 & 5.384164 & -0.013189 \\
6 & -0.862471 & 5.229827 & -0.206188 \\
7 & -1.257357 & 4.138486 & 0.495319 \\
6 & -0.159312 & 3.565469 & 1.129665 \\
6 & -1.681107 & 6.007686 & -0.933709 \\
6 & -3.088716 & 5.722066 & -0.994996 \\
6 & -3.982412 & 6.567702 & -1.718890 \\
6 & -5.329279 & 6.382880 & -1.698944 \\
6 & -5.866745 & 5.285776 & -1.003215 \\
6 & -4.980461 & 4.417686 & -0.318636 \\
7 & -3.607873 & 4.677774 & -0.312426 \\
6 & -7.260560 & 5.044390 & -1.001020 \\
6 & -7.770265 & 3.960302 & -0.337674 \\
6 & -6.889457 & 3.091039 & 0.334687 \\
6 & -5.529840 & 3.303324 & 0.351077 \\
5 & -2.698749 & 3.663545 & 0.564484 \\
9 & -3.162775 & 3.691610 & 1.876484 \\
8 & 1.347490 & 6.235856 & -0.477284 \\
8 & -0.152384 & 2.578835 & 1.831375 \\
6 & 2.307311 & 4.064824 & 1.294251 \\
6 & 2.608350 & 4.772048 & 2.612733 \\
6 & 4.023932 & 4.471511 & 3.096341 \\
9 & -2.828615 & 2.388774 & 0.019320 \\
1 & -7.287616 & 2.227427 & 0.856264 \\
1 & -4.897046 & 2.608918 & 0.878437 \\
1 & -7.906609 & 5.731914 & -1.536030 \\
1 & -8.836689 & 3.767247 & -0.331033 \\
1 & -5.979249 & 7.064196 & -2.235556 \\
7 & -3.497804 & 7.685090 & -2.531587 \\
1 & -1.272680 & 6.849162 & -1.467391 \\
1 & 3.000031 & 4.385797 & 0.512882 \\
1 & 2.393565 & 2.981931 & 1.404198 \\
1 & 2.478300 & 5.850919 & 2.476887 \\
1 & 1.878187 & 4.452023 & 3.363520 \\
1 & 4.229151 & 4.982544 & 4.040350 \\
1 & 4.167174 & 3.398494 & 3.258880 \\
1 & 4.770001 & 4.803376 & 2.367262 \\
8 & -2.462147 & 7.543382 & -3.167455 \\
8 & -4.190122 & 8.692461 & -2.560715 \\
\hline & & &
\end{tabular}




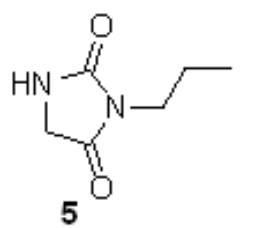

${ }^{1} \mathrm{H}-\mathrm{NMR}\left(500 \mathrm{MHz}_{1} \mathrm{CDCl}_{3}\right)$
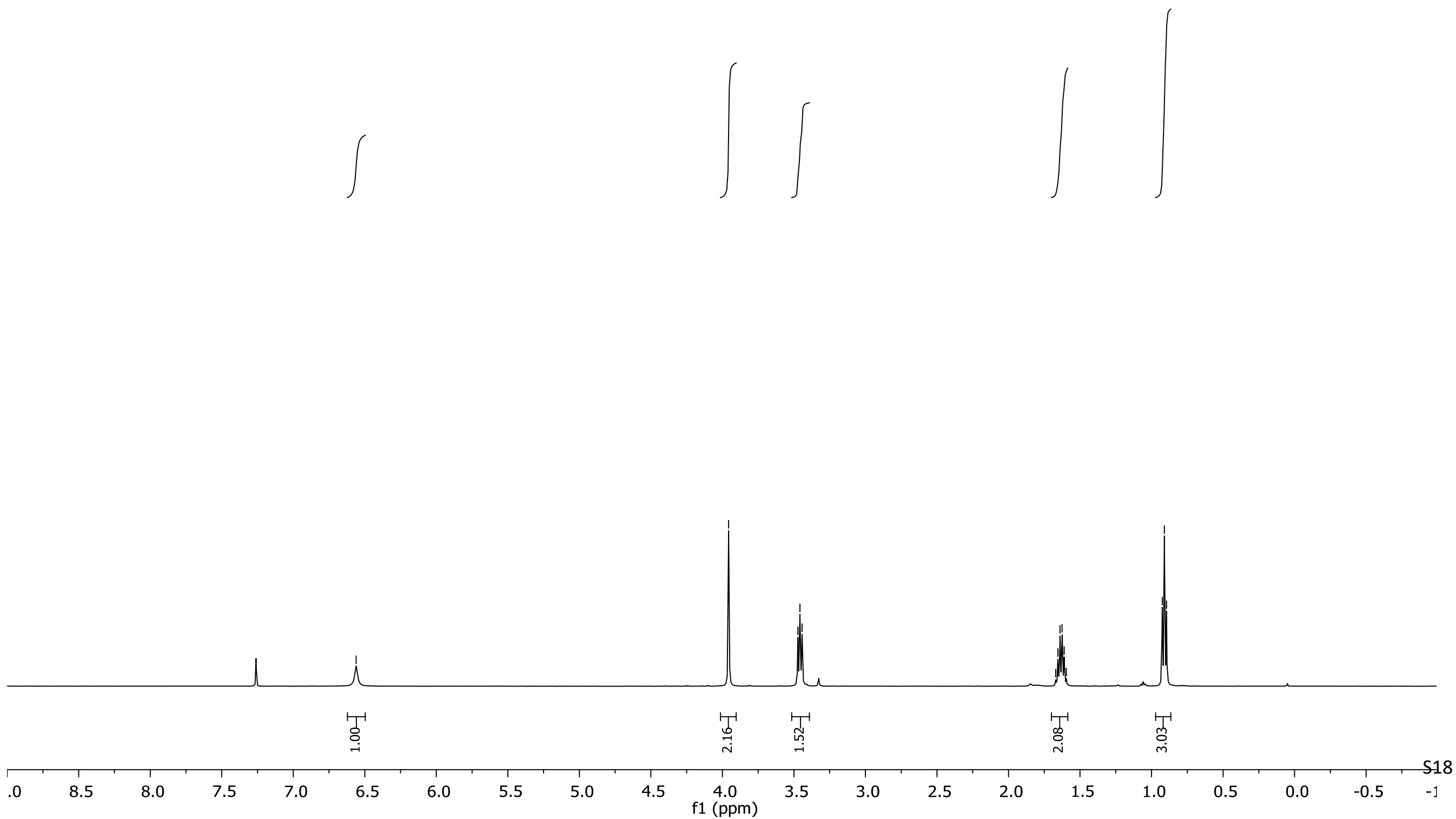


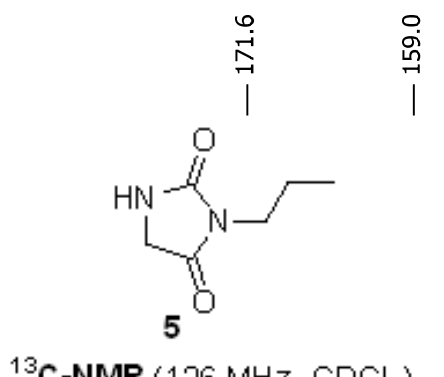

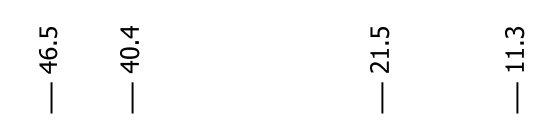

${ }^{13} \mathrm{C}-\mathrm{NMR}\left(126 \mathrm{MHz}, \mathrm{CDCl}_{3}\right)$



$90 \quad 180$

$170 \quad 160$

150

140

130

120

110

100

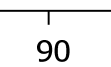

90

80

70

60

50

40

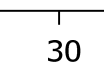




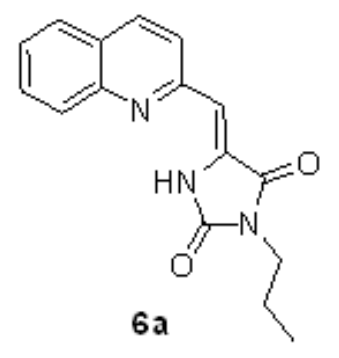

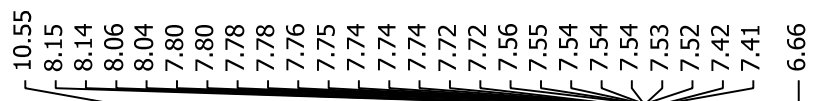

ตำ

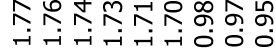

${ }^{1}$ HeNMR $\left(500 \mathrm{MHz}_{1} \mathrm{CDCl}_{3}\right)$
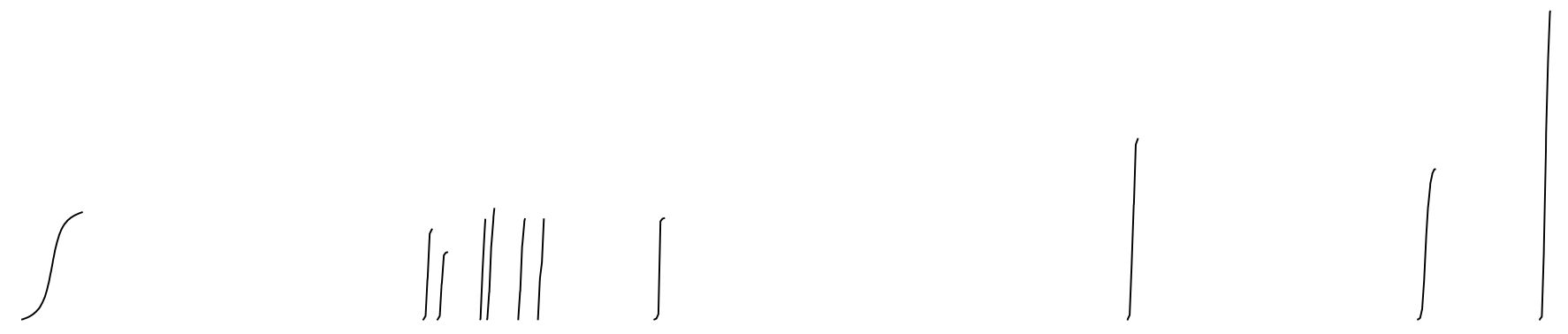

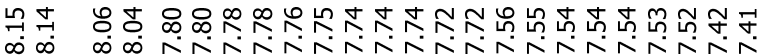

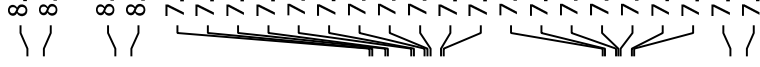

r
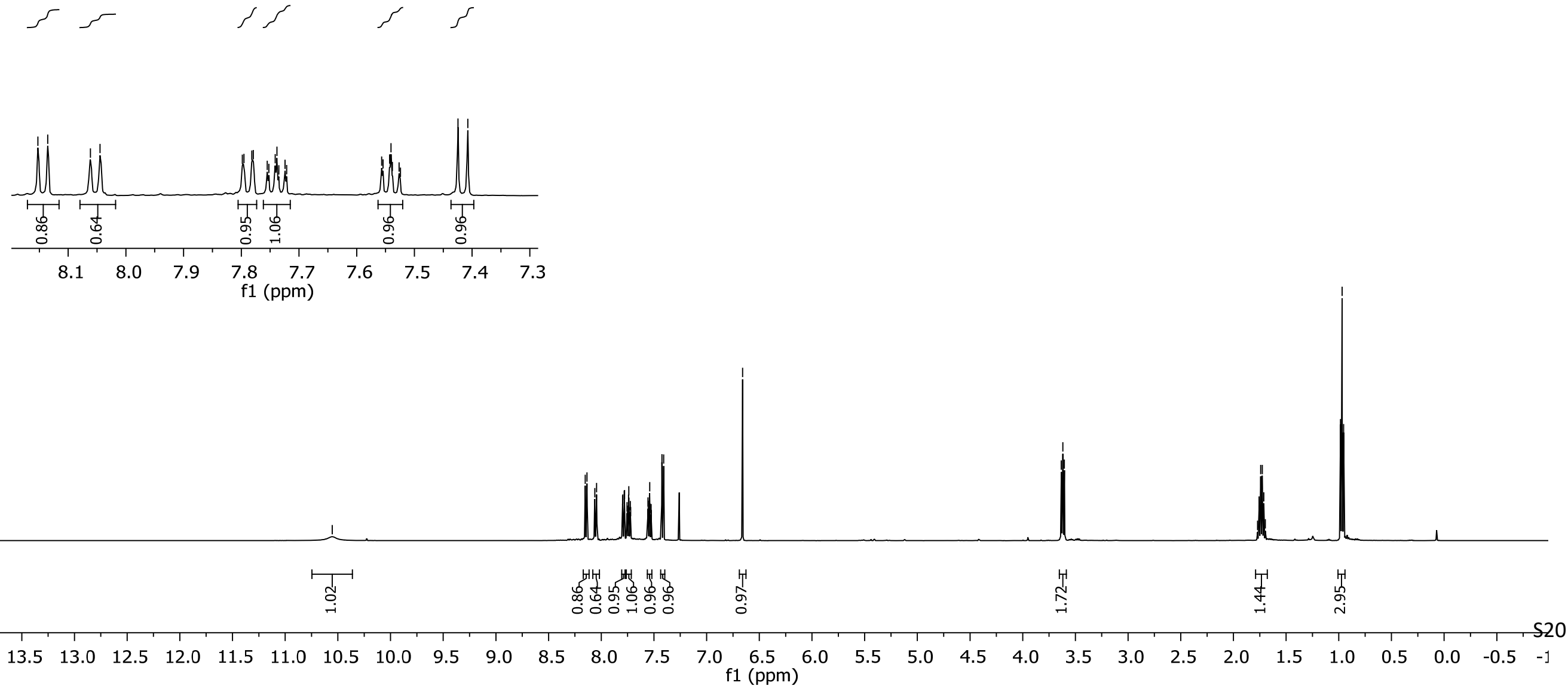


l

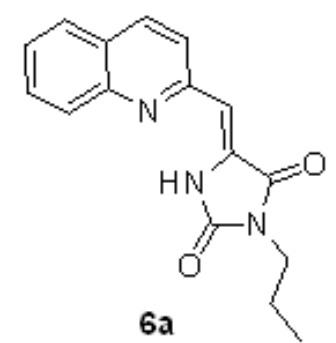

${ }^{13} \mathrm{C}-\mathrm{NMR}\left(126 \mathrm{MHz}_{1} \mathrm{CDCl}_{3}\right)$

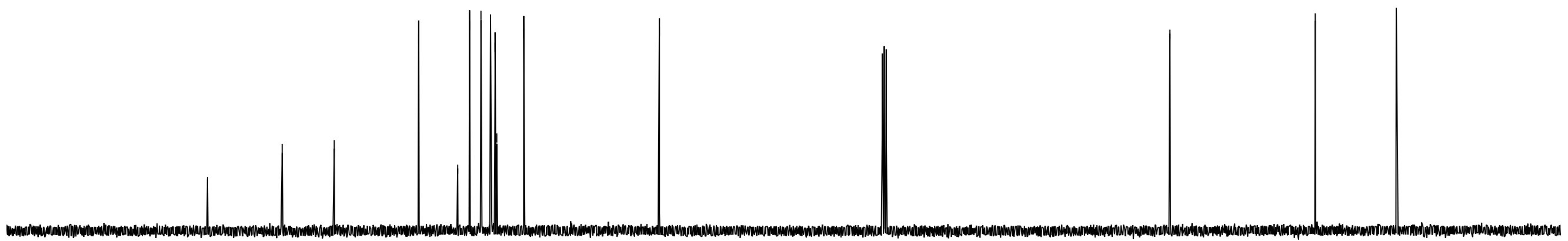

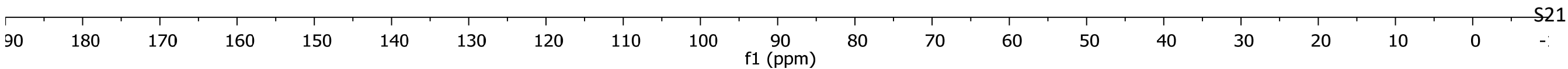






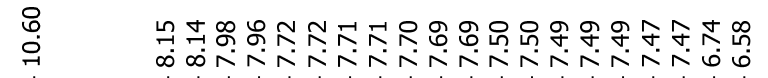

ஜே ஜூ

|



।

V

${ }^{1}$ H-NMR $\left(500 \mathrm{MHz}_{1} \mathrm{CDCl}_{3}\right)$
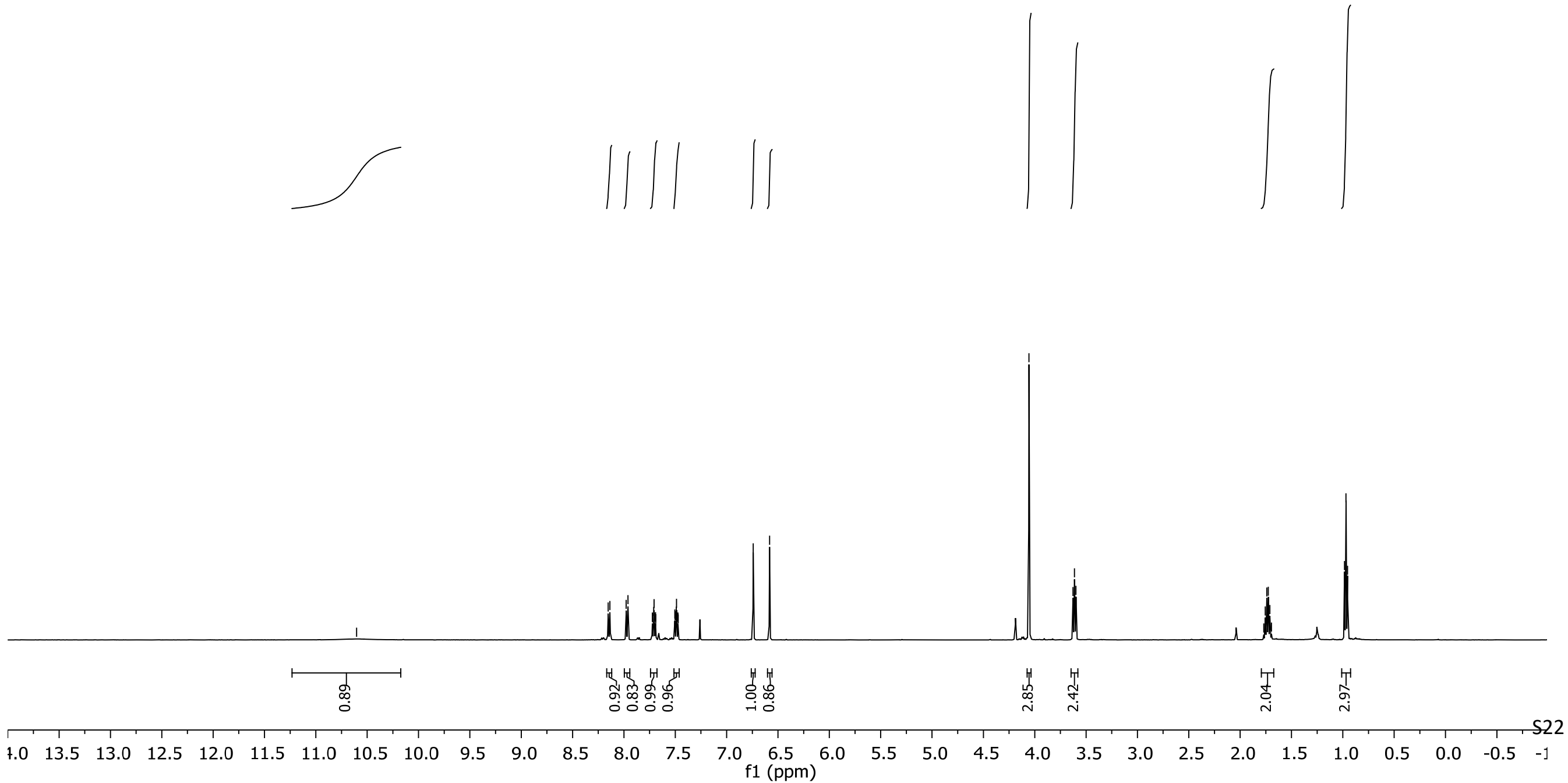


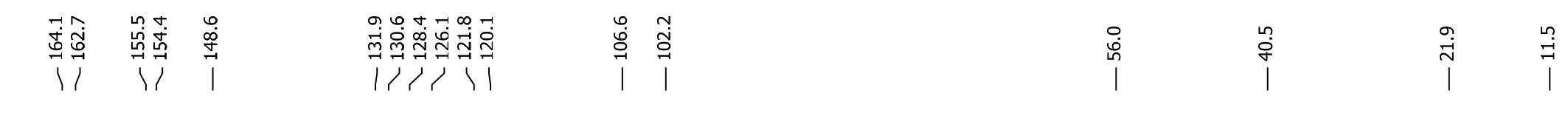

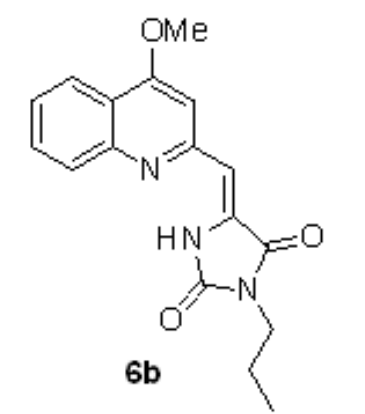

${ }^{13} \mathrm{C}$-NMR $(75 \mathrm{MHZ}, \mathrm{CDC} b)$

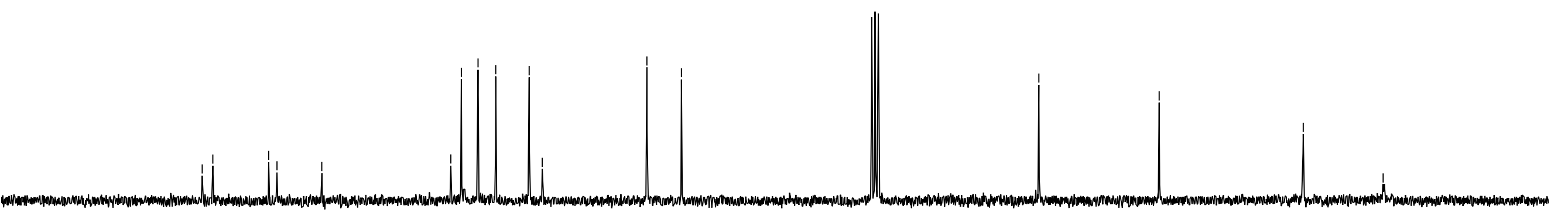

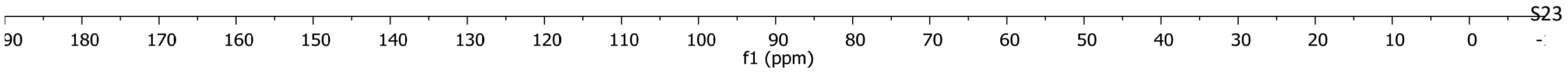




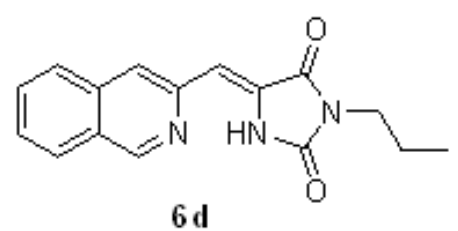

\section{$6 \mathrm{~d}$}

${ }^{1} \mathrm{H}-\mathrm{NMR}\left(500 \mathrm{MHz}, \mathrm{CDCl}_{3}\right)$
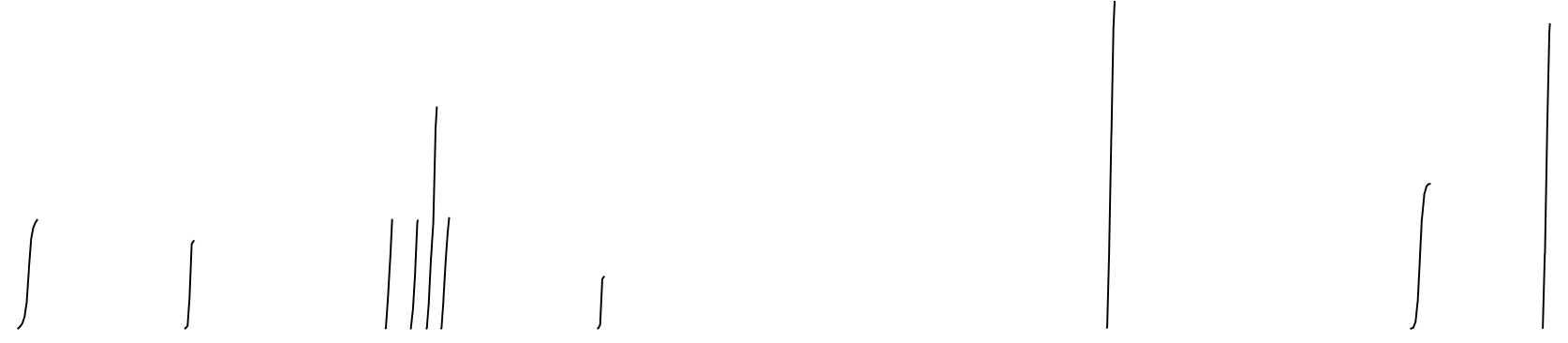

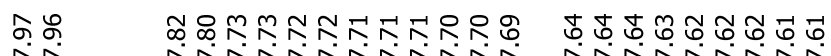

iा

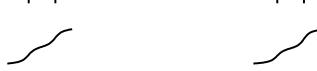
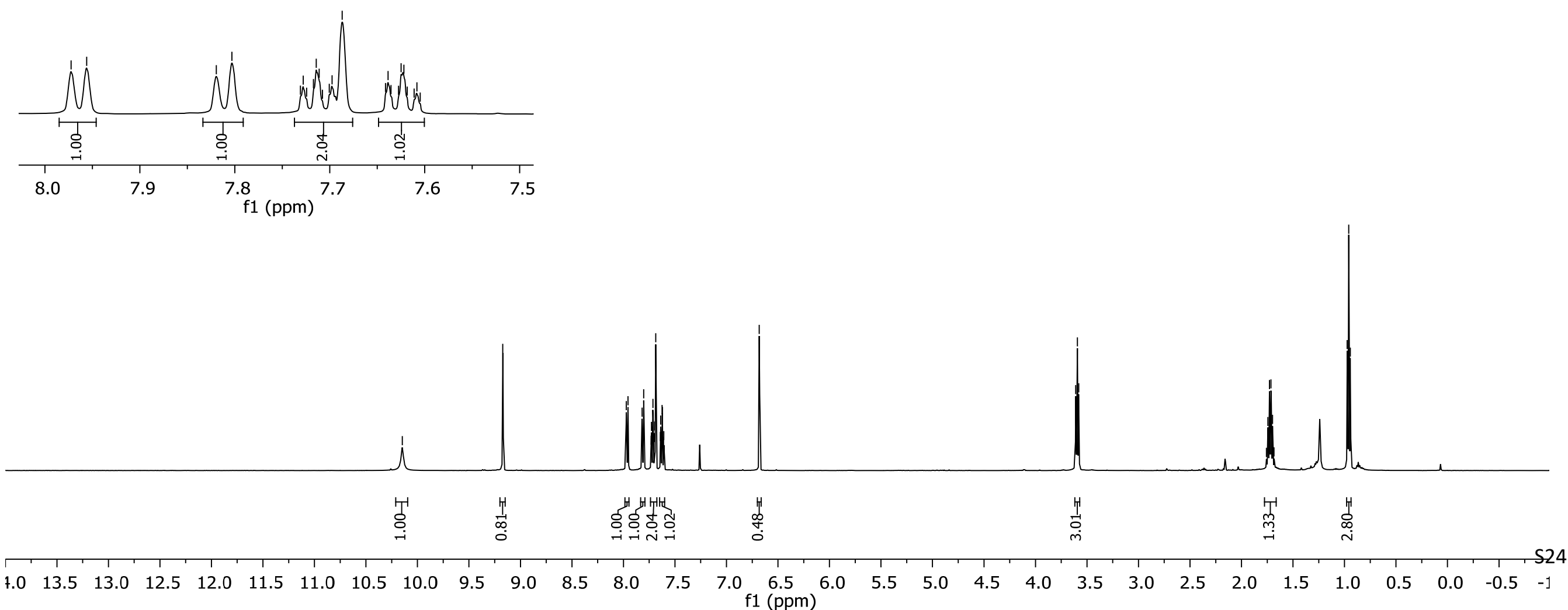

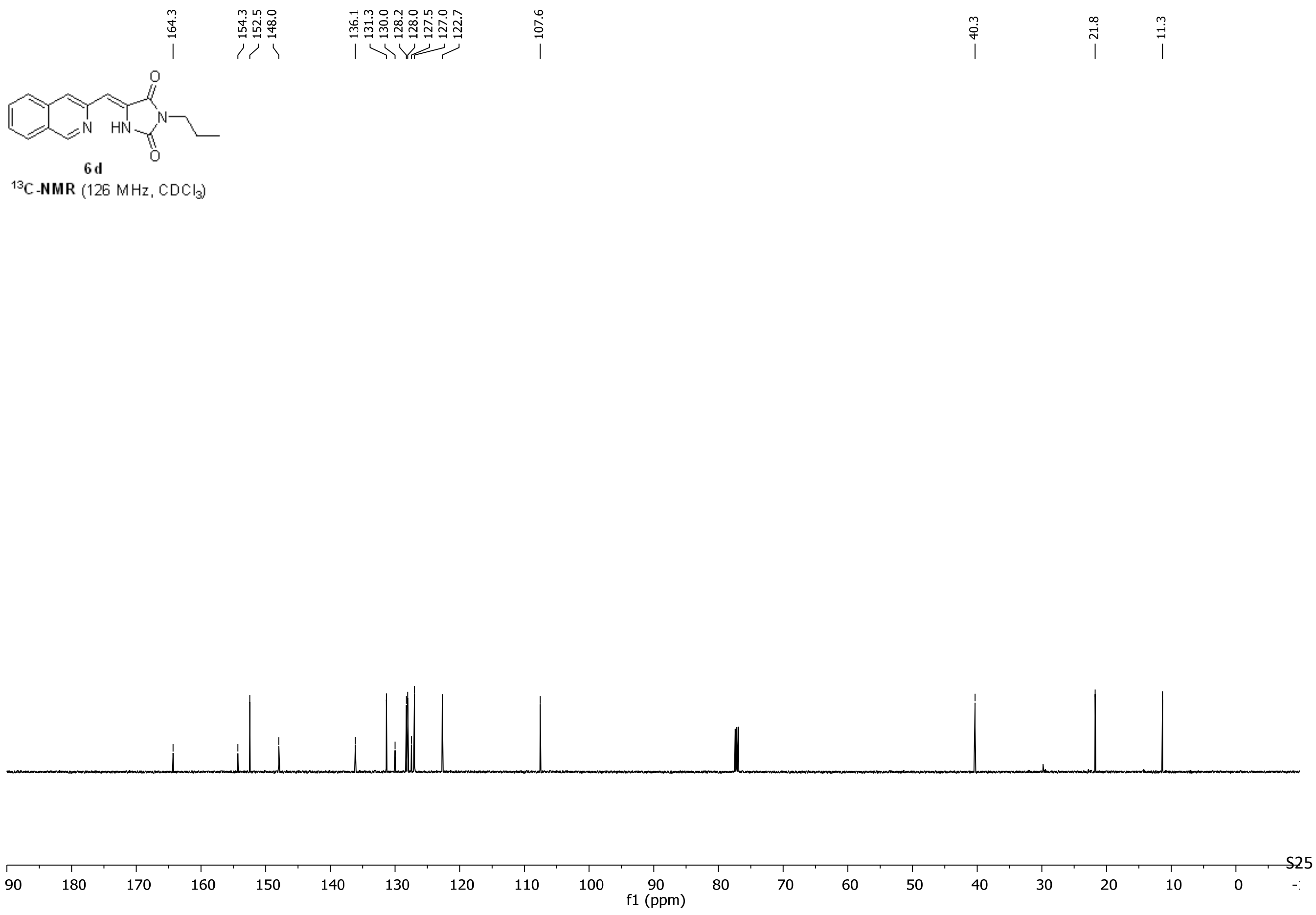

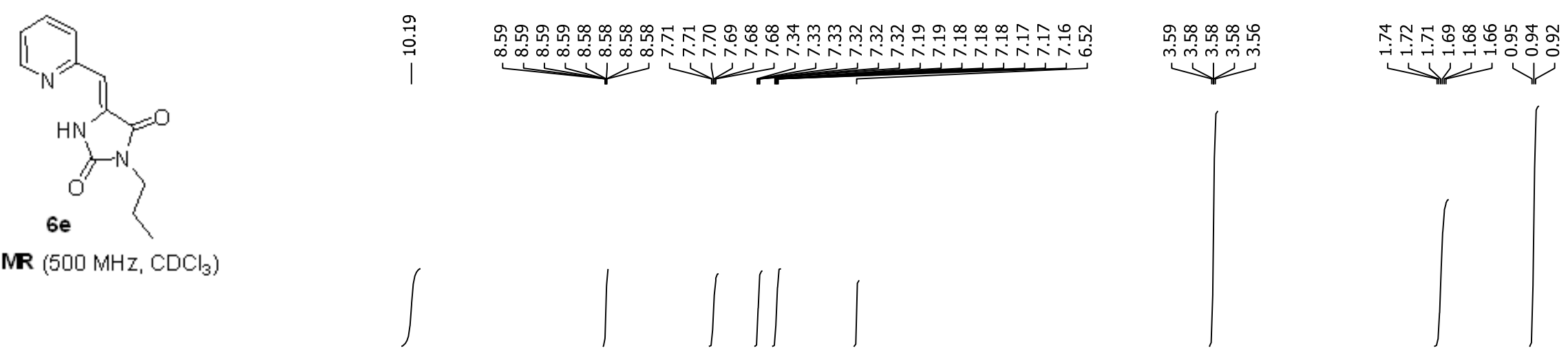

${ }^{1}$ H-NMR $\left(500 \mathrm{MHz}_{1} \mathrm{CDCl}_{3}\right)$
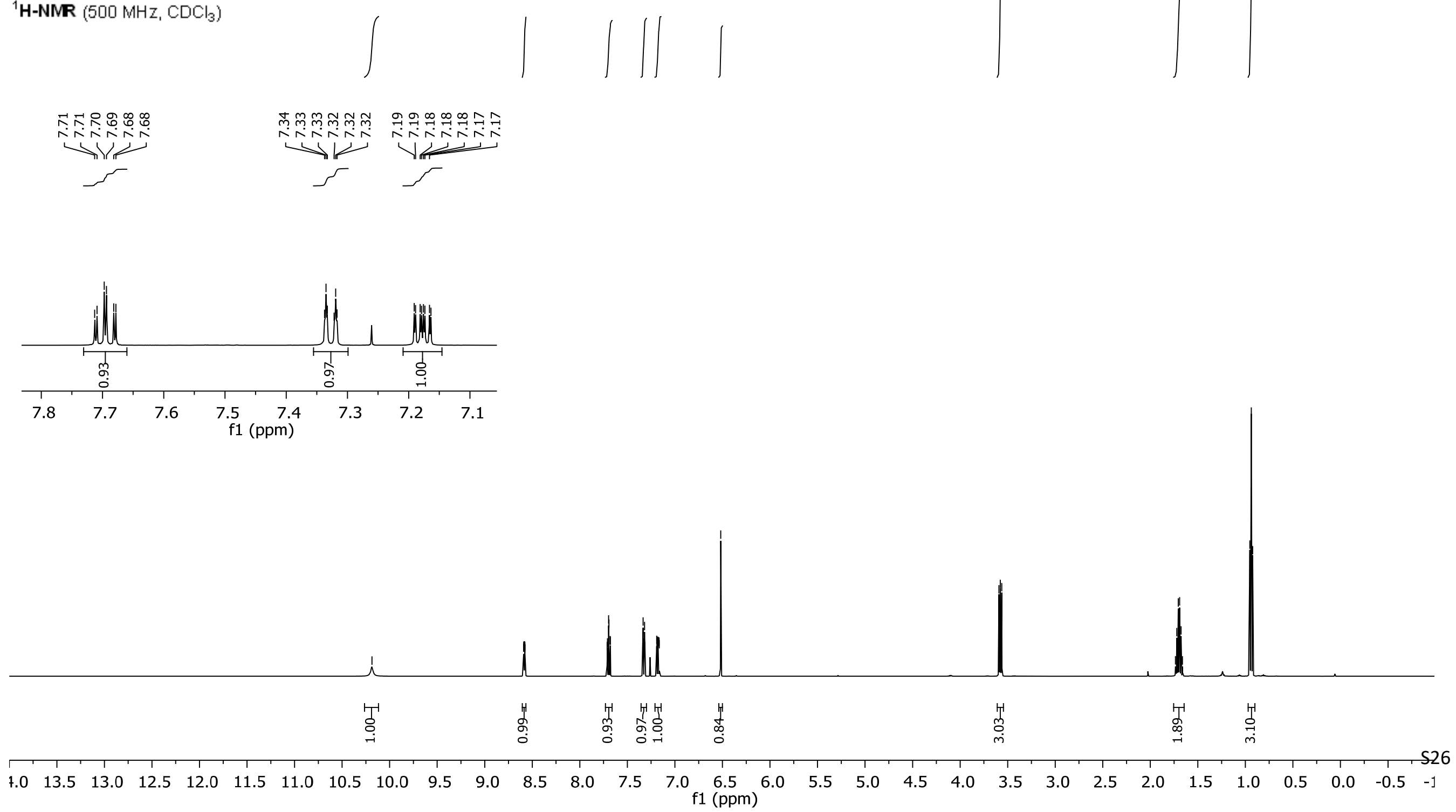

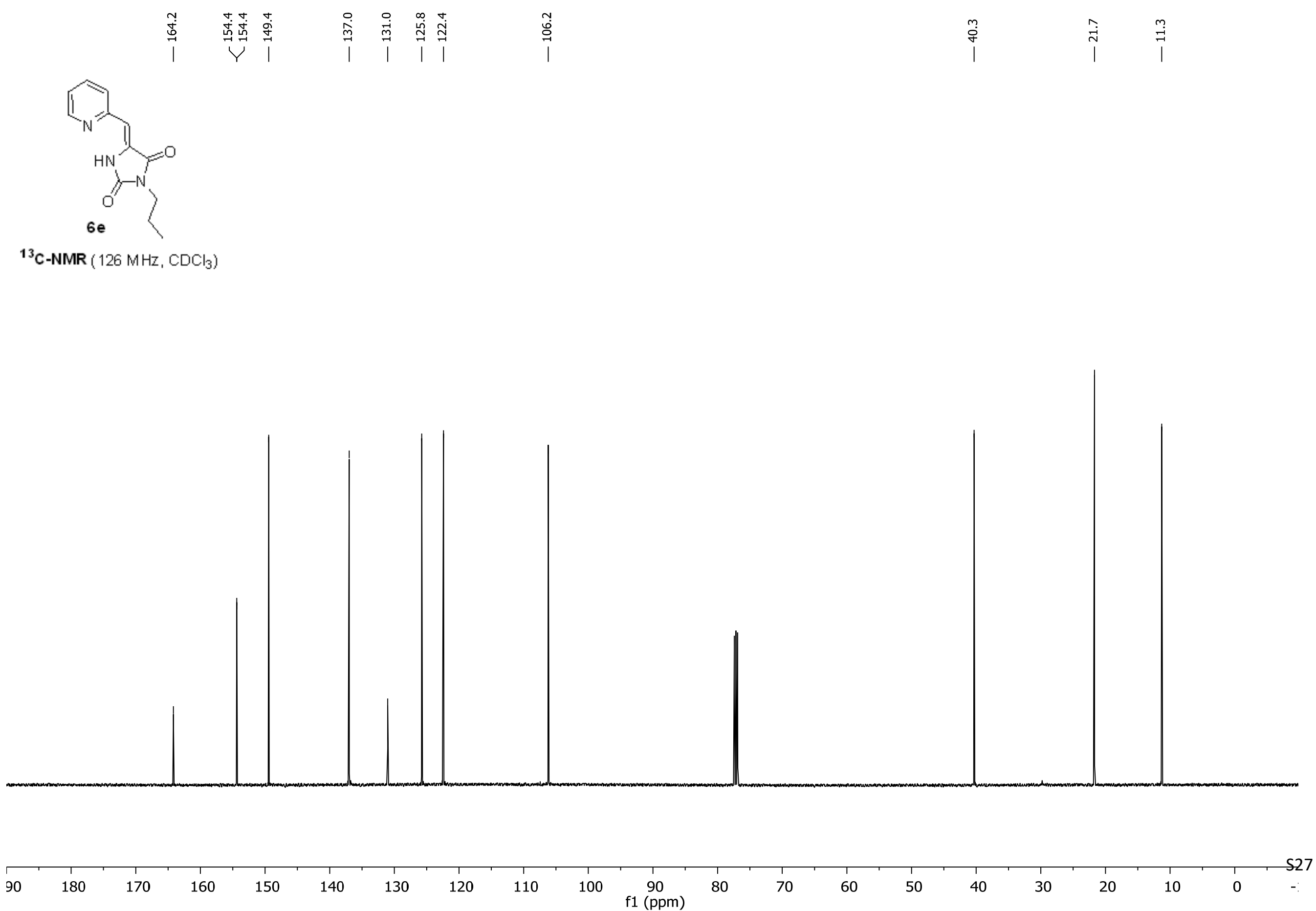


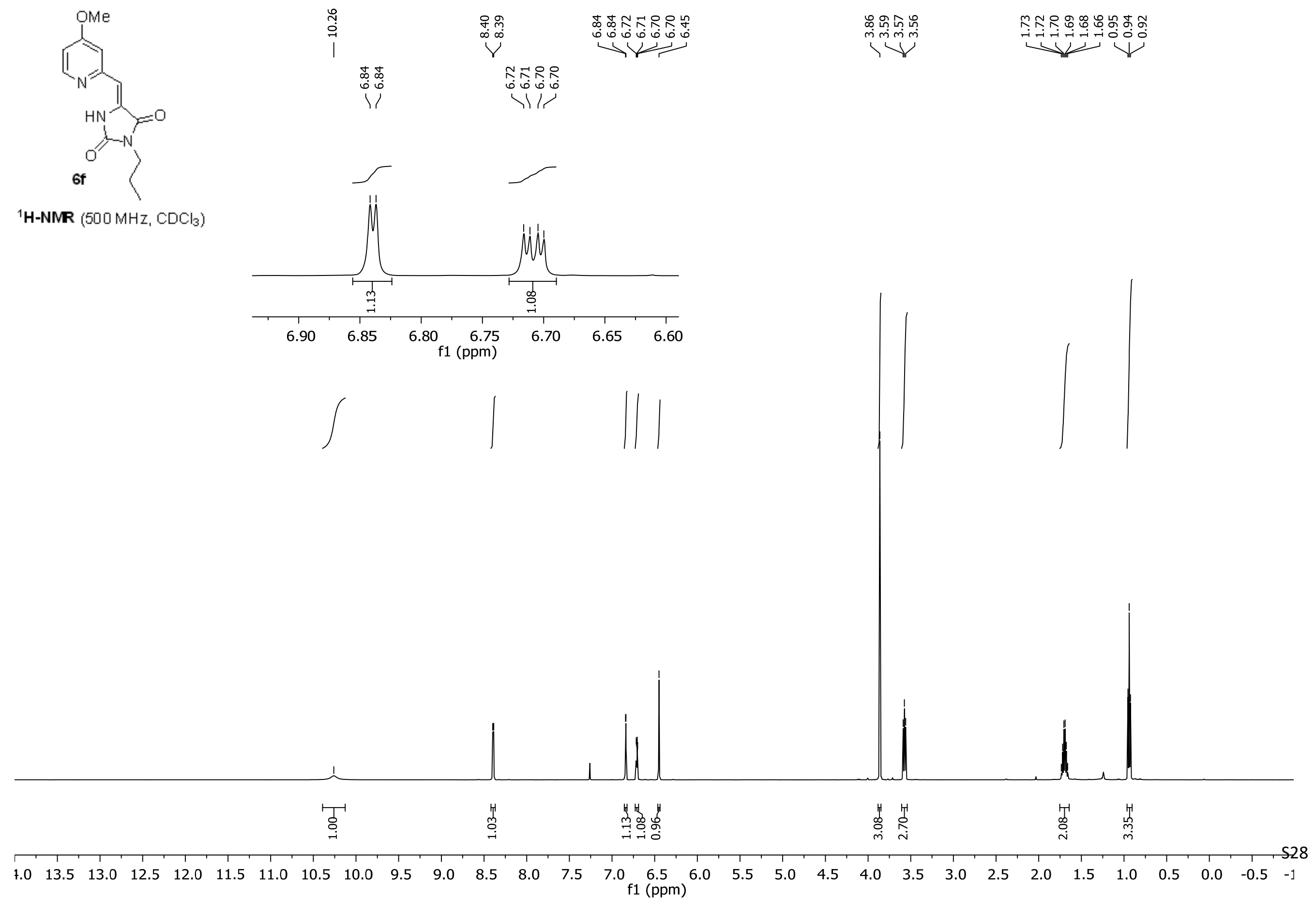



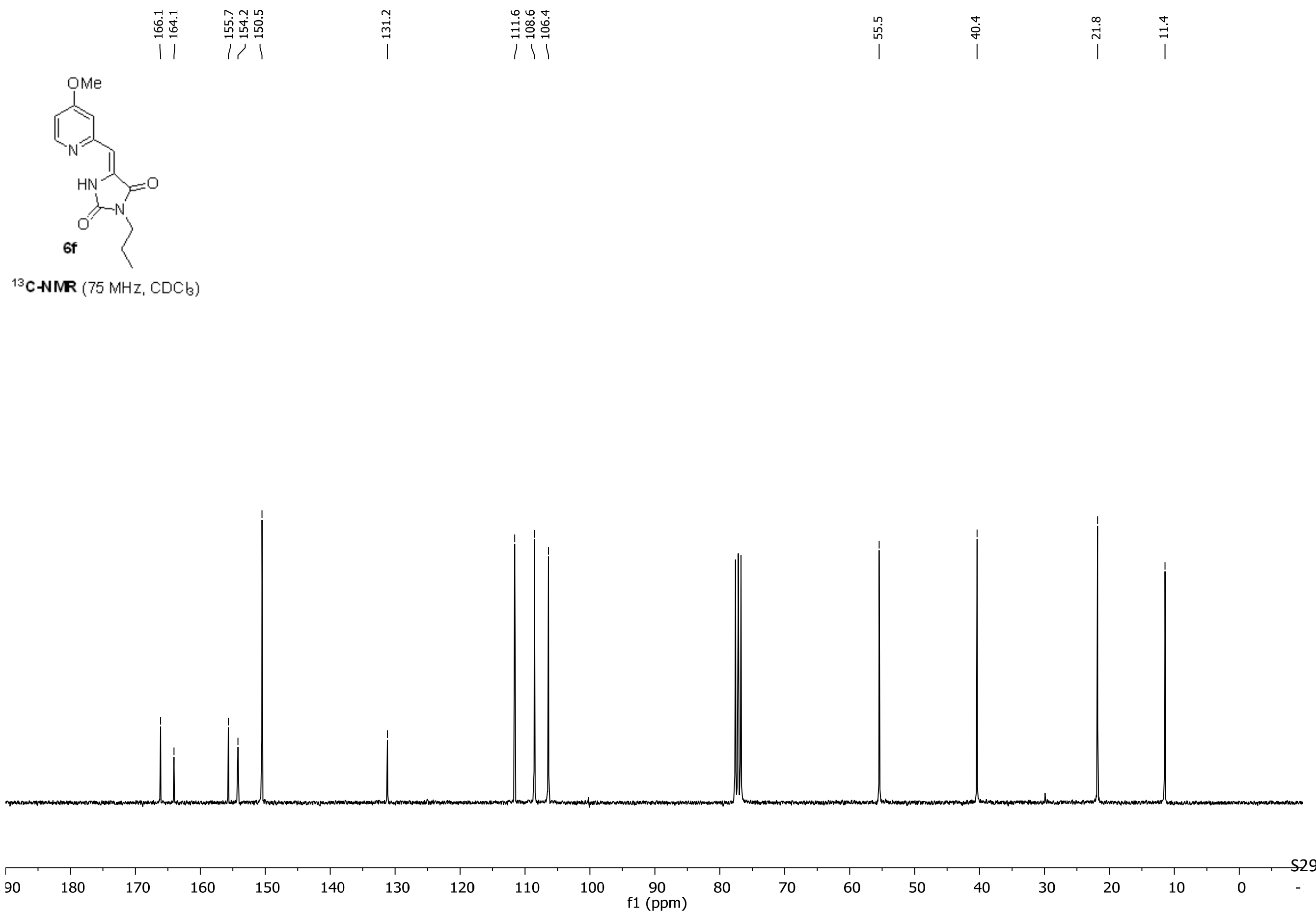
ڤ \& ヘヘヘヘペ。
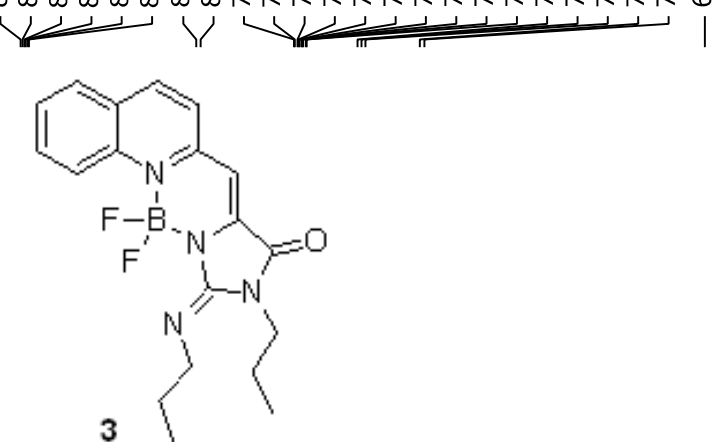

${ }^{1} \mathrm{H}-\mathrm{NMR}\left(500 \mathrm{MHz}, \mathrm{CDCl}_{3}\right)$

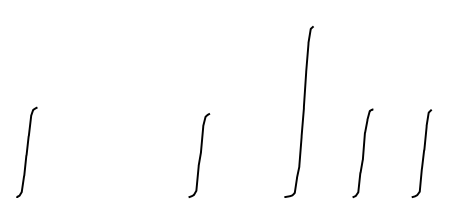

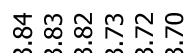

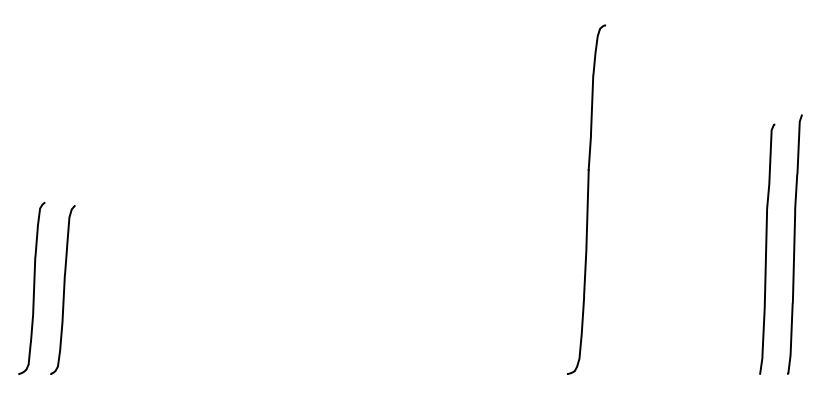

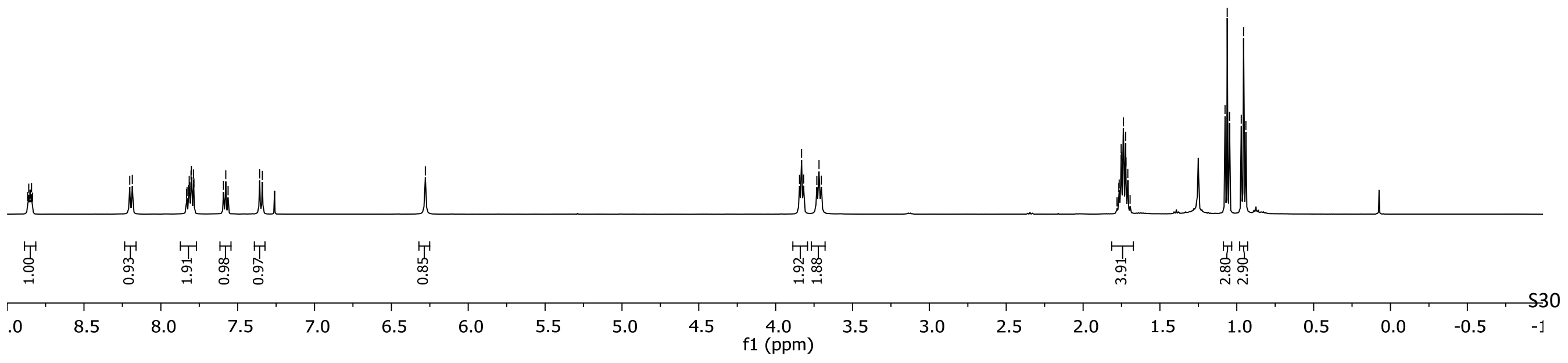




|

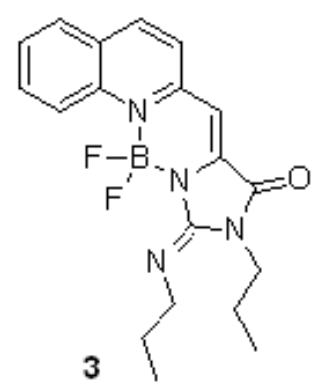

${ }^{13} \mathrm{C}-\mathrm{NMR}\left(126 \mathrm{MHz}_{1} \mathrm{CDCl}_{3}\right)$

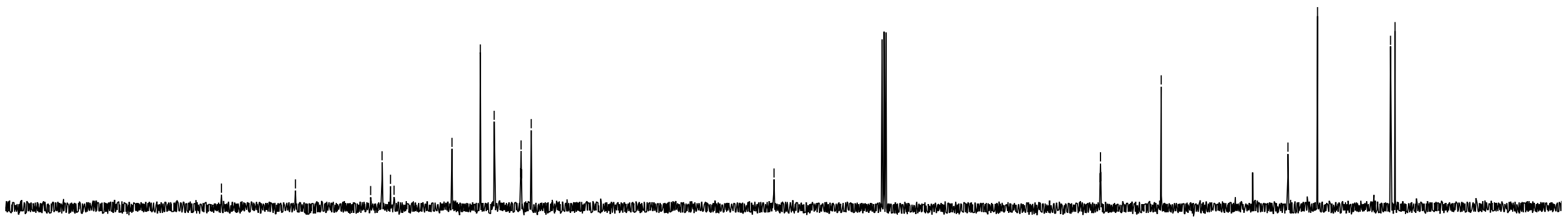

\begin{tabular}{|c|c|c|c|c|c|c|c|c|c|c|c|c|c|c|c|c|c|c|c|}
\hline 90 & 180 & 170 & 160 & 150 & 140 & 130 & 120 & 110 & 100 & $\begin{array}{c}90 \\
\mathrm{f} 1(\mathrm{ppm})\end{array}$ & 80 & 70 & 60 & 50 & 40 & 30 & 20 & 10 & 0 \\
\hline
\end{tabular}




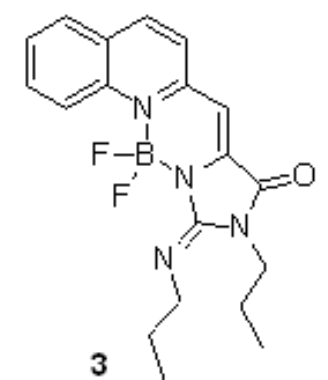

กิ ซึน ํํํ

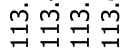

iv'

${ }^{19} \mathrm{~F}-\mathrm{NMR}\left(282 \mathrm{MHz}, \mathrm{CDCl}_{3}\right)$

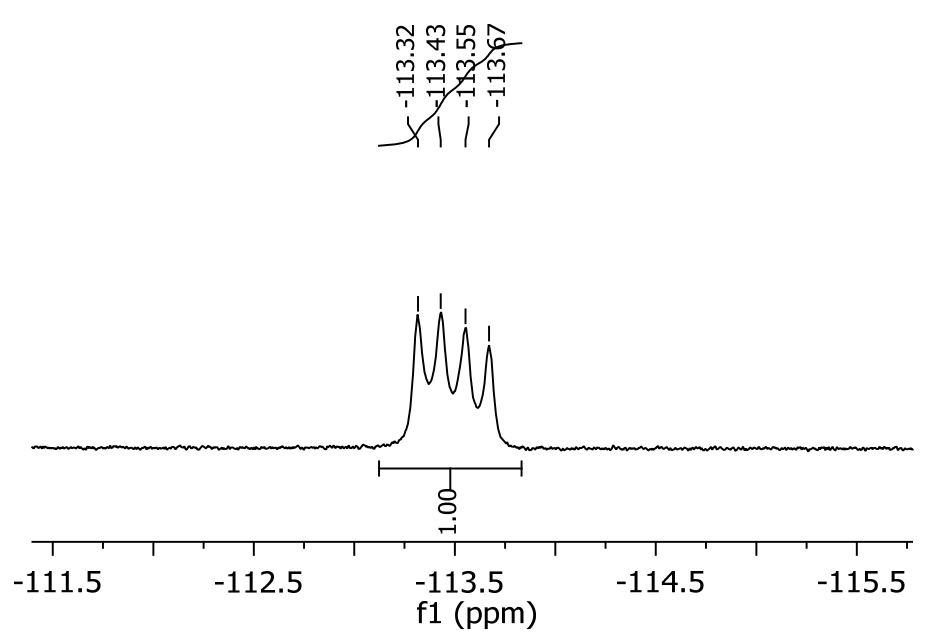

$$
\mid
$$

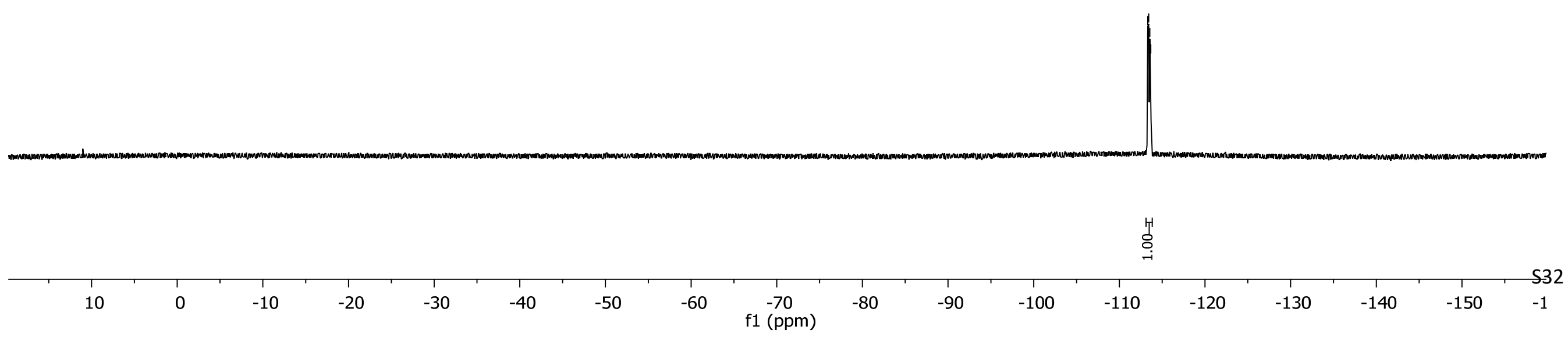



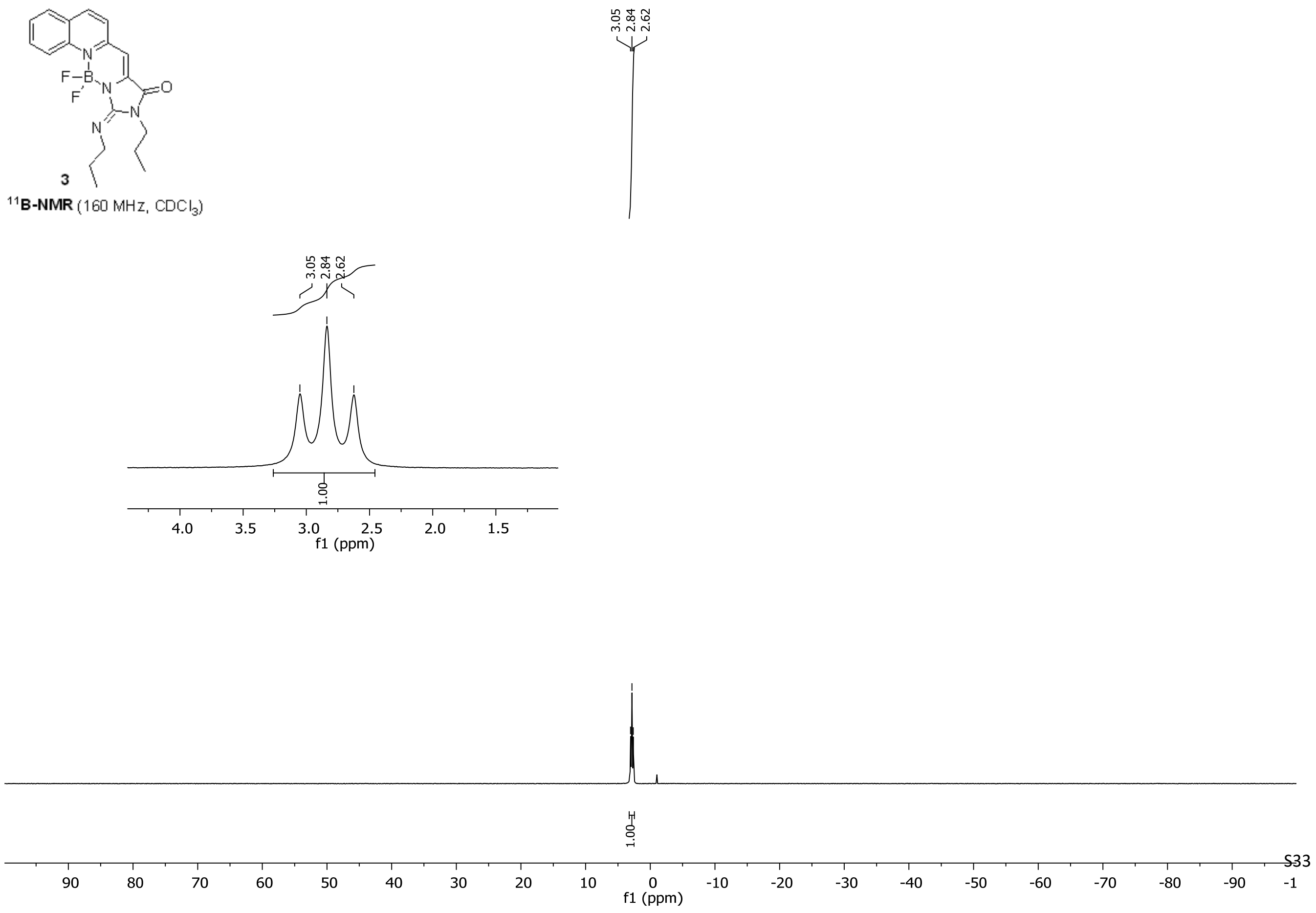


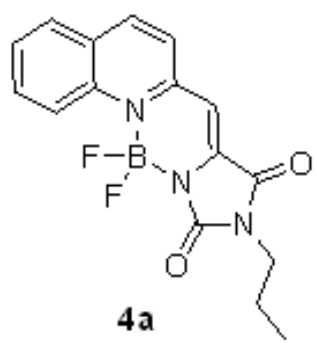

'HANMR (500 MHz, $\left.\mathrm{CDCl}_{3}\right)$
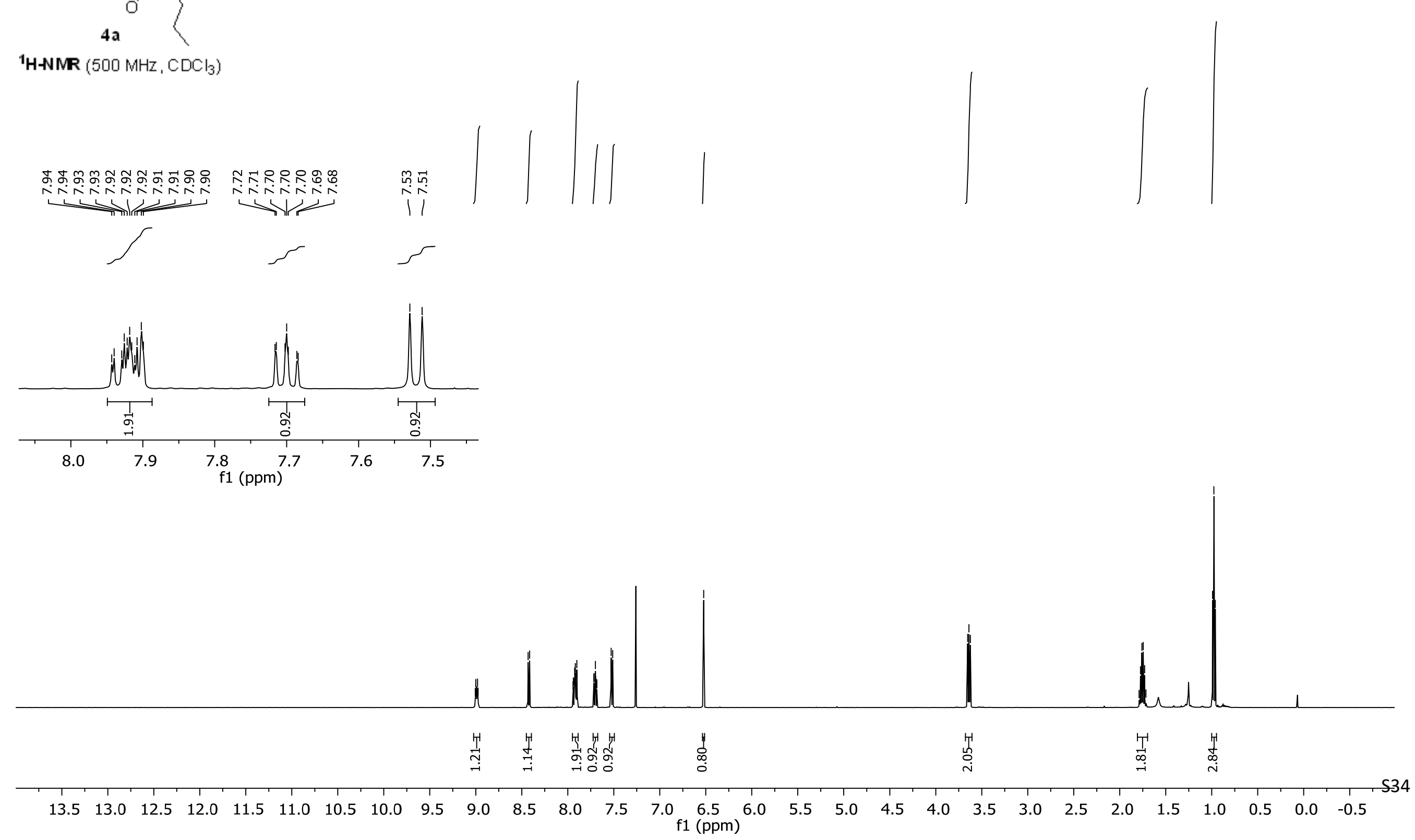




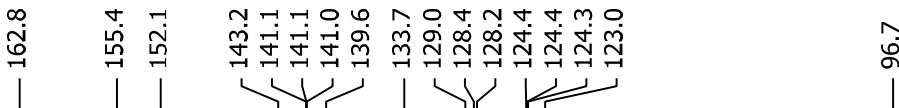

${ }^{13} \mathrm{C}$-NMR $\left(126 \mathrm{MHz}, \mathrm{CDCl}_{3}\right)$
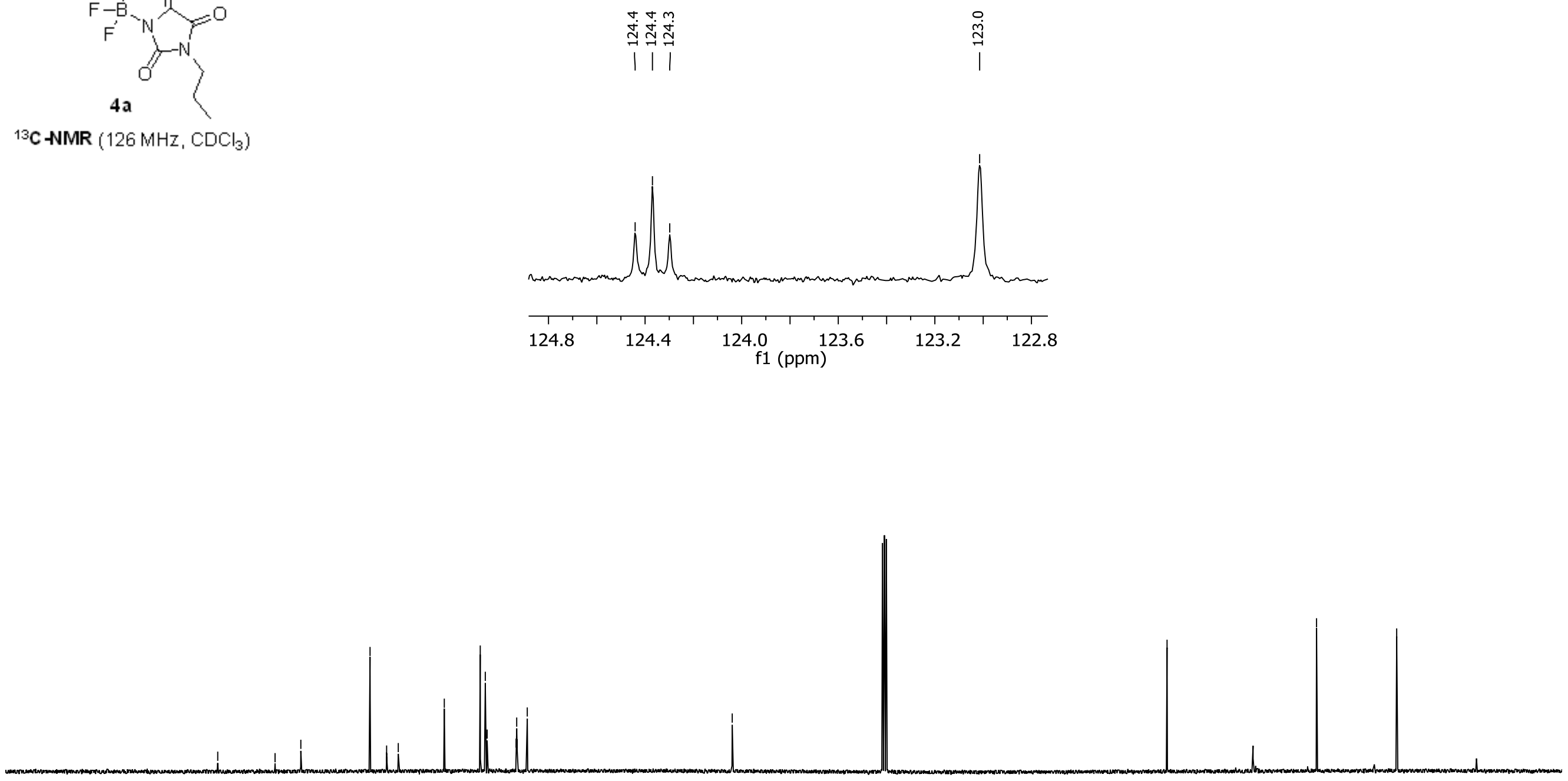

\begin{tabular}{|c|c|c|c|c|c|c|c|c|c|c|c|c|c|c|c|c|c|c|c|}
\hline \multirow[b]{2}{*}{90} & 1 & 1 & 1 & 1 & $T$ & $\sqrt{1}$ & $T$ & $T$ & T & $T$ & $T$ & 1 & $T$ & $T$ & T & $T$ & $T$ & $T$ & $T$ \\
\hline & 180 & 170 & 160 & 150 & 140 & 130 & 120 & 110 & 100 & $\begin{array}{c}90 \\
\mathrm{f} 1(\mathrm{ppm})\end{array}$ & 80 & 70 & 60 & 50 & 40 & 30 & 20 & 10 & 0 \\
\hline
\end{tabular}




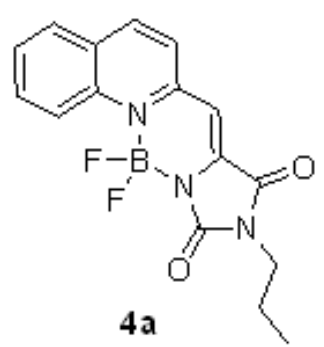

${ }^{19}$ F-NMR $\left(282 \mathrm{MHz}_{1} \mathrm{CDCl}_{3}\right)$
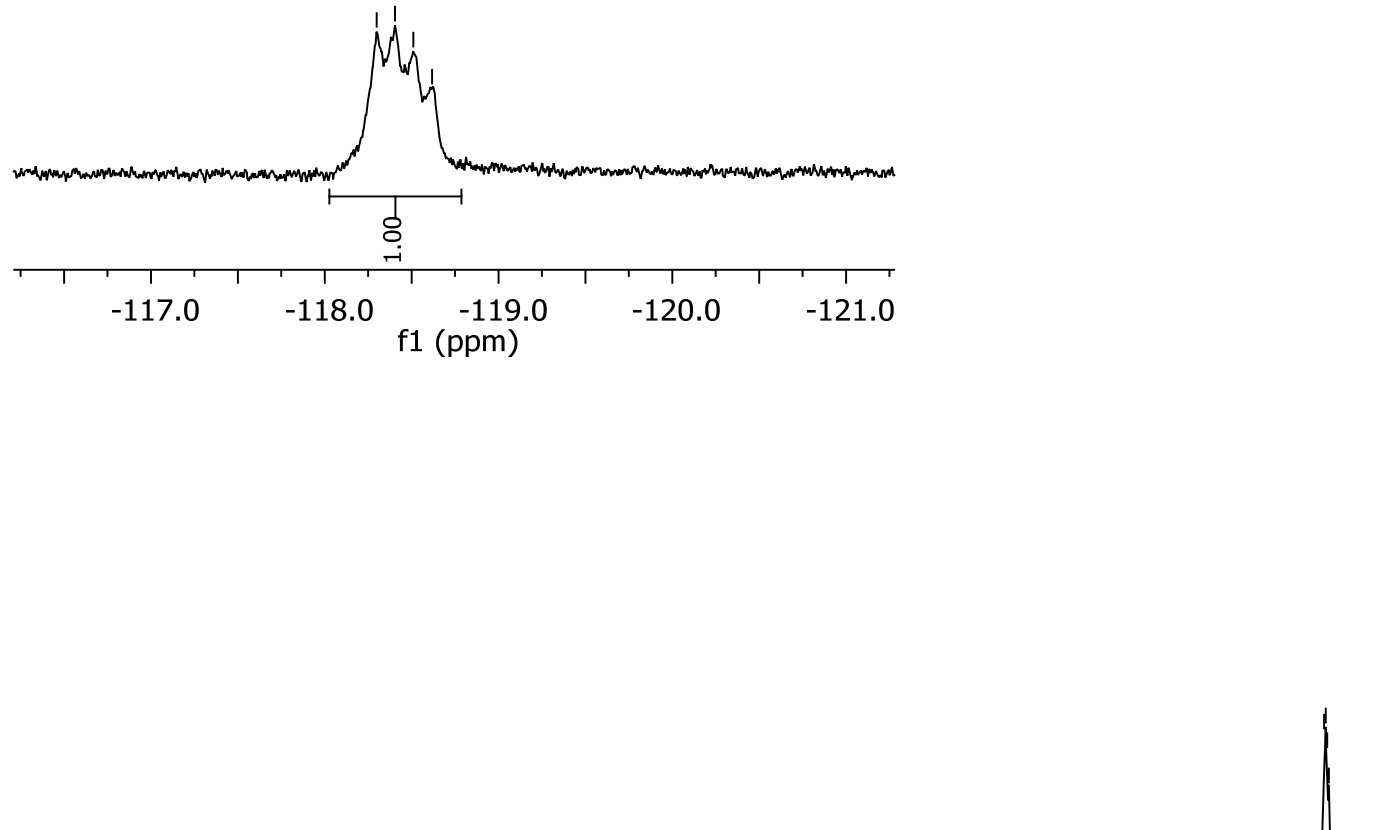

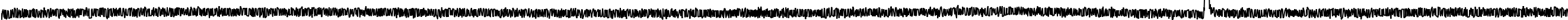

\begin{tabular}{|c|c|c|c|c|c|c|c|c|c|c|c|c|c|c|c|c|c|}
\hline & & & & & & & & & & & & & ه্] & & & & \\
\hline 10 & 0 & -10 & -20 & $\begin{array}{c}1 \\
-30\end{array}$ & -40 & $\begin{array}{c}1 \\
-50\end{array}$ & -60 & $\begin{array}{c}-70 \\
\mathrm{f} 1(\mathrm{ppm})\end{array}$ & -80 & -90 & -100 & -110 & -120 & -130 & -140 & -150 & -1 \\
\hline
\end{tabular}




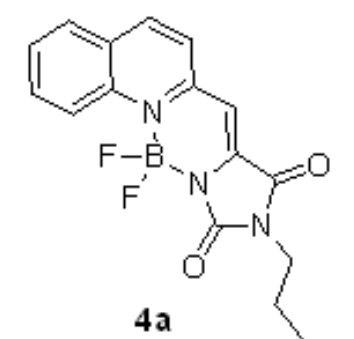

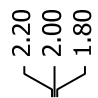

11B -NMR (160 MHz, CDCl$)$
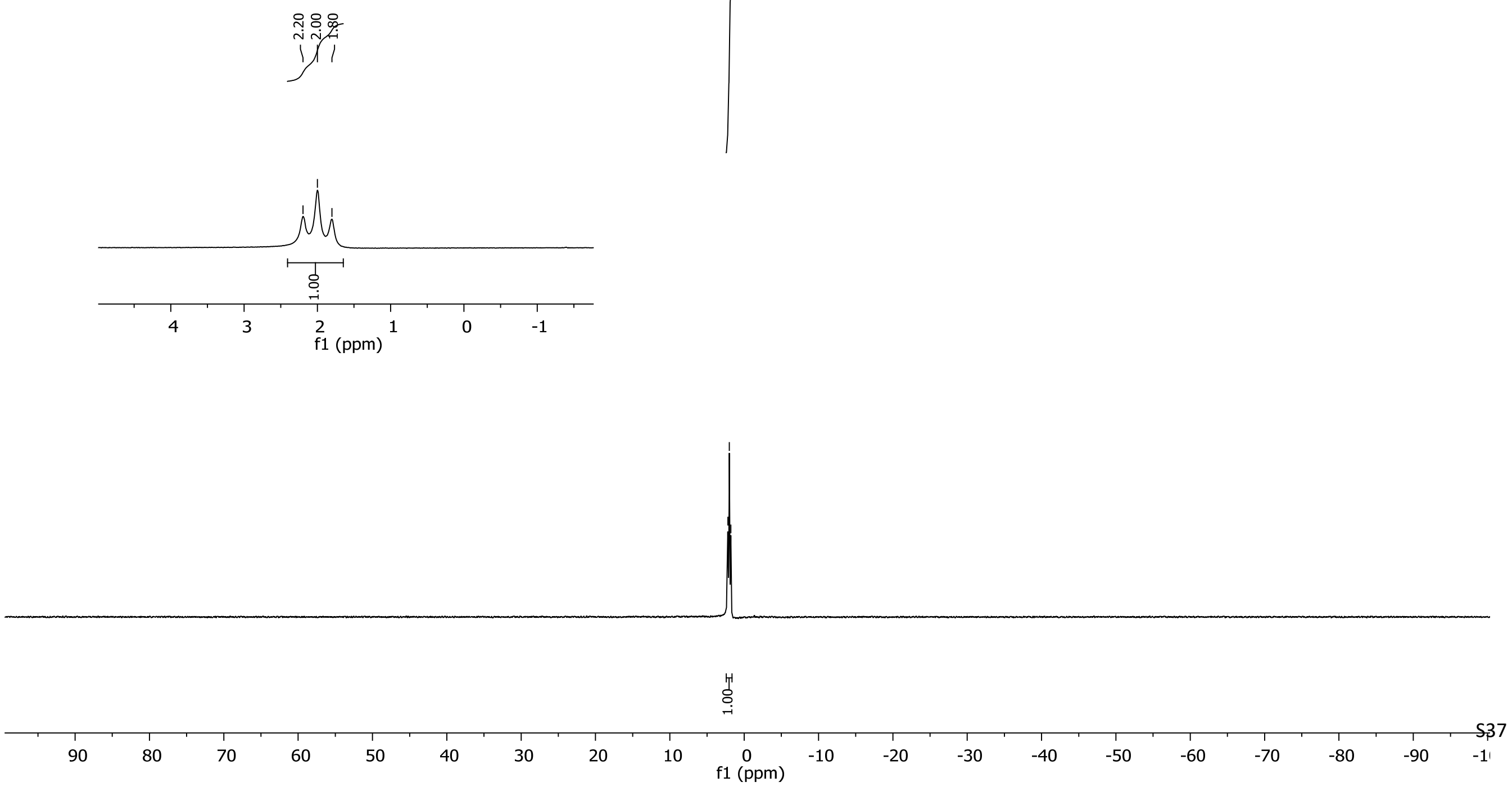


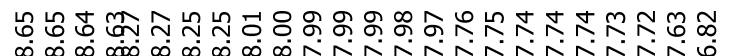

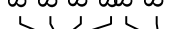

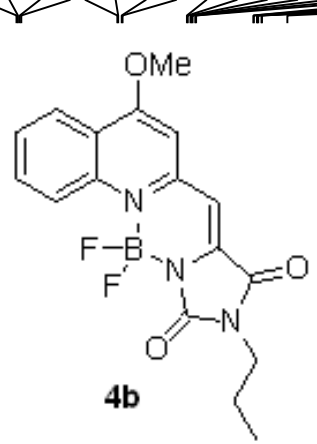

${ }^{1}$ H-NMR $\left(500 \mathrm{MHz}, \mathrm{DMSO}-\alpha_{6}\right)$

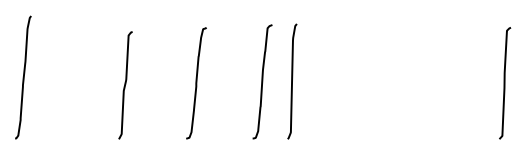

I

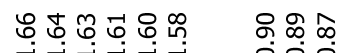

调
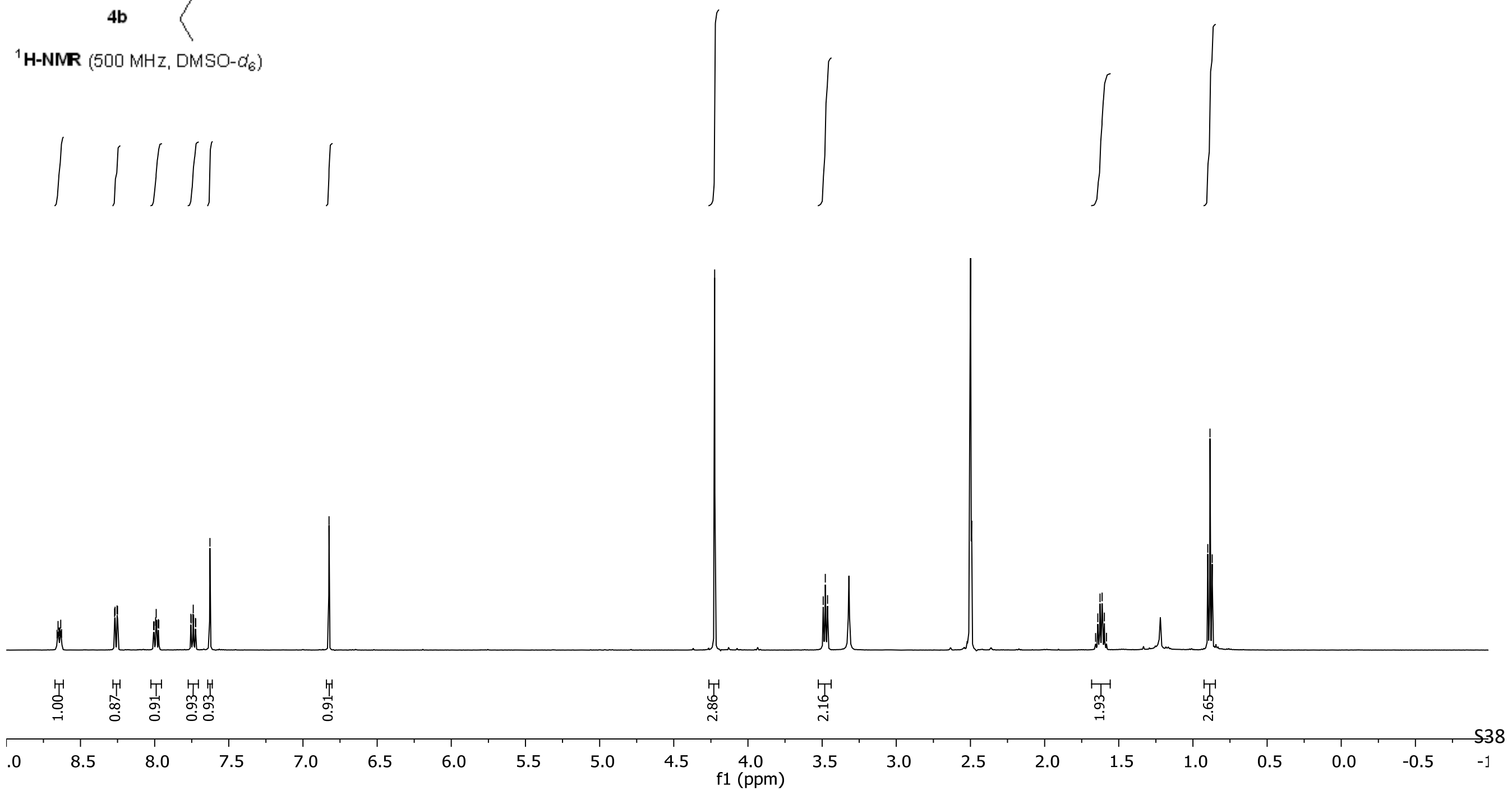


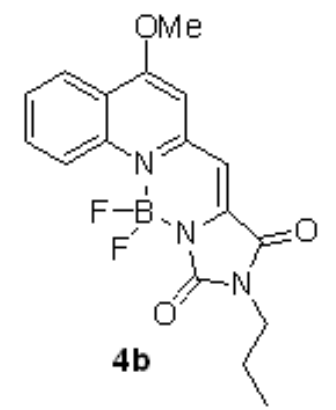

\section{สำสำสำ}

${ }^{13} \mathrm{C}-\mathrm{NMR}\left(126 \mathrm{MHz}, \mathrm{DMSO}-\alpha_{6}\right)$

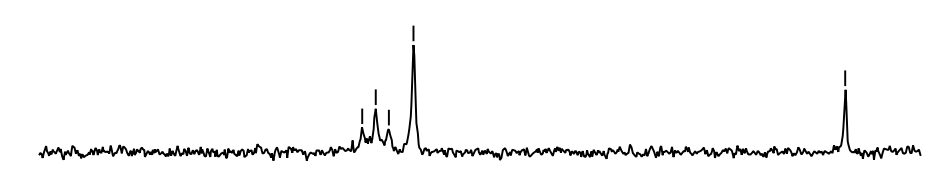

$\begin{array}{lllllllllll}124.5 & 124.0 & 123.5 & 123.0 & 122.5 & 122.0 & 121.5 & 121.0 & 120.5 & 120.0\end{array}$

$$
\text { f1 (ppm) }
$$

\section{\ulcorner}




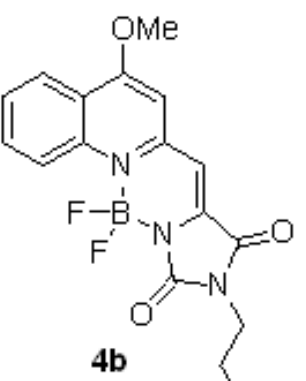

${ }^{19}$ F-NMR $\left(282 \mathrm{MHz}, \mathrm{DMSO}-d_{6}\right)$

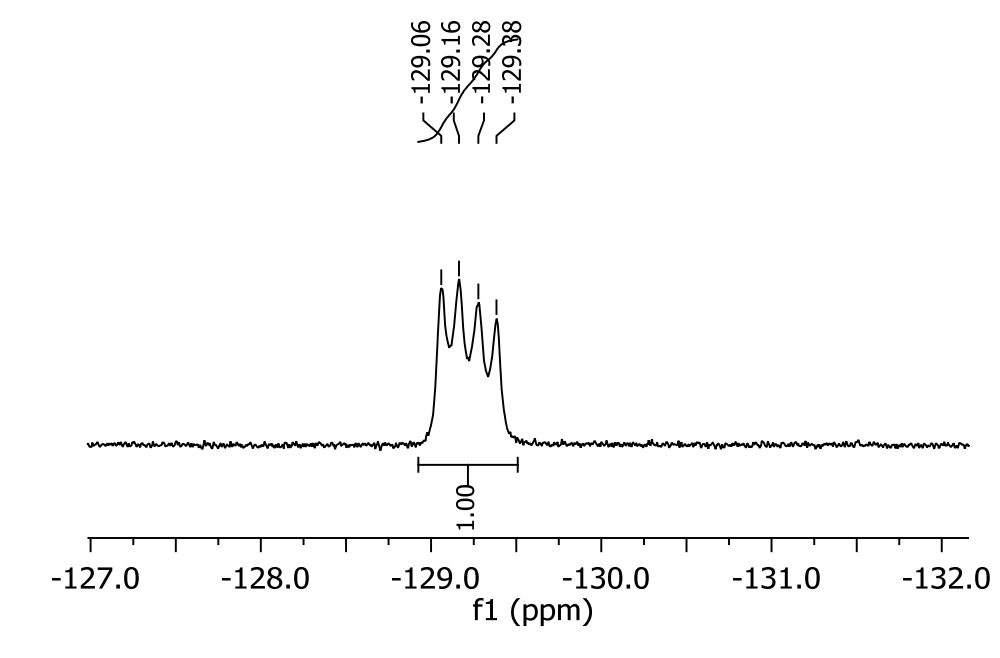

(2)






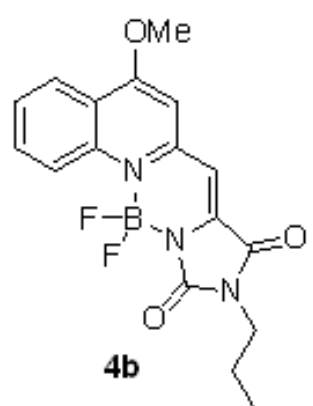

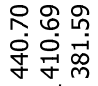

${ }^{11}$ B-NMR $\left(160 \mathrm{MHz}, \mathrm{DMSO}-\alpha_{6}\right)$
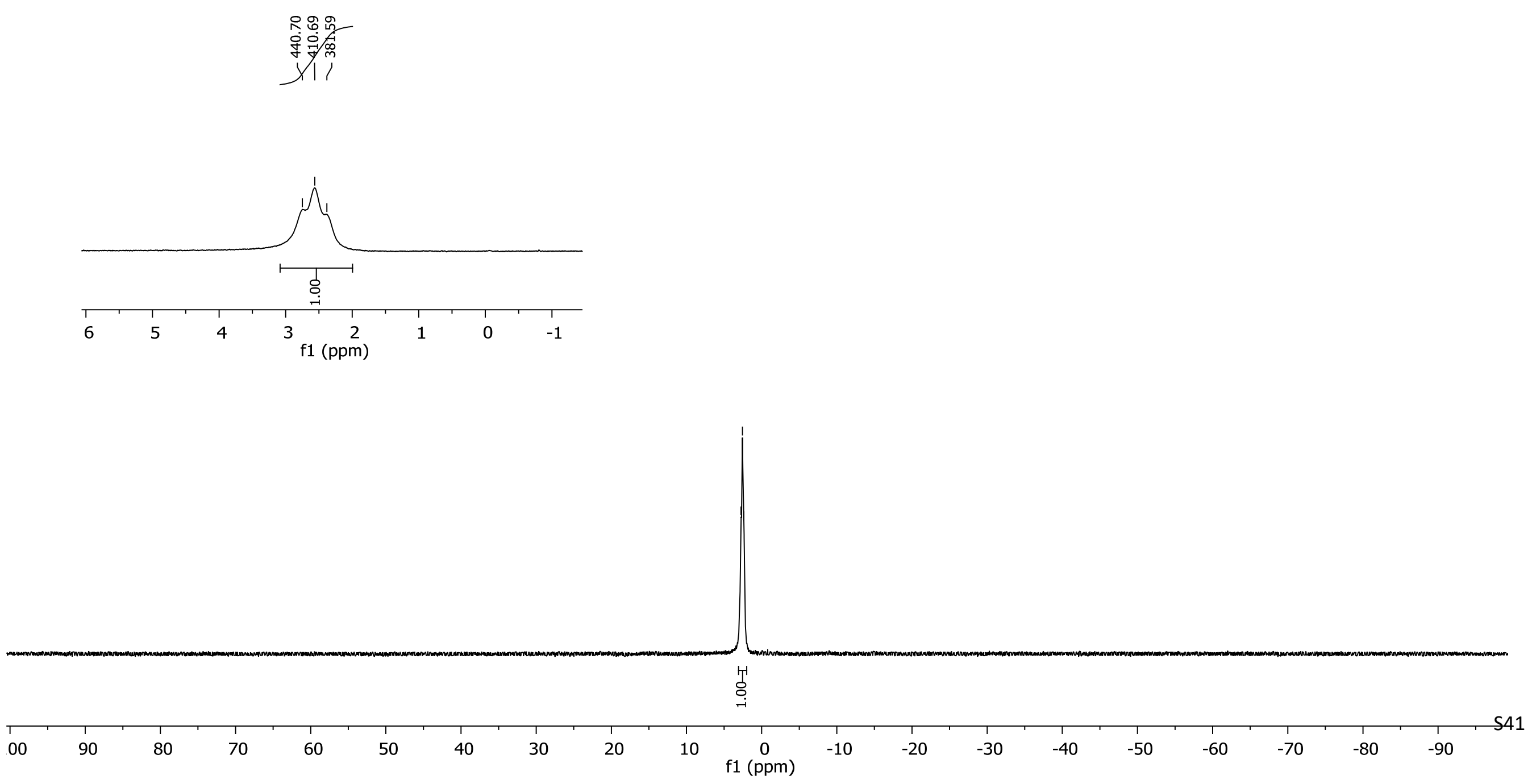


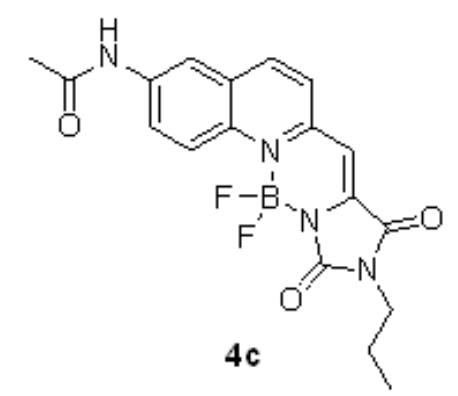

${ }^{1}$ H-NMR $\left(500 \mathrm{MHz}, \mathrm{DMSO}-d_{6}\right)$

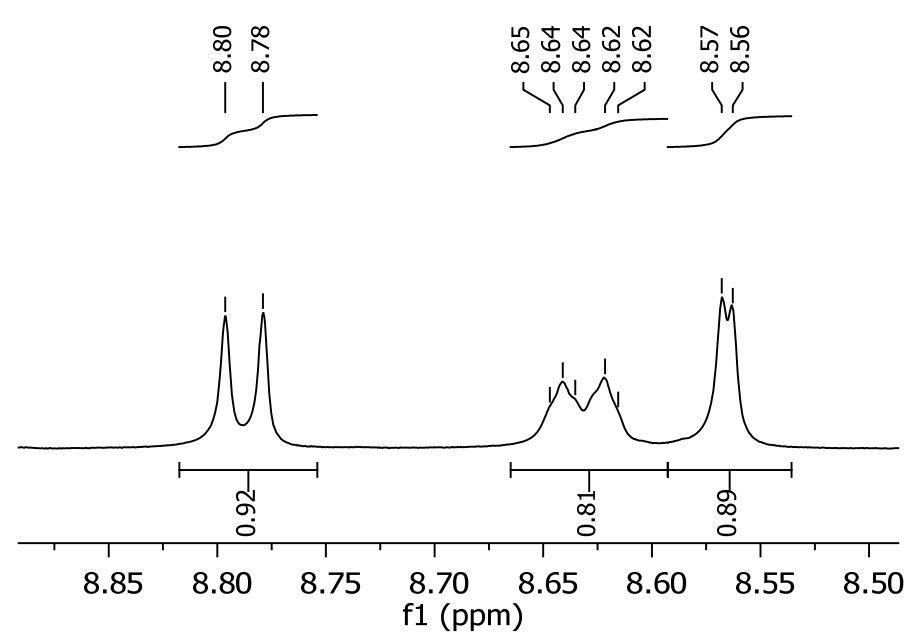

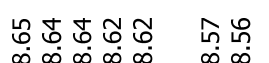
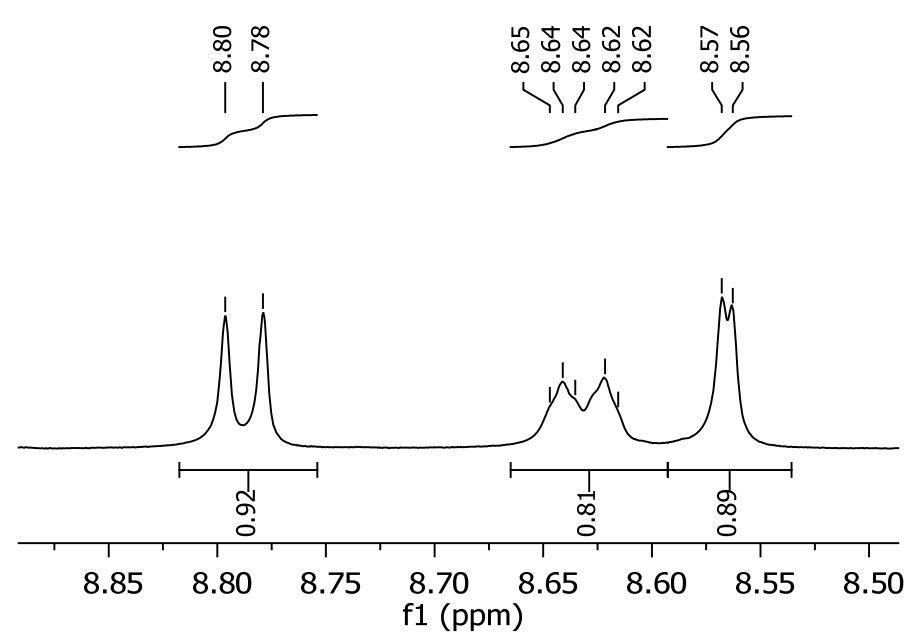
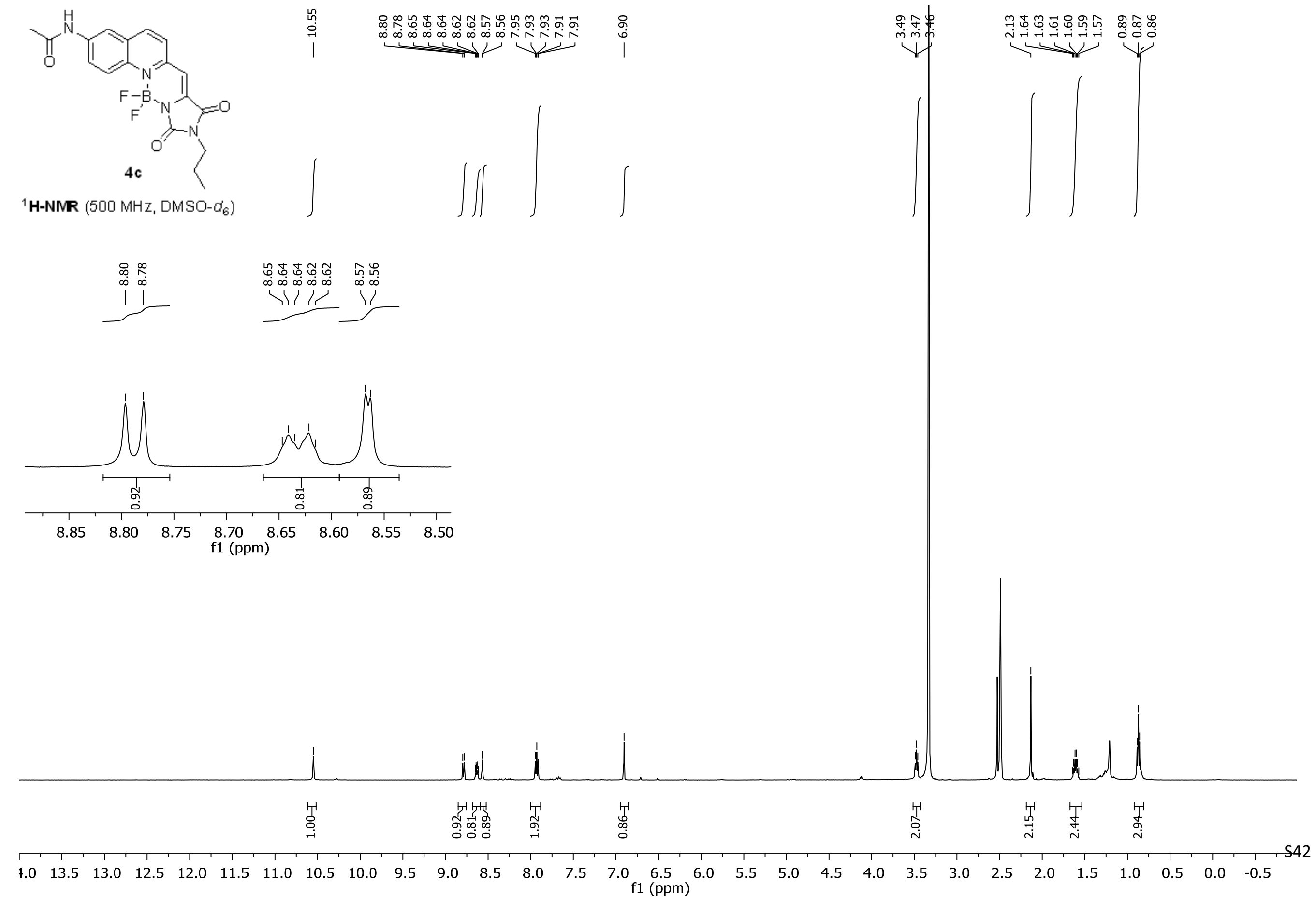


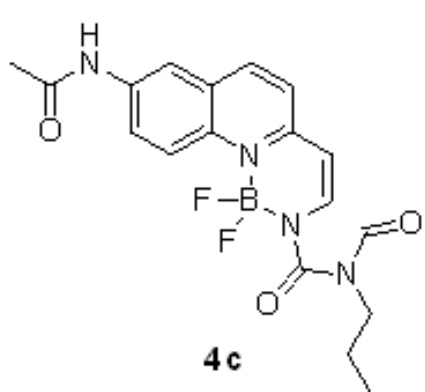



${ }^{13} \mathrm{C}-\mathrm{NMR}\left(75 \mathrm{MHz}, \mathrm{DMSO}-d^{6}\right)$ 


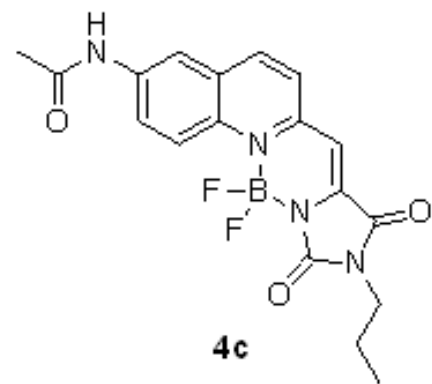

${ }^{19}$ F-NMR $\left(282 \mathrm{MHz}, \mathrm{DMSO}-\alpha_{6}\right)$
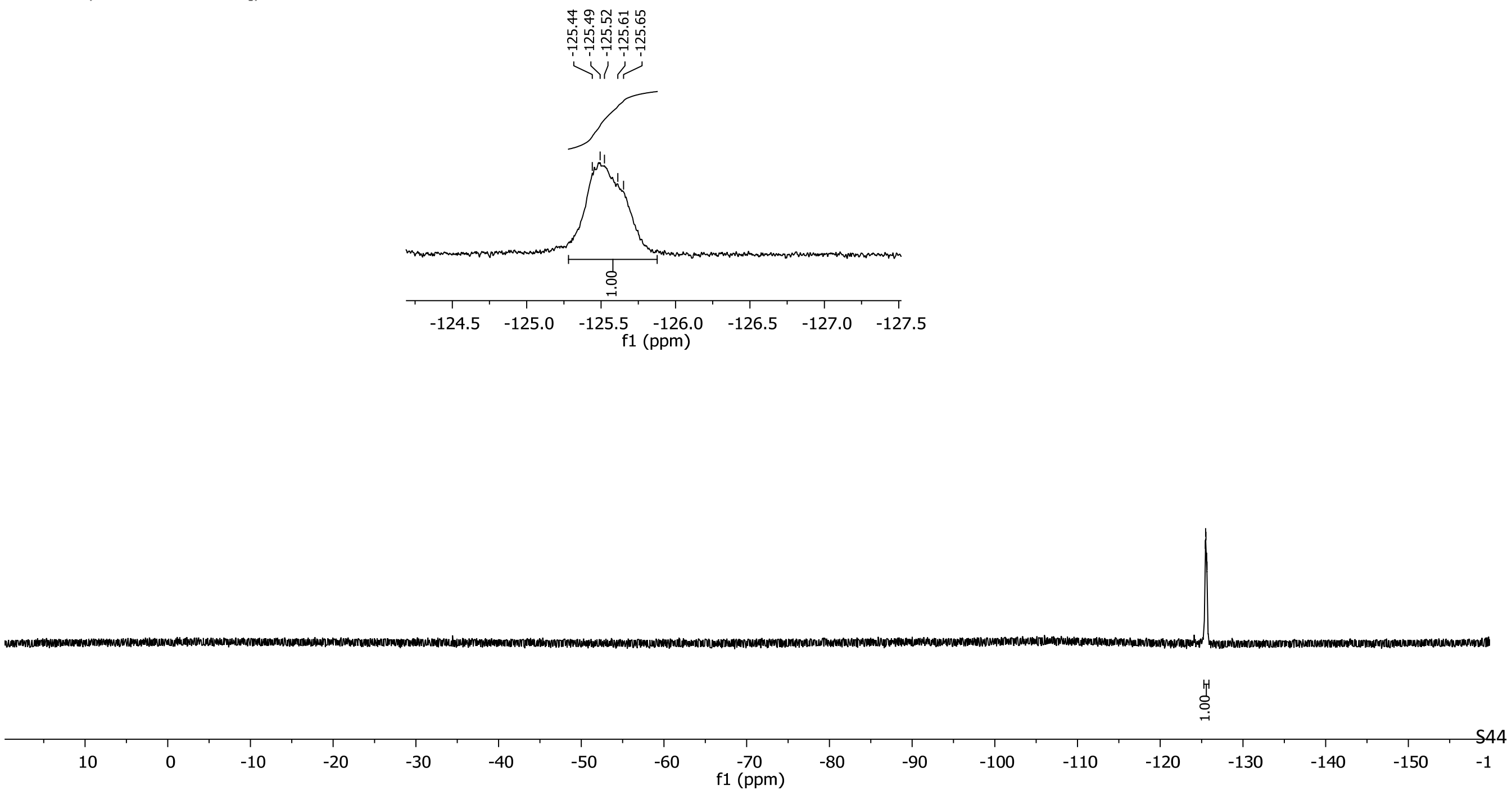


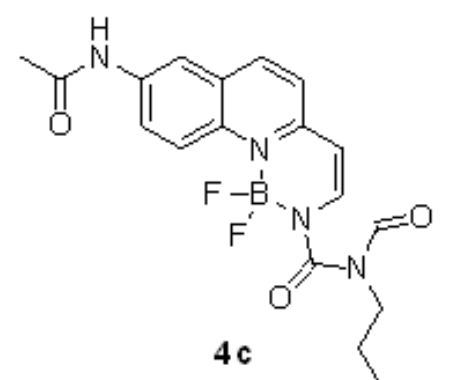

${ }^{11}$ B- NMR (160 MHz, DMSO- $\left.d^{6}\right)$

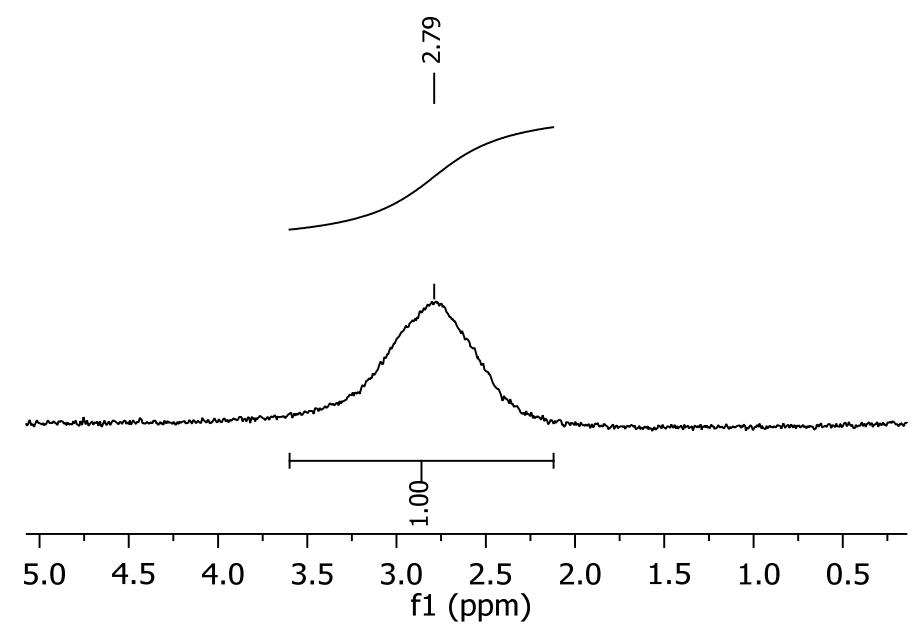




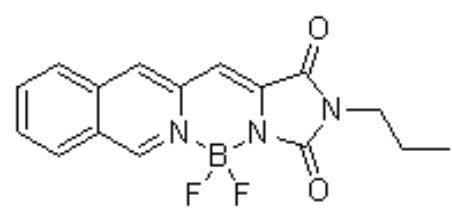

$4 d$

${ }^{1}$ H-NMR (300 MHz, DMSO- $\left.d_{6}\right)$
\&

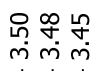

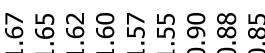

西
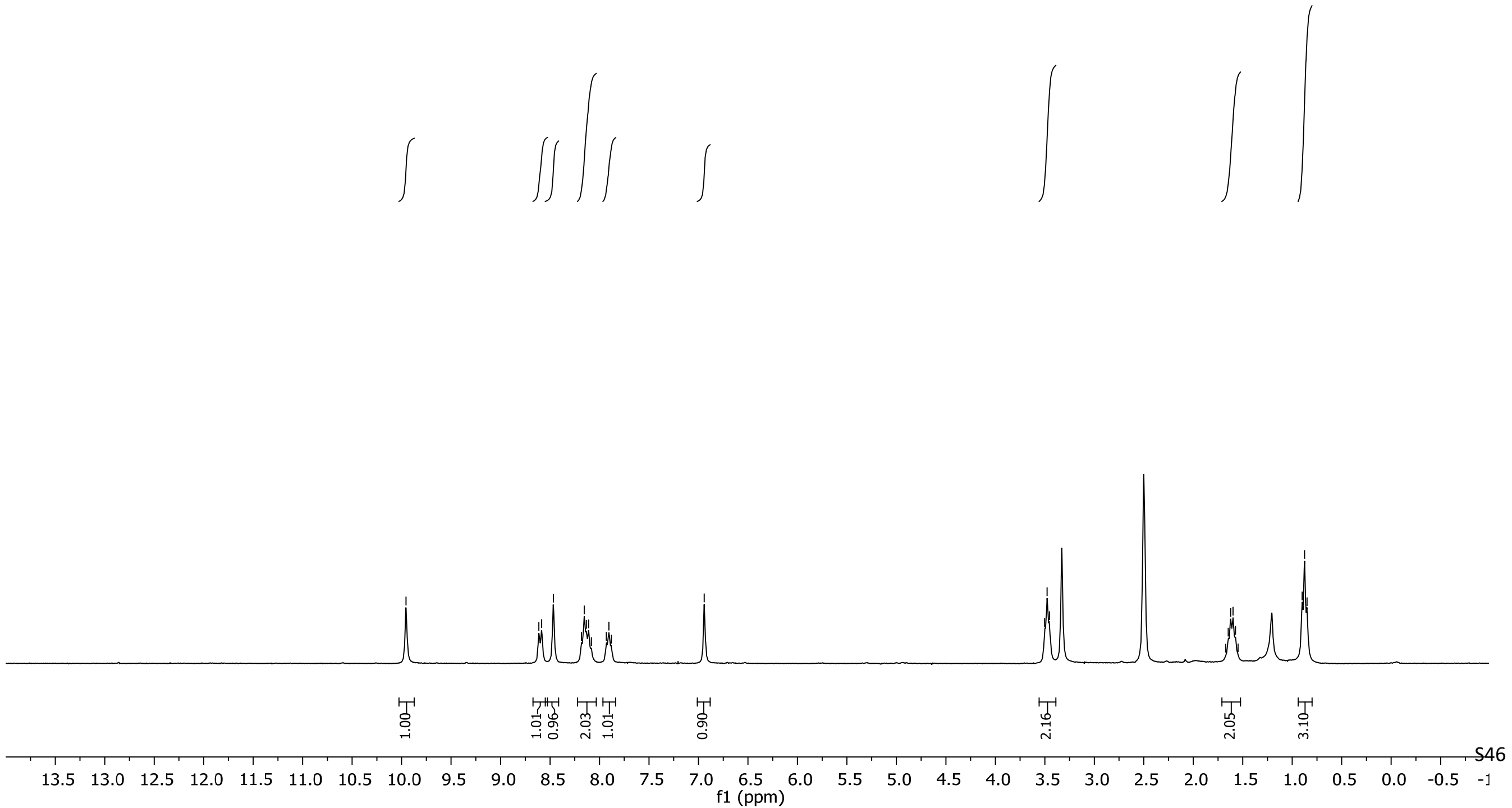


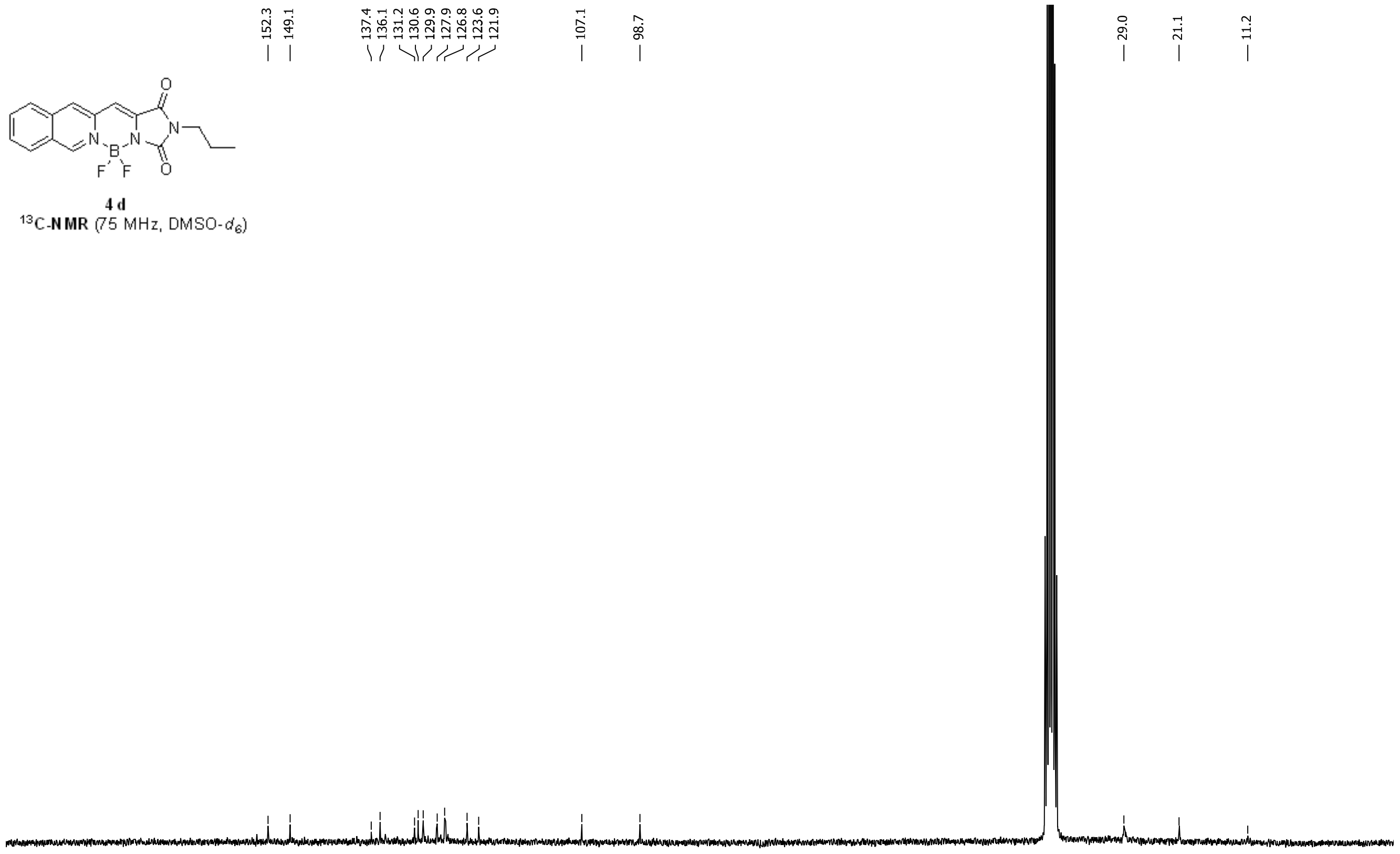

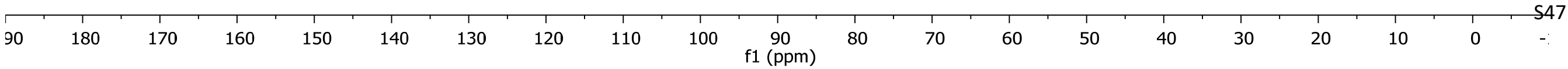




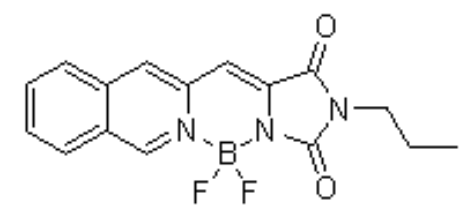

${ }^{19}$ F-NMR (282 MHz, DMSO- $\left.d_{6}\right)$
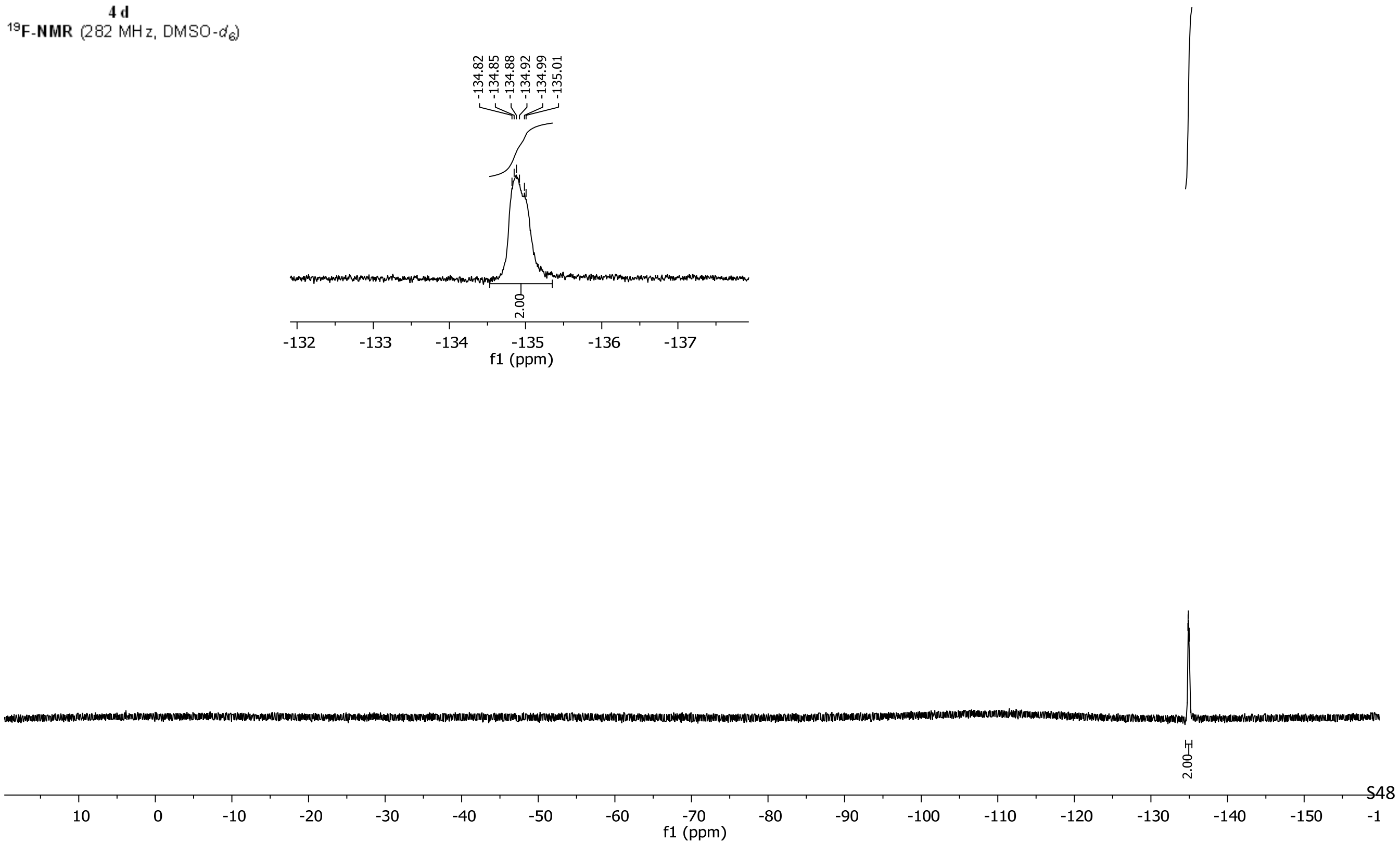


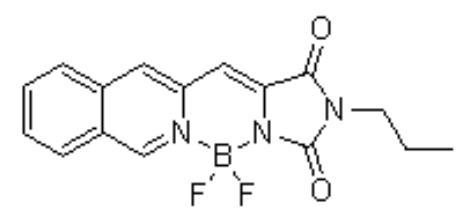

${ }^{11}$ B -N MR (160 MHz, DMSO- $\left.d_{6}\right)$
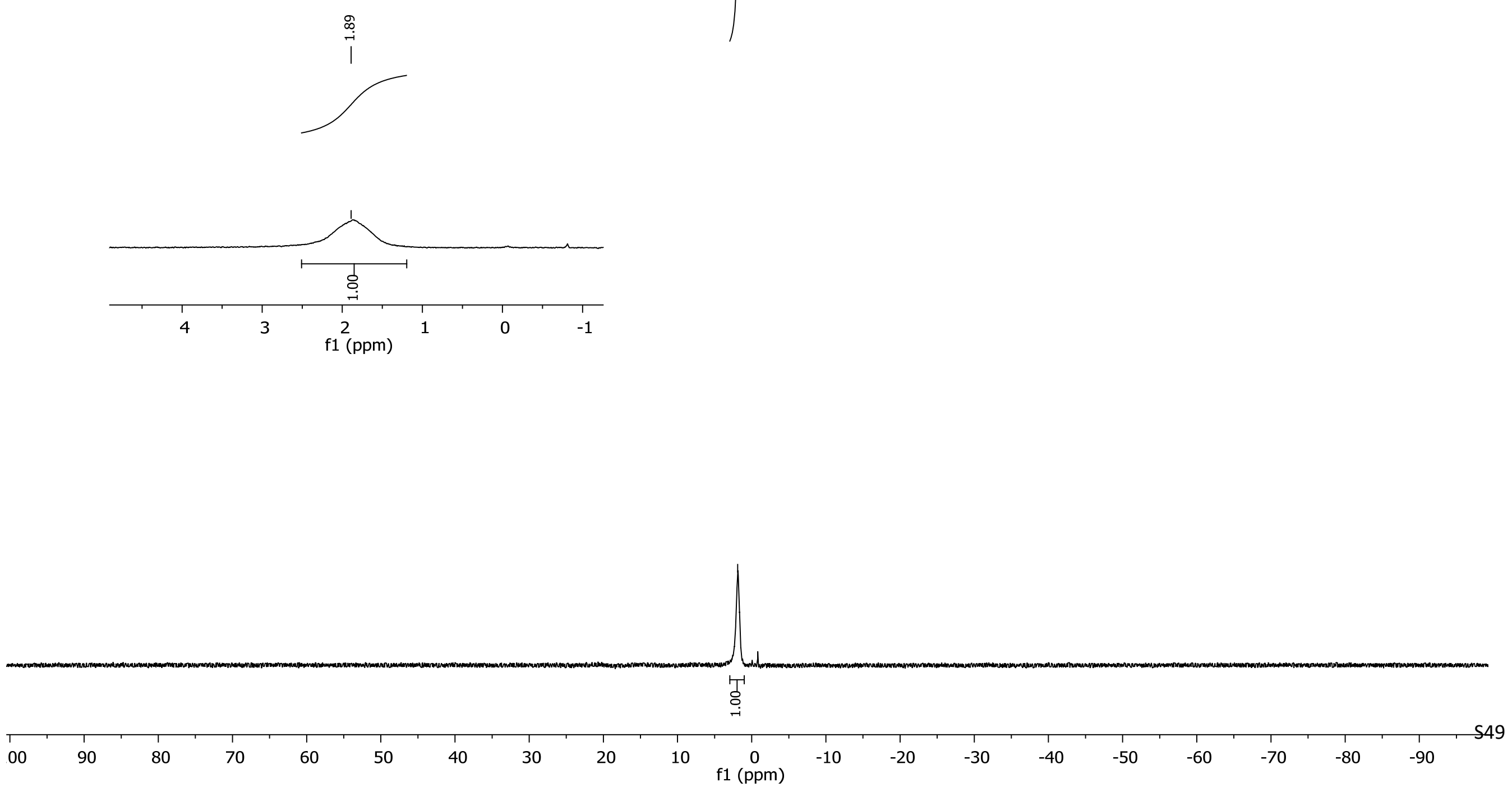




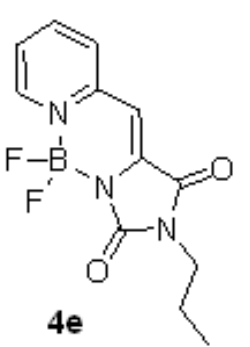

${ }^{1}$ H-NMR (300 MHz, $\left.\mathrm{CDCl}_{3}\right)$
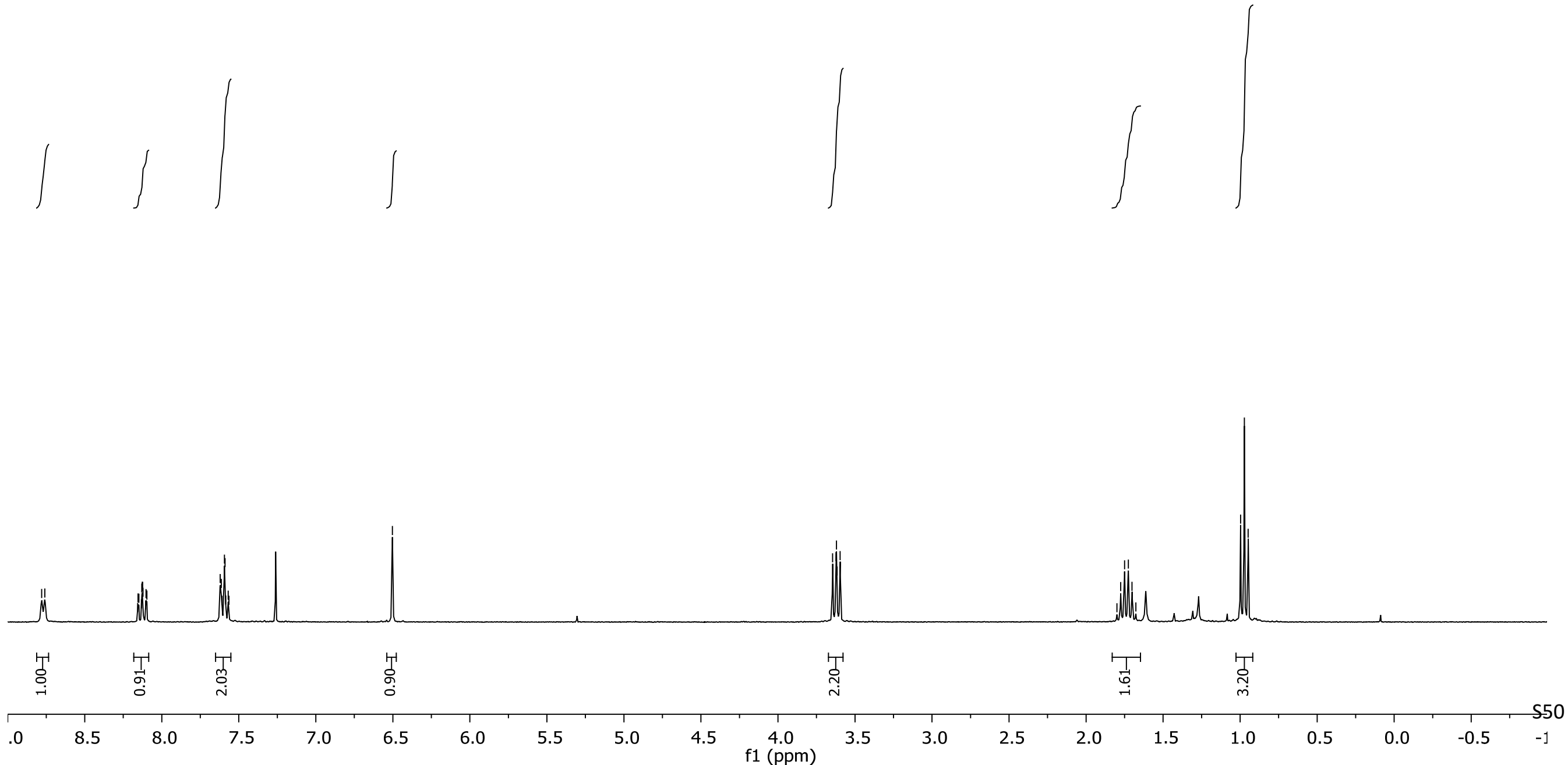


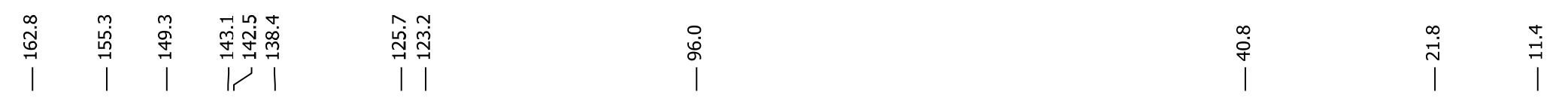



${ }^{13} \mathrm{C}-\mathrm{NMR}\left(75 \mathrm{MHz}, \mathrm{CDCl}_{3}\right)$
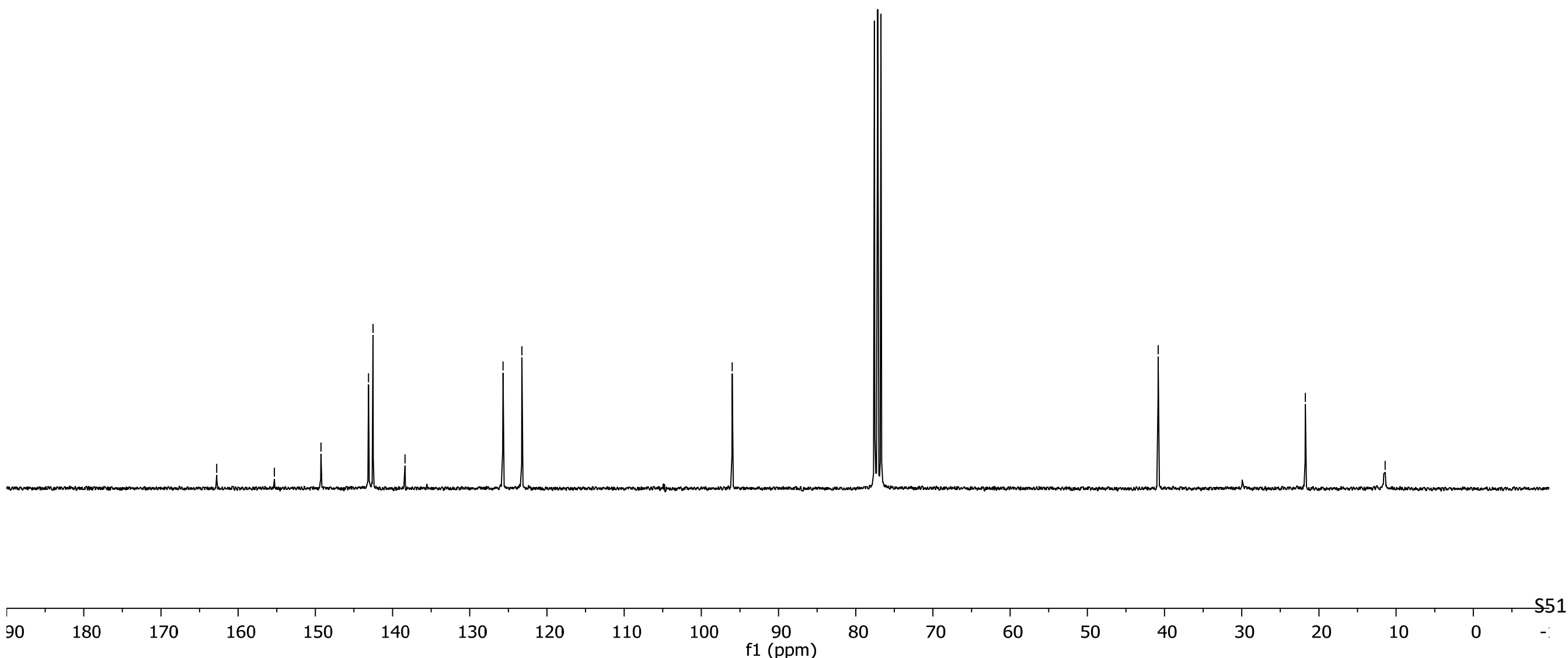


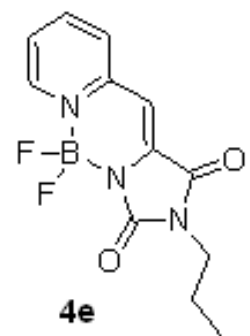

${ }^{19}$ F-NMR $\left(282 \mathrm{MHz}_{1} \mathrm{CDCl}_{3}\right)$
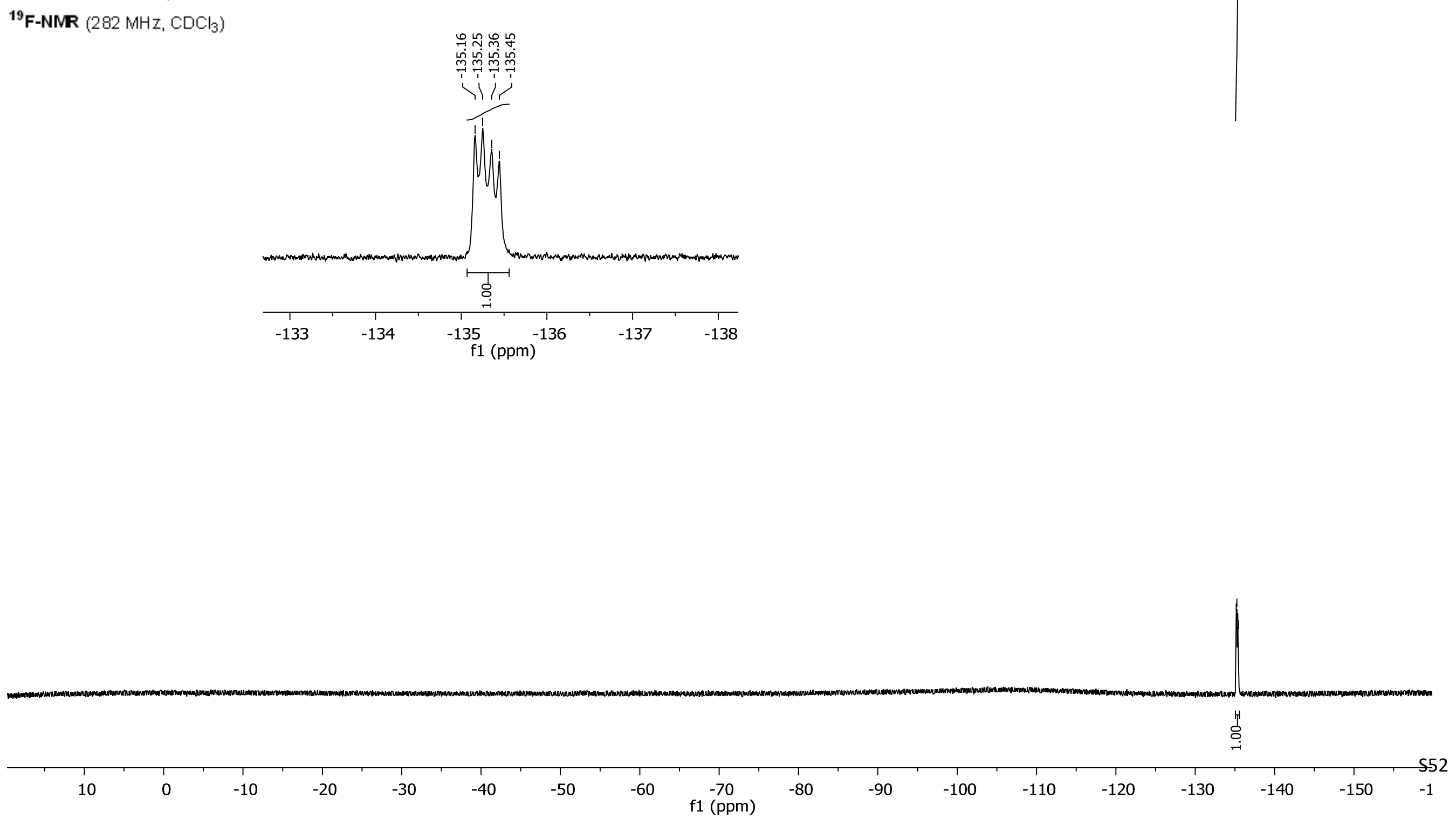


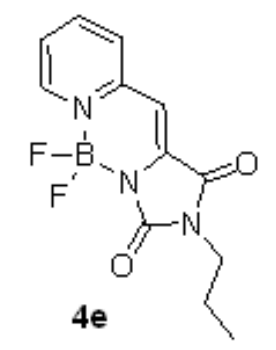

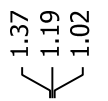

${ }^{11}$ B-NMR $\left(160 \mathrm{MHz}, \mathrm{CDCl}_{3}\right)$
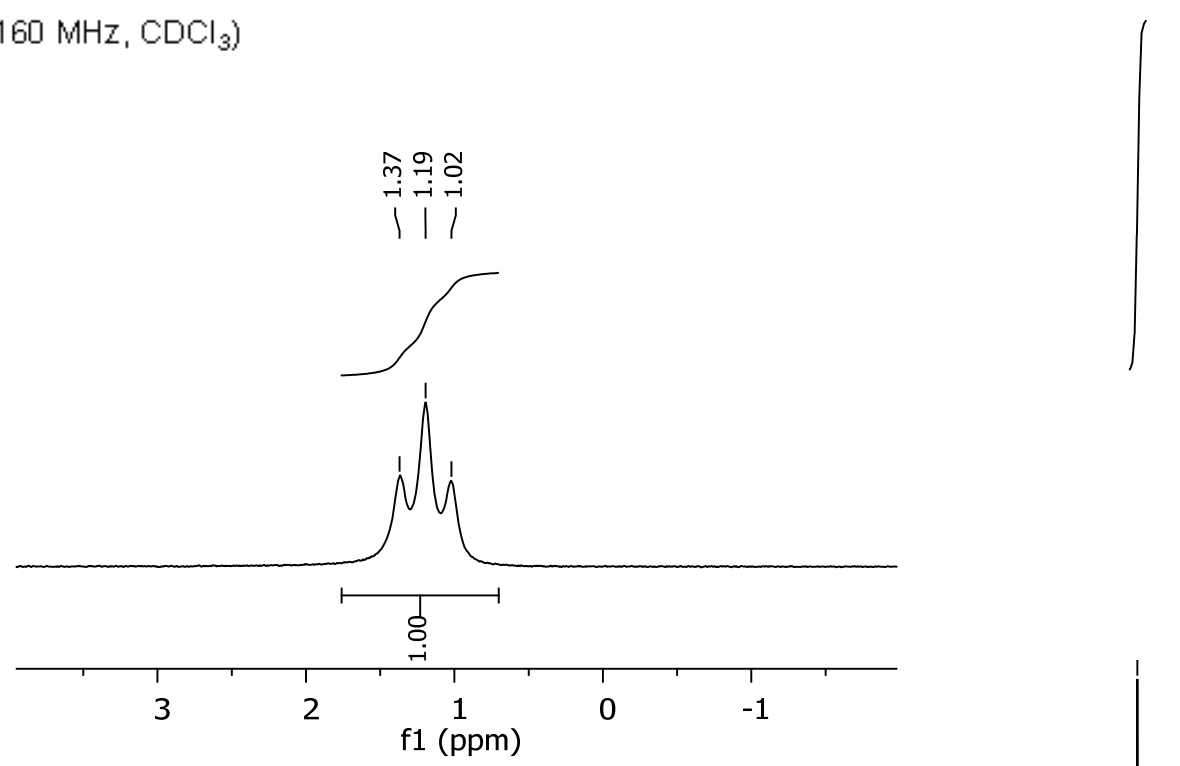

$\stackrel{+}{3}$

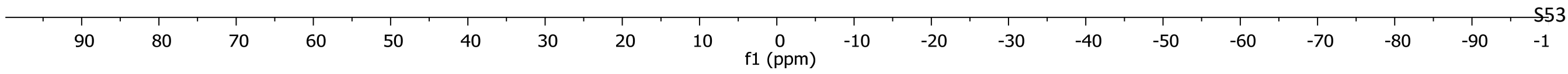




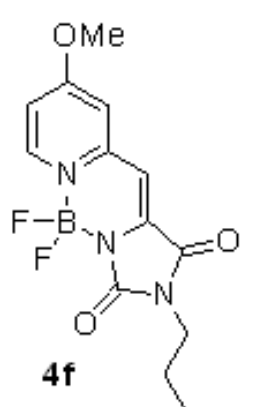

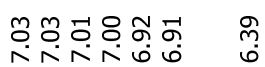

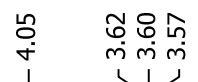

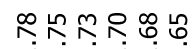

คิํํㅇํำ

।

+

1/1

iा

${ }^{1} \mathrm{H}-\mathrm{NMR}\left(300 \mathrm{MHz}, \mathrm{CDCl}_{3}\right)$
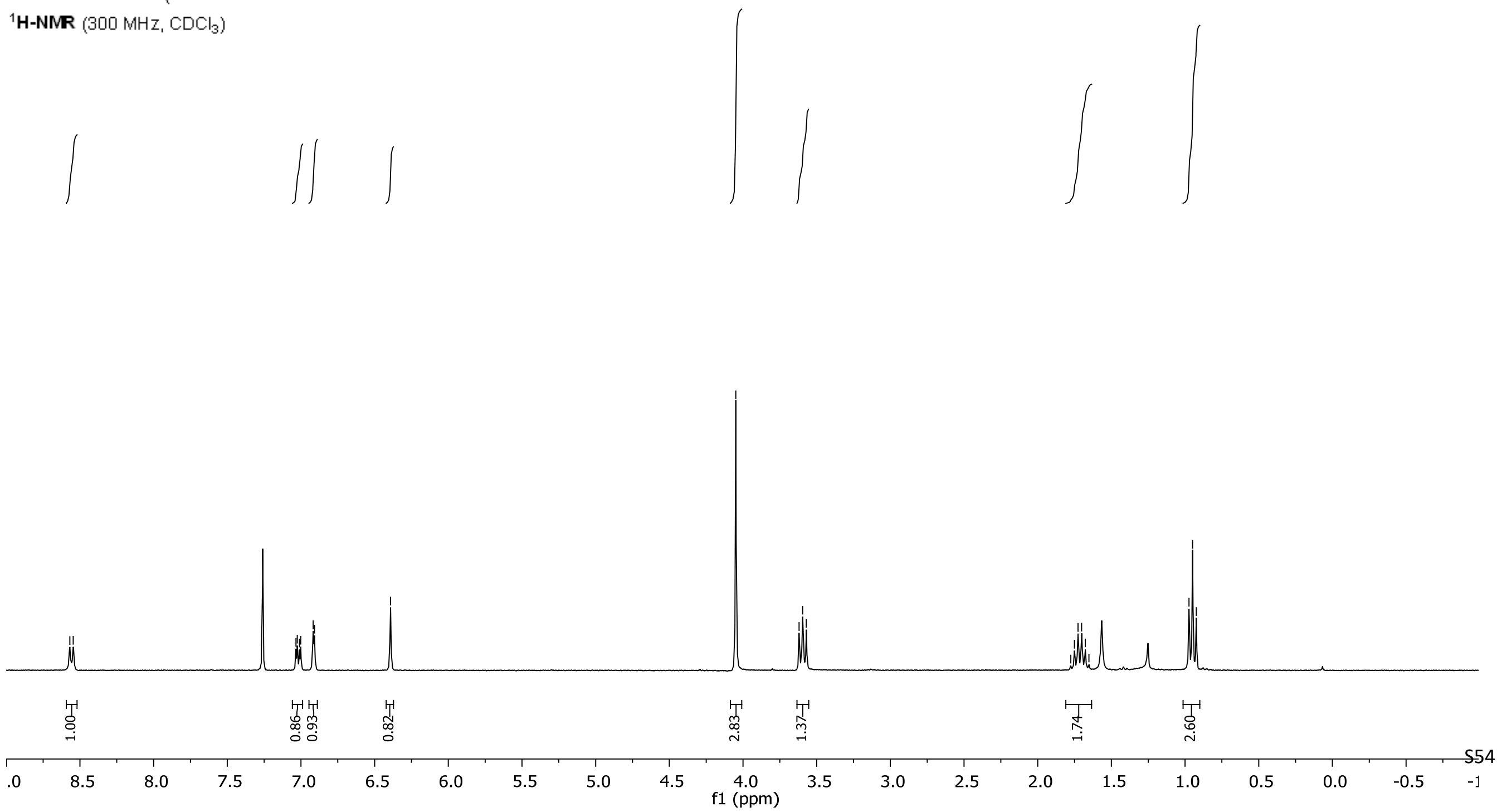


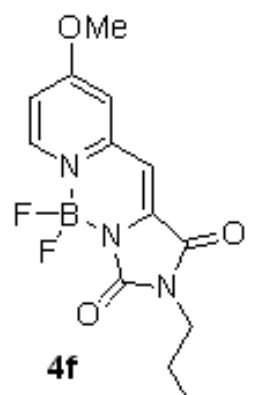

${ }^{13} \mathrm{C}-\mathrm{NMR}\left(126 \mathrm{MHz}, \mathrm{CDCl}_{3}\right)$ 


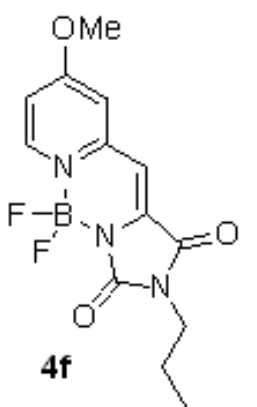

${ }^{19}$ F-NMR $\left(282 \mathrm{MHz}_{1} \mathrm{CDCl}_{3}\right)$
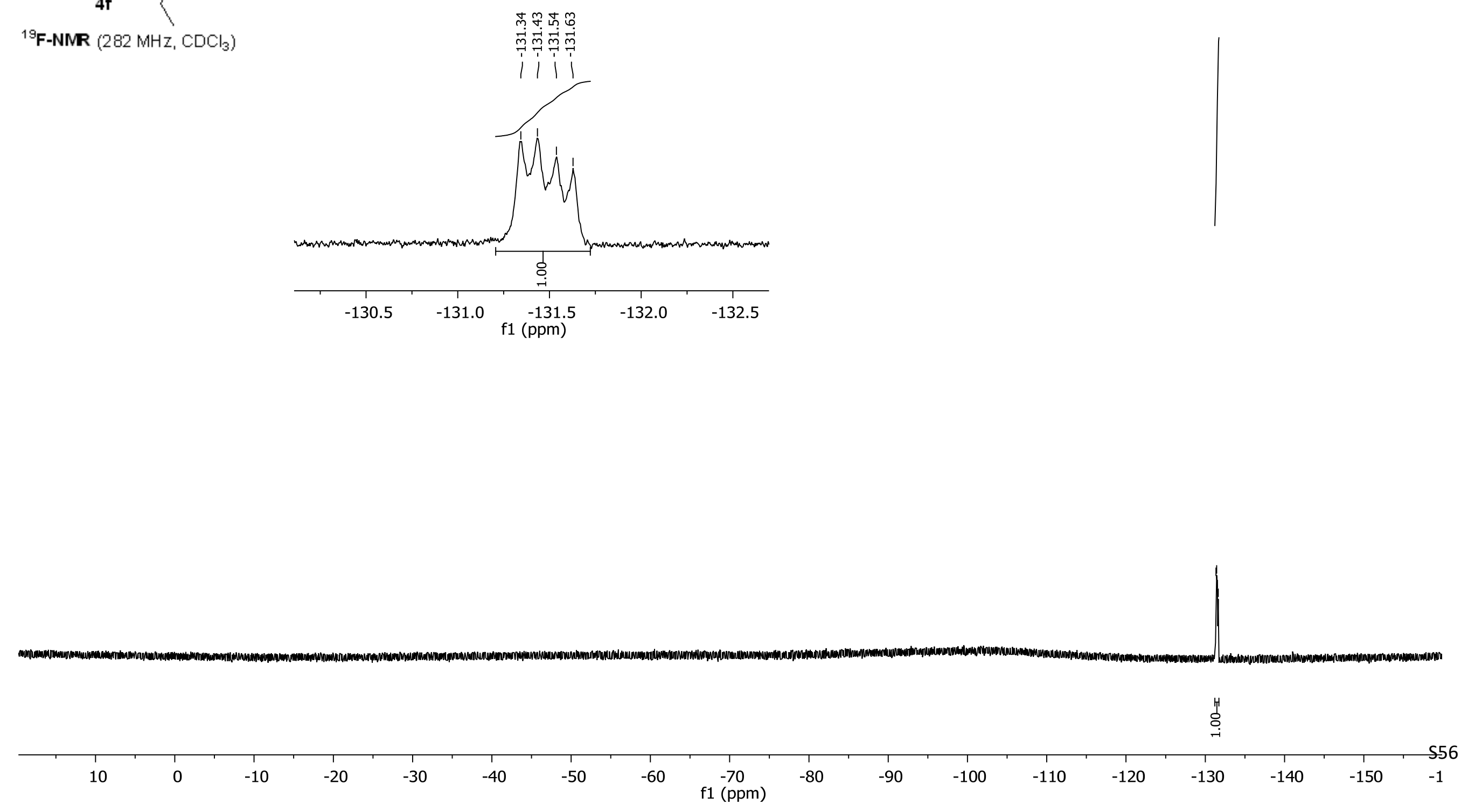


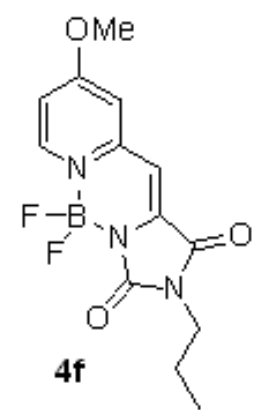

용

${ }^{11}$ B-NMR ( $\left.160 \mathrm{MHz}_{1} \mathrm{CDCl}_{3}\right)$
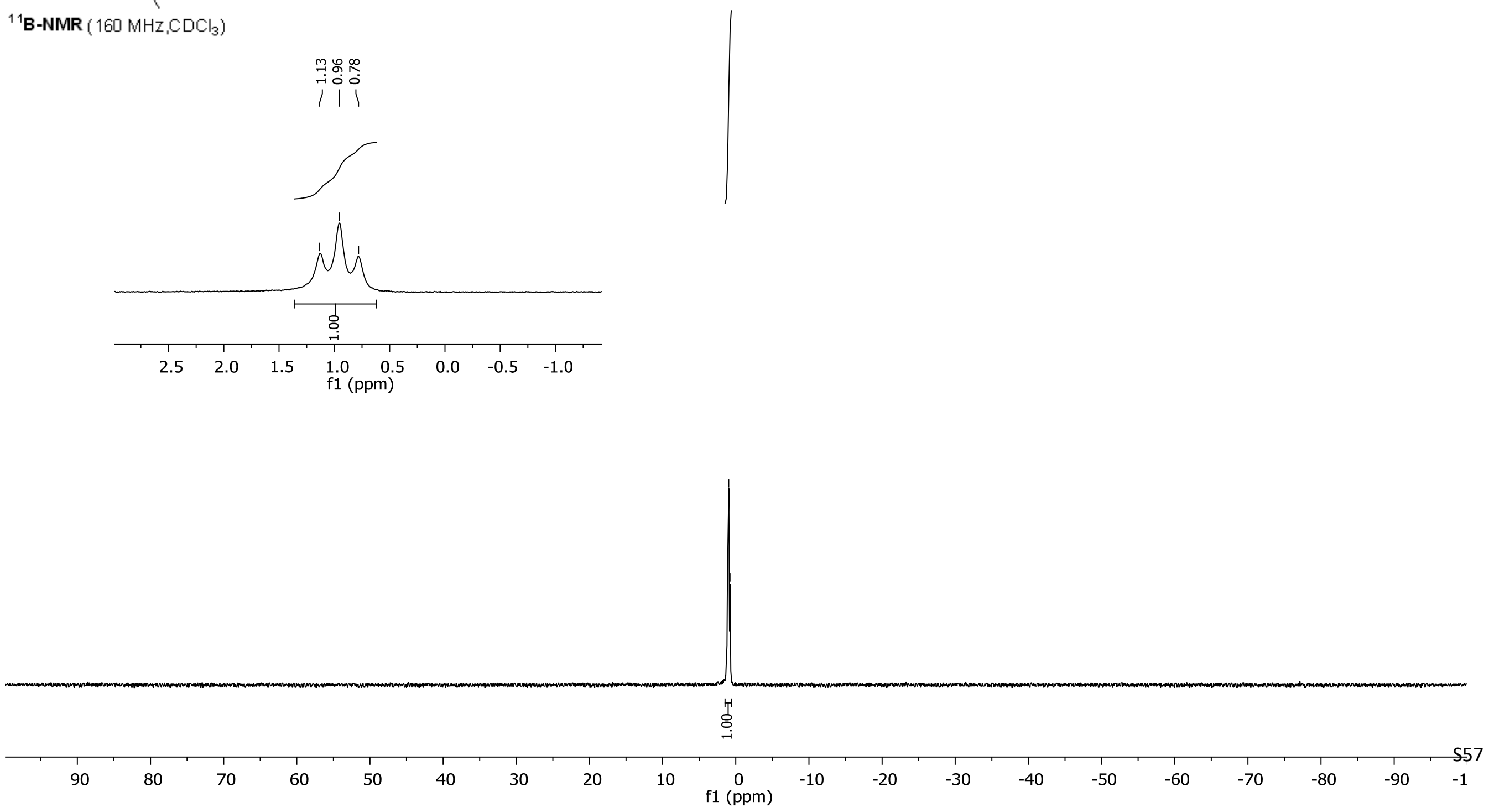


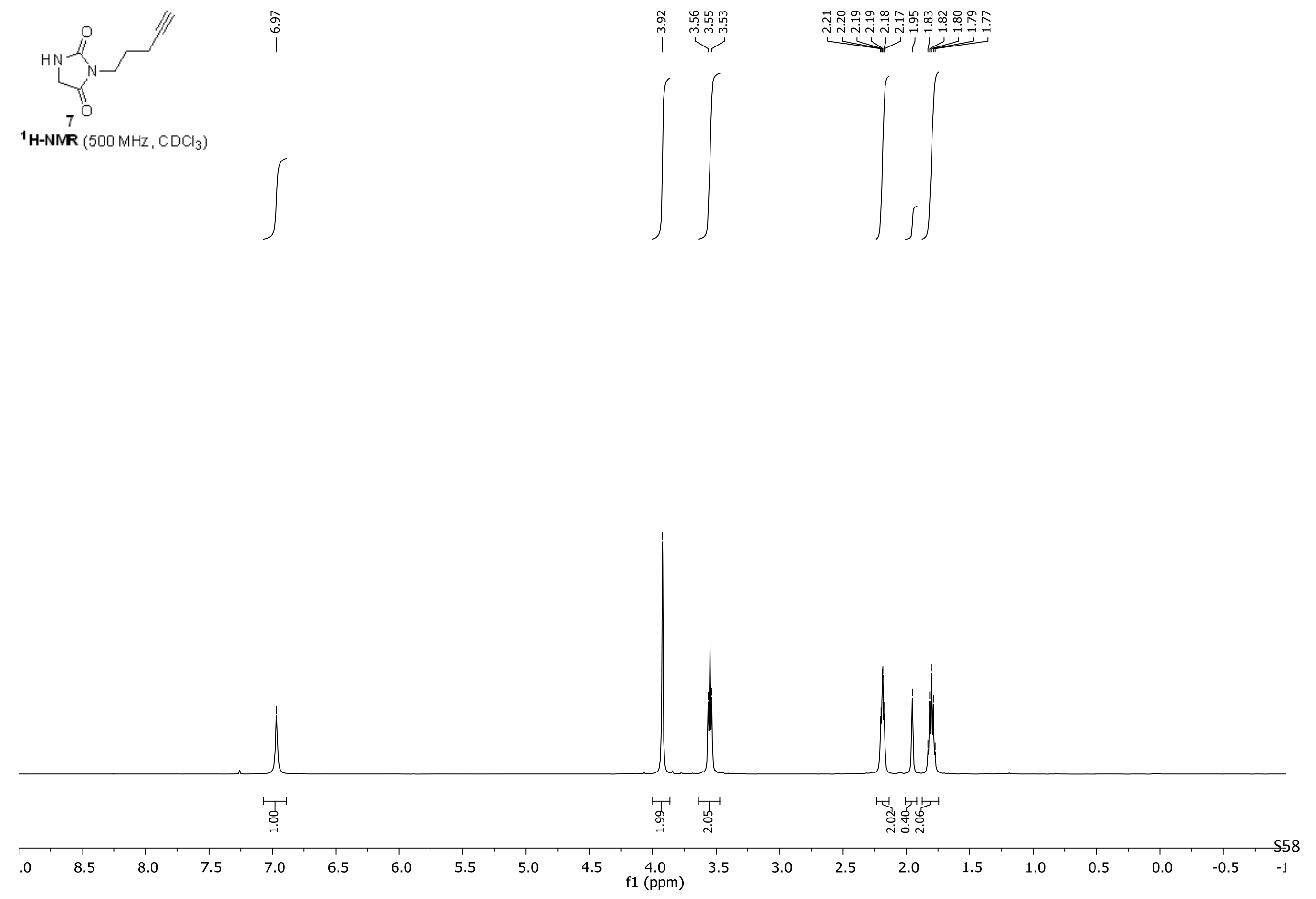




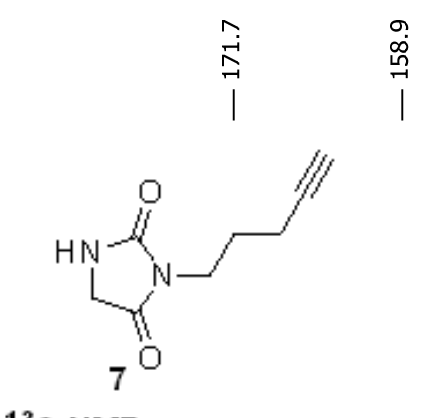

${ }^{13} \mathrm{C}$-NMR $\left(126 \mathrm{MHz}_{1} \mathrm{CDCl}_{3}\right)$
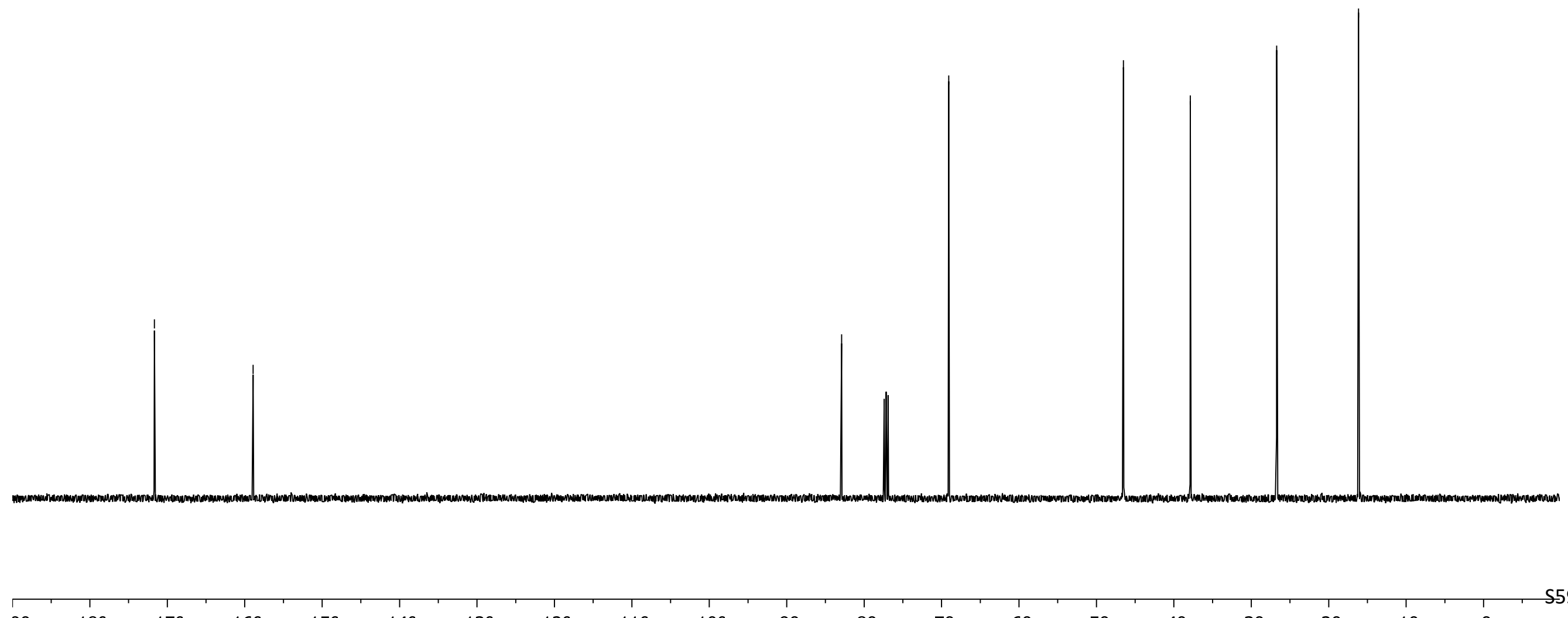

160

150

140

130

120

110

100

90

80

70

60

50

40

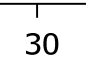

20

10 


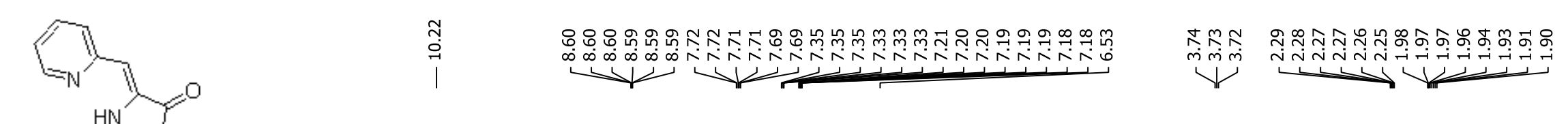

${ }^{1} \mathrm{H}-\mathrm{NMR}\left(500 \mathrm{MHz}_{1} \mathrm{CDCl}_{3}\right)$
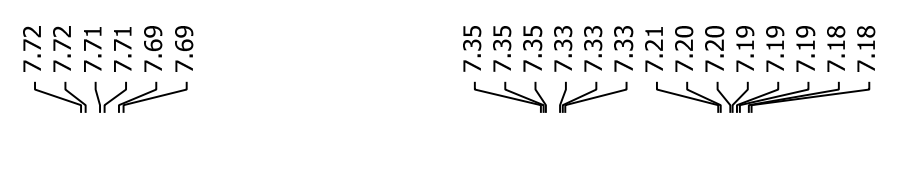

(a)
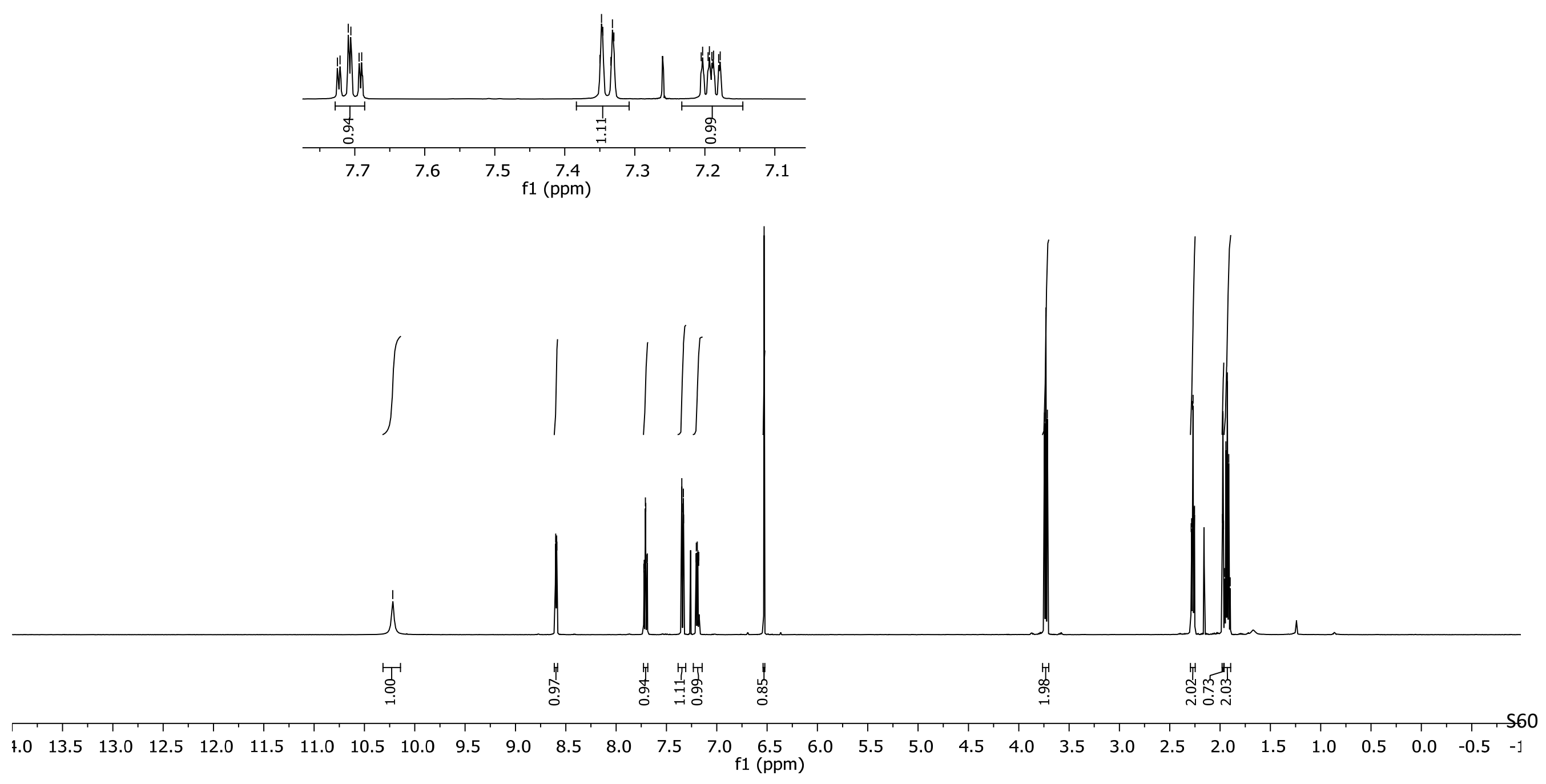


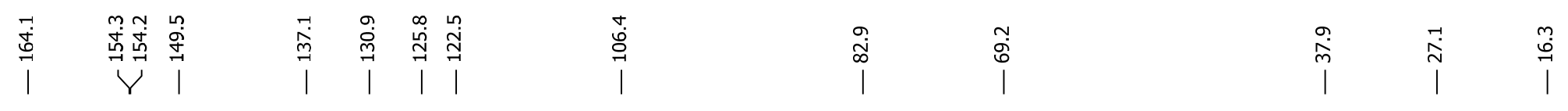

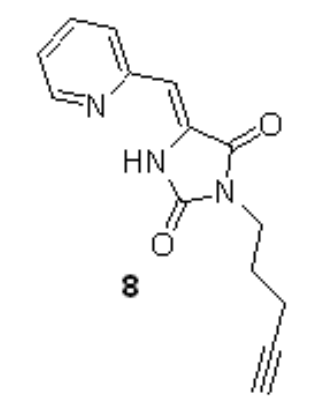

${ }^{13} \mathrm{C}-\mathrm{NMR}\left(126 \mathrm{MHz}, \mathrm{CDCl}_{3}\right)$
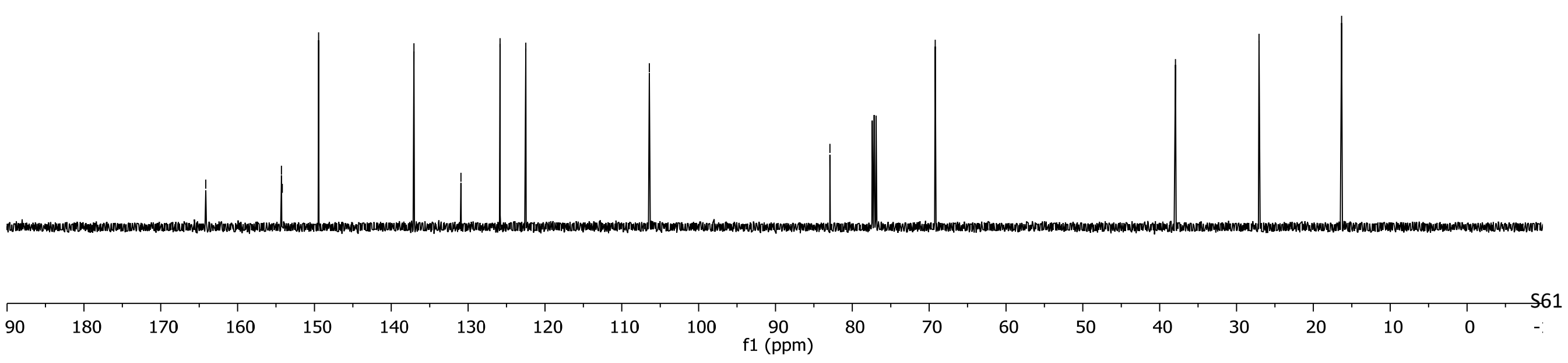
公

\section{돈

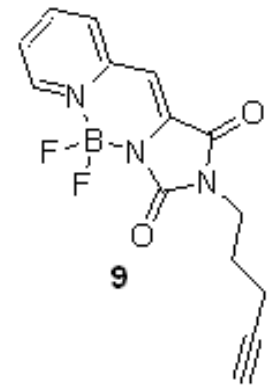

${ }^{1} \mathrm{H}-\mathrm{NMR}\left(500 \mathrm{MHz}, \mathrm{CDCl}_{3}\right)$

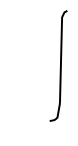

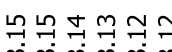

is
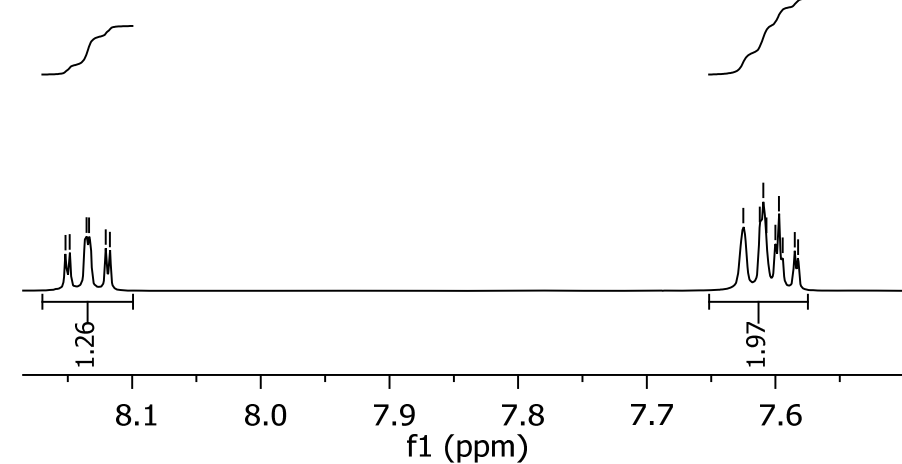

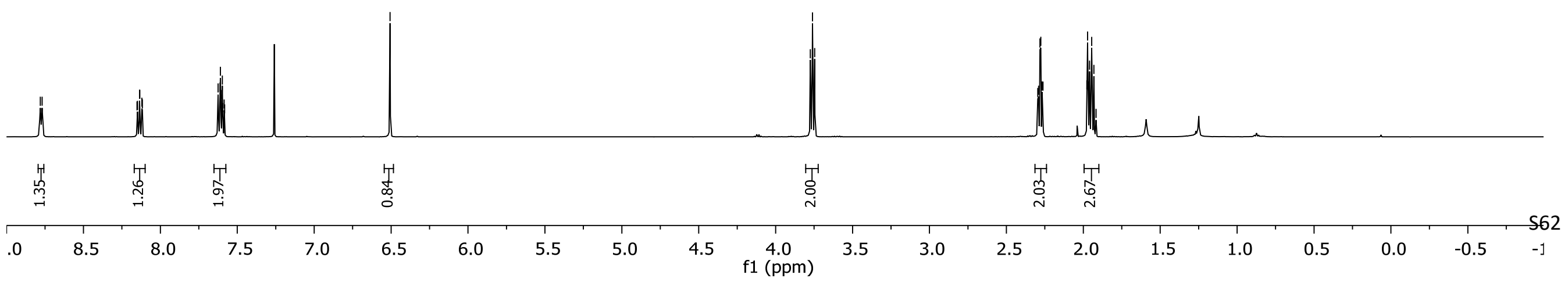

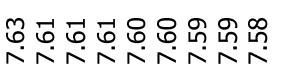

$\longrightarrow$
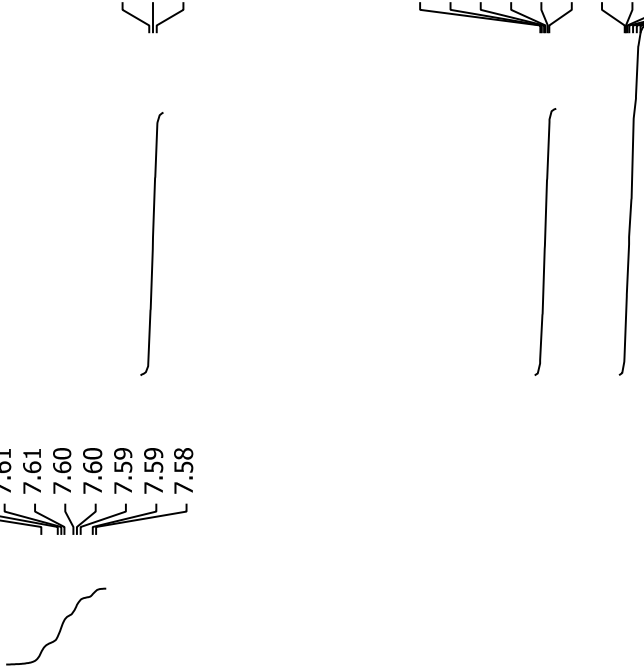


li

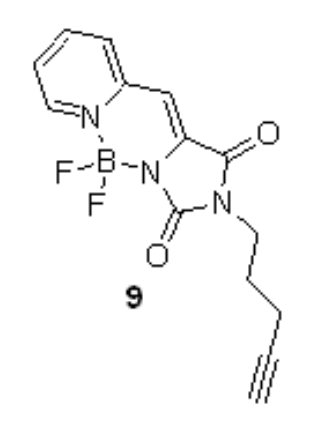

${ }^{13} \mathrm{C}-\mathrm{NMR}\left(126 \mathrm{MHz}, \mathrm{CDCl}_{3}\right)$
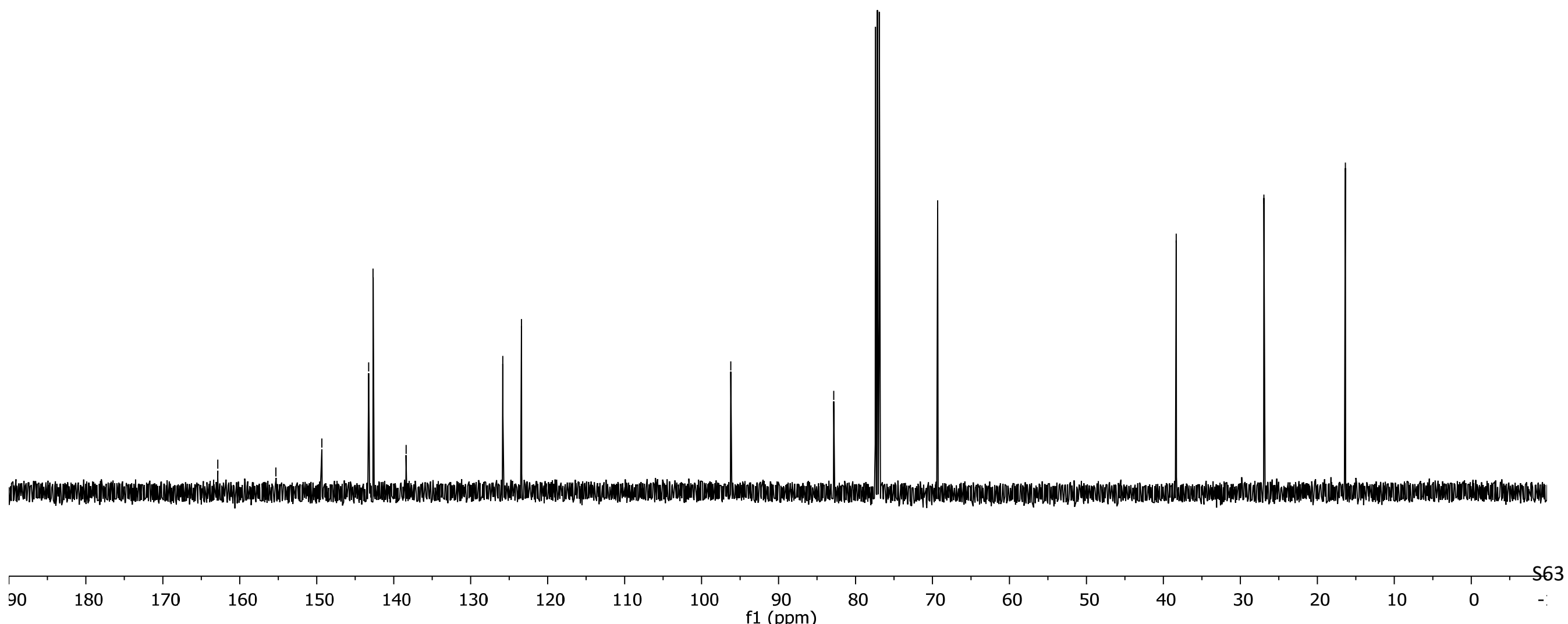


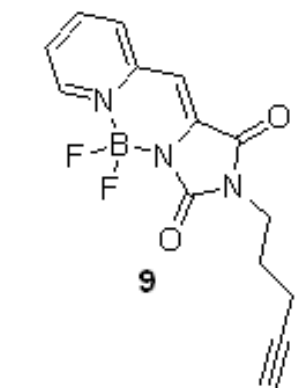

${ }^{19} \mathrm{~F}-\mathrm{NMR}\left(282 \mathrm{MHz}, \mathrm{CDCl}_{3}\right)$
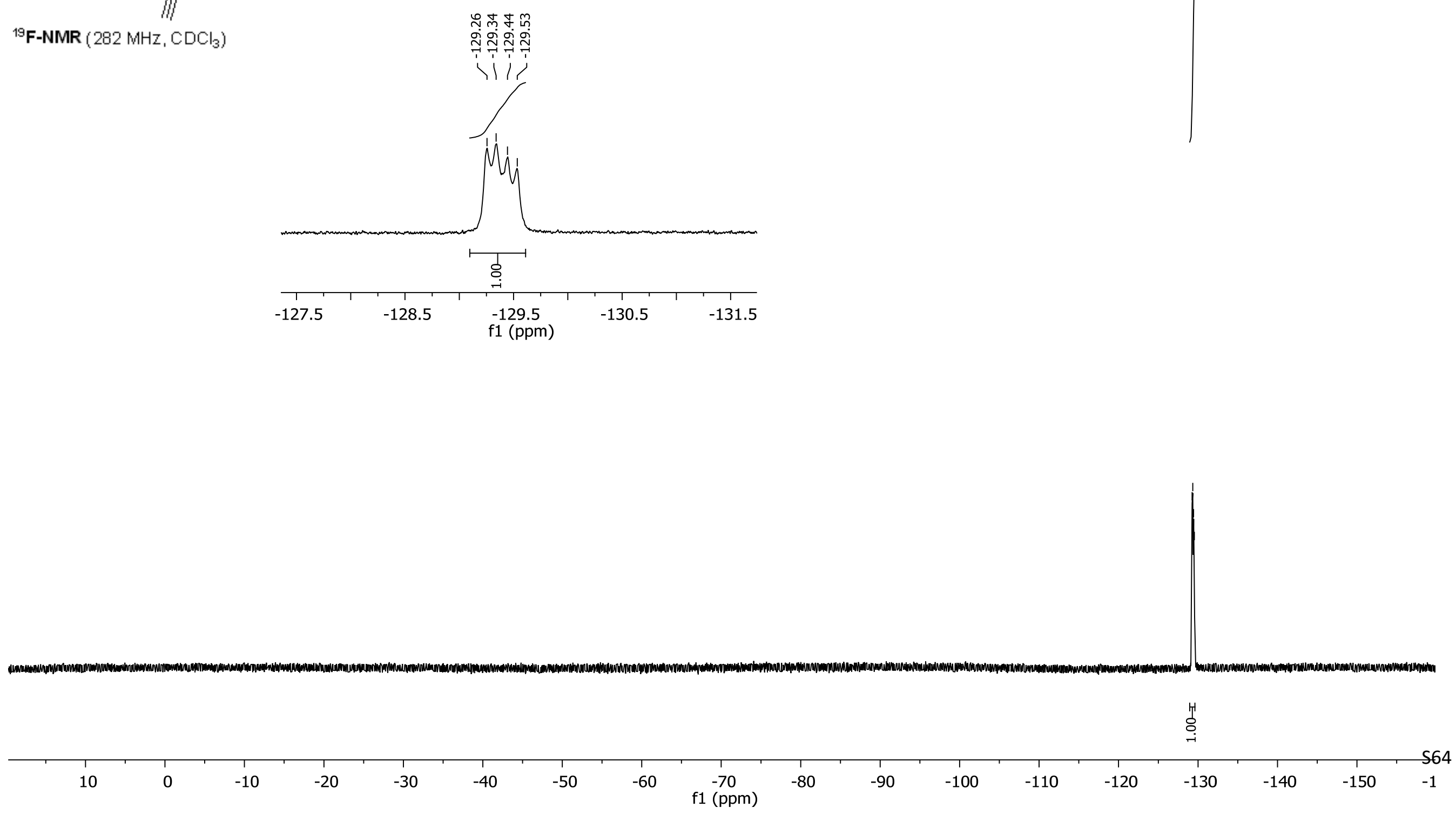


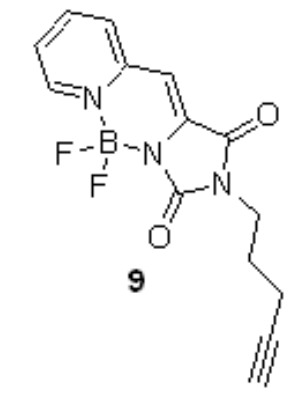

${ }^{11}$ B -NMR $\left(160 \mathrm{MHz}_{1} \mathrm{CDCl}_{3}\right)$

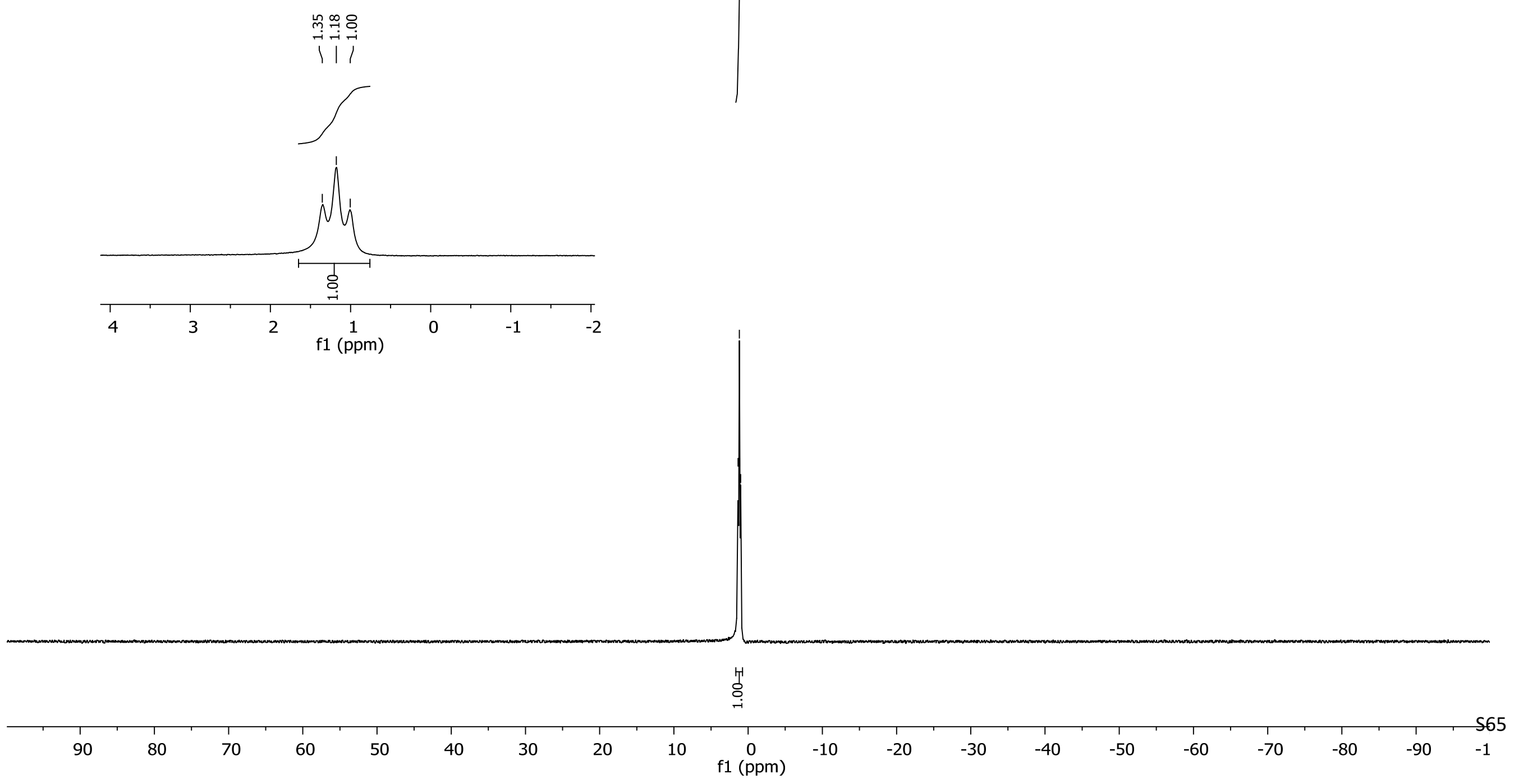




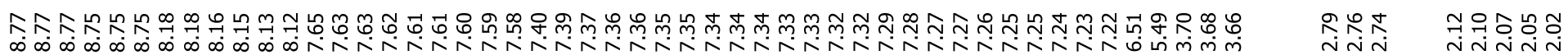

$\underbrace{\infty} \underbrace{\infty}$

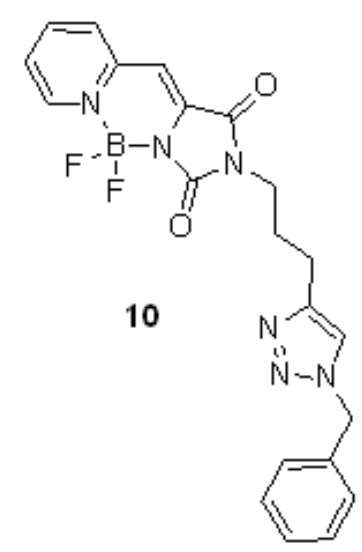

${ }^{1}$ H-NMR $\left(300 \mathrm{MHz}, \mathrm{CDCl}_{3}\right)$



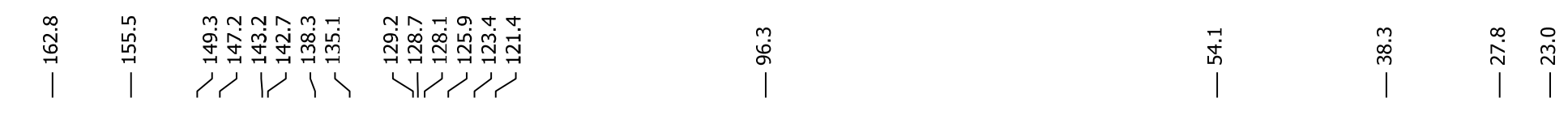

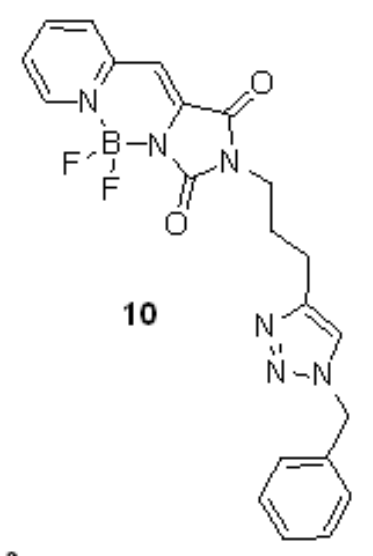

${ }^{13} \mathrm{C}-\mathrm{NMR}\left(75 \mathrm{MHz}_{1} \mathrm{CDCl}_{3}\right)$
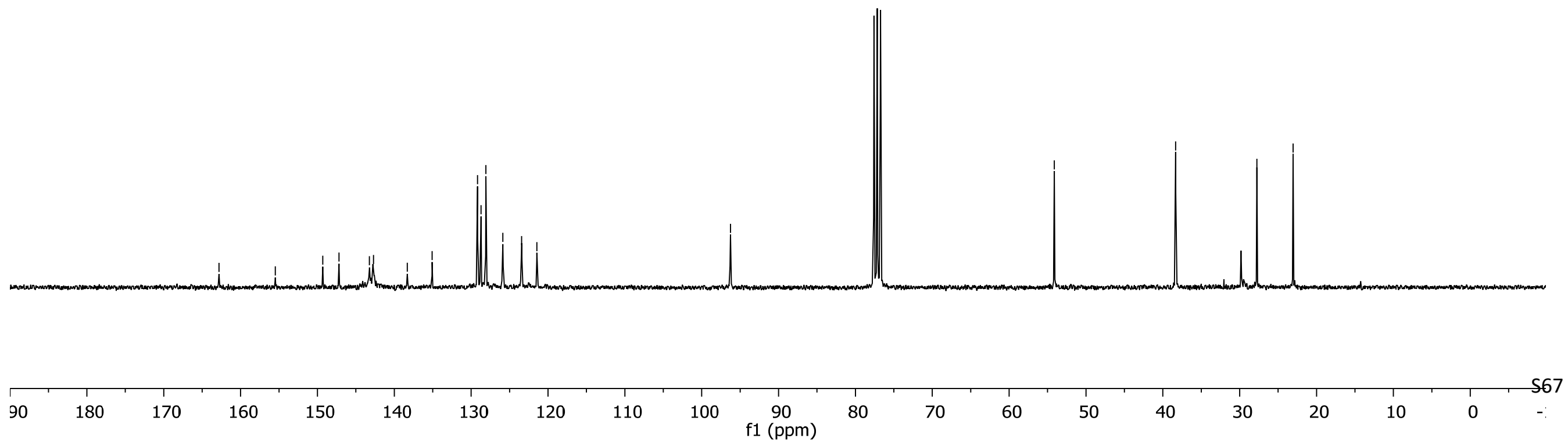

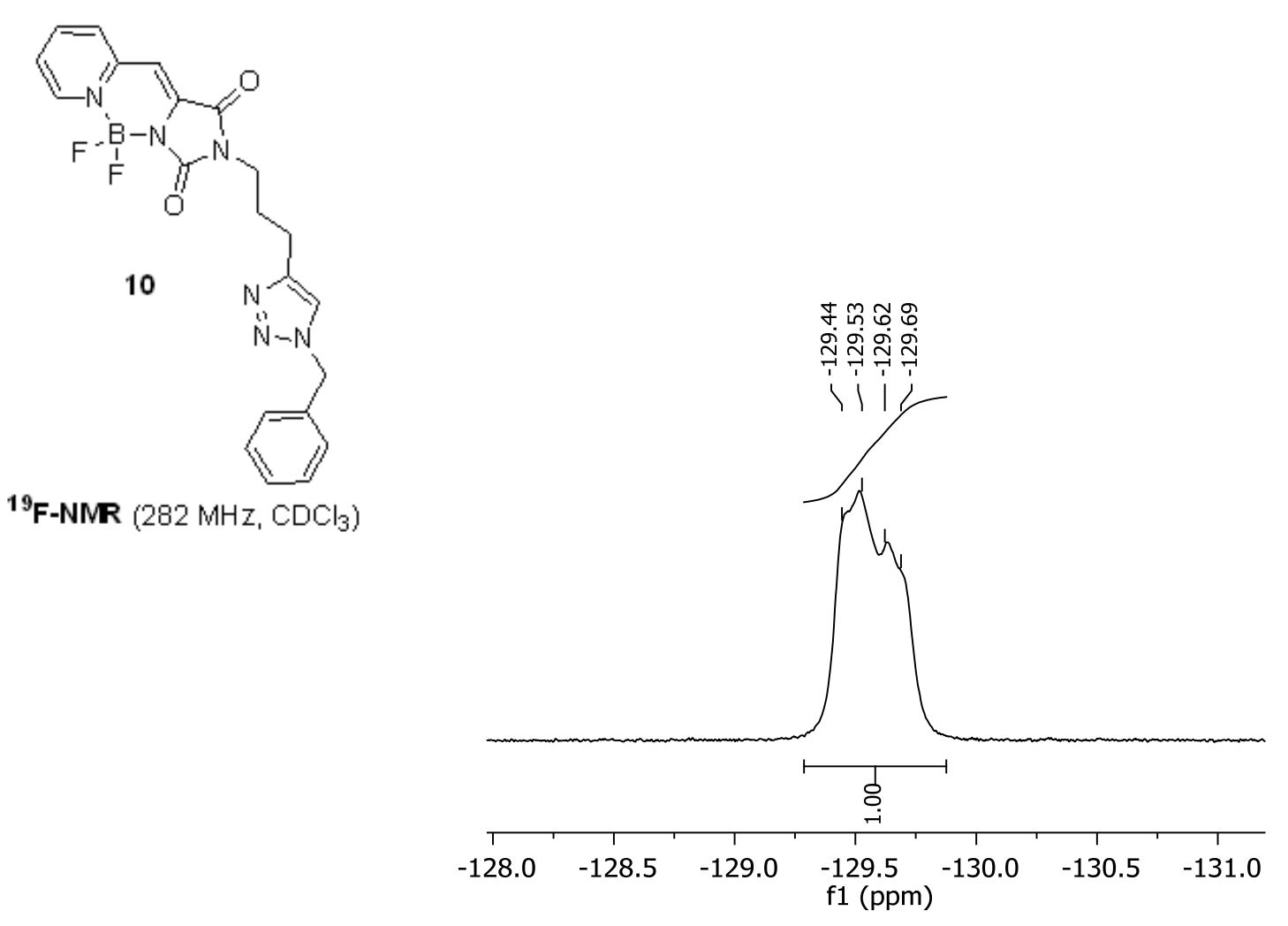

${ }^{19}$ F-NMR (282 MHz, CDCl $)$ 



${ }^{11}$ B-NMR $\left(160 \mathrm{MHz}, \mathrm{CDCl}_{3}\right)$
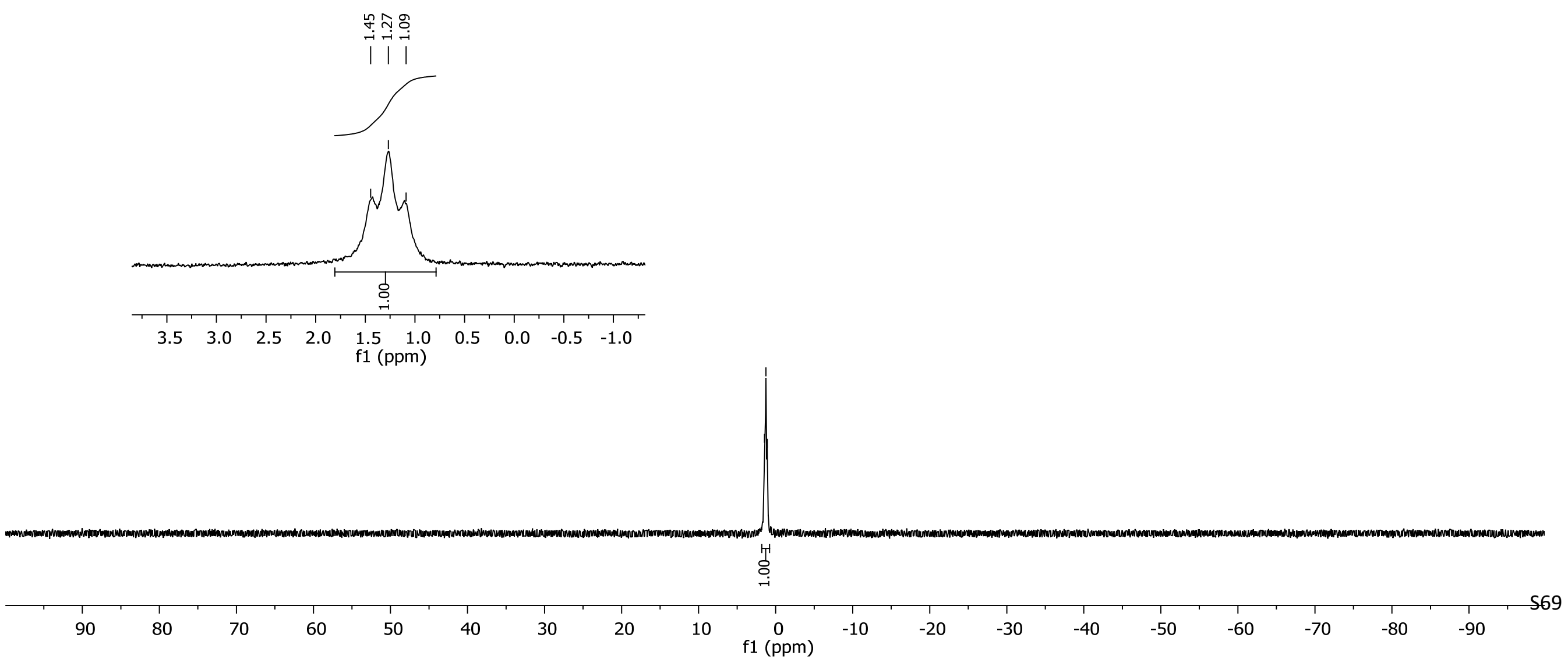


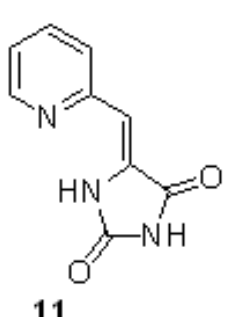

11

${ }^{1} \mathrm{H}-\mathrm{NMR}\left(500 \mathrm{MHz}, \mathrm{MeOD}-d_{4}\right)$

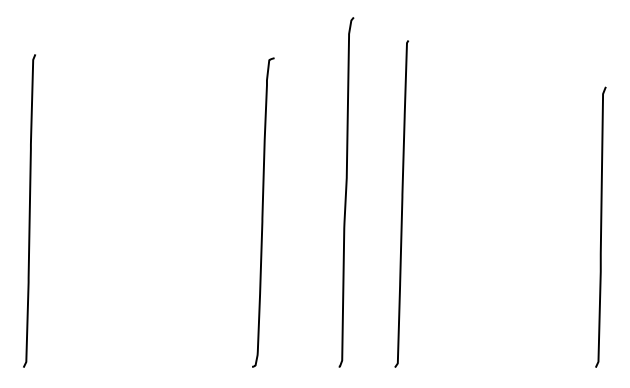

雚

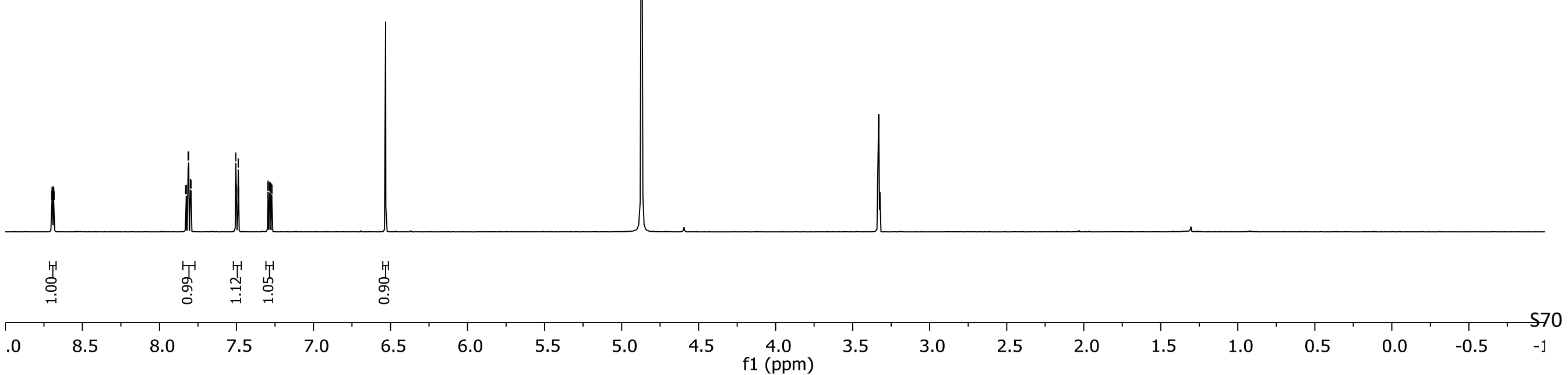

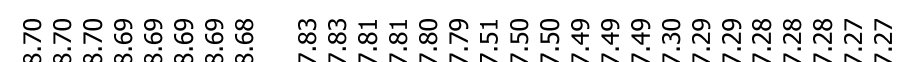
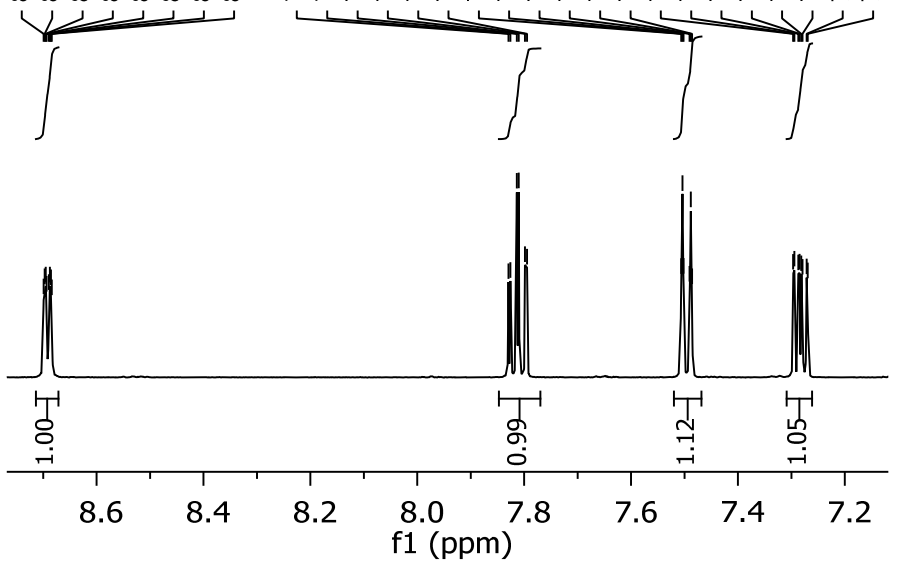

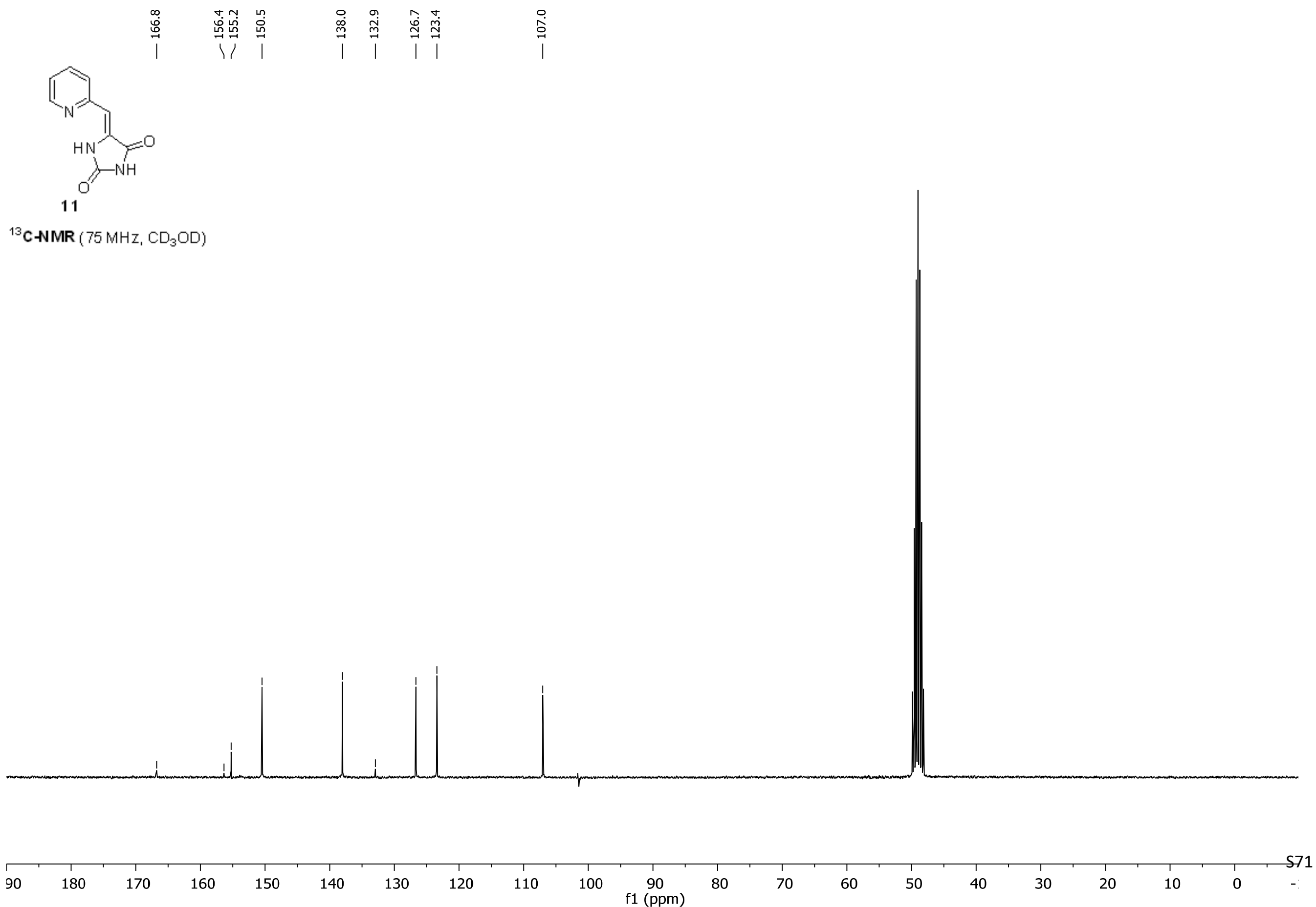


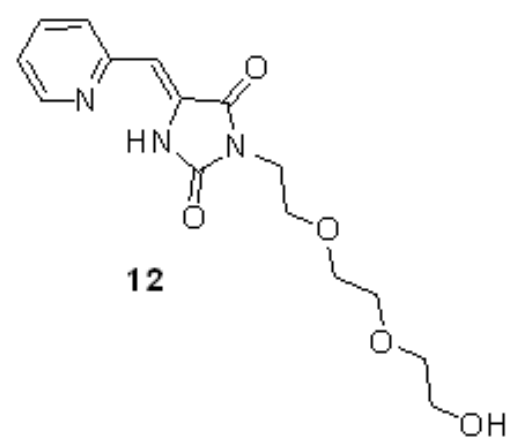

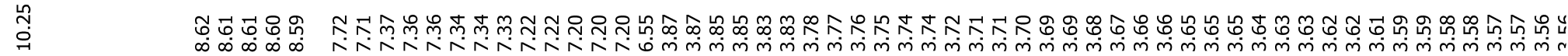

${ }^{1} \mathrm{H}-\mathrm{NMR}\left(500 \mathrm{MHz}, \mathrm{CDCl}_{3}\right)$

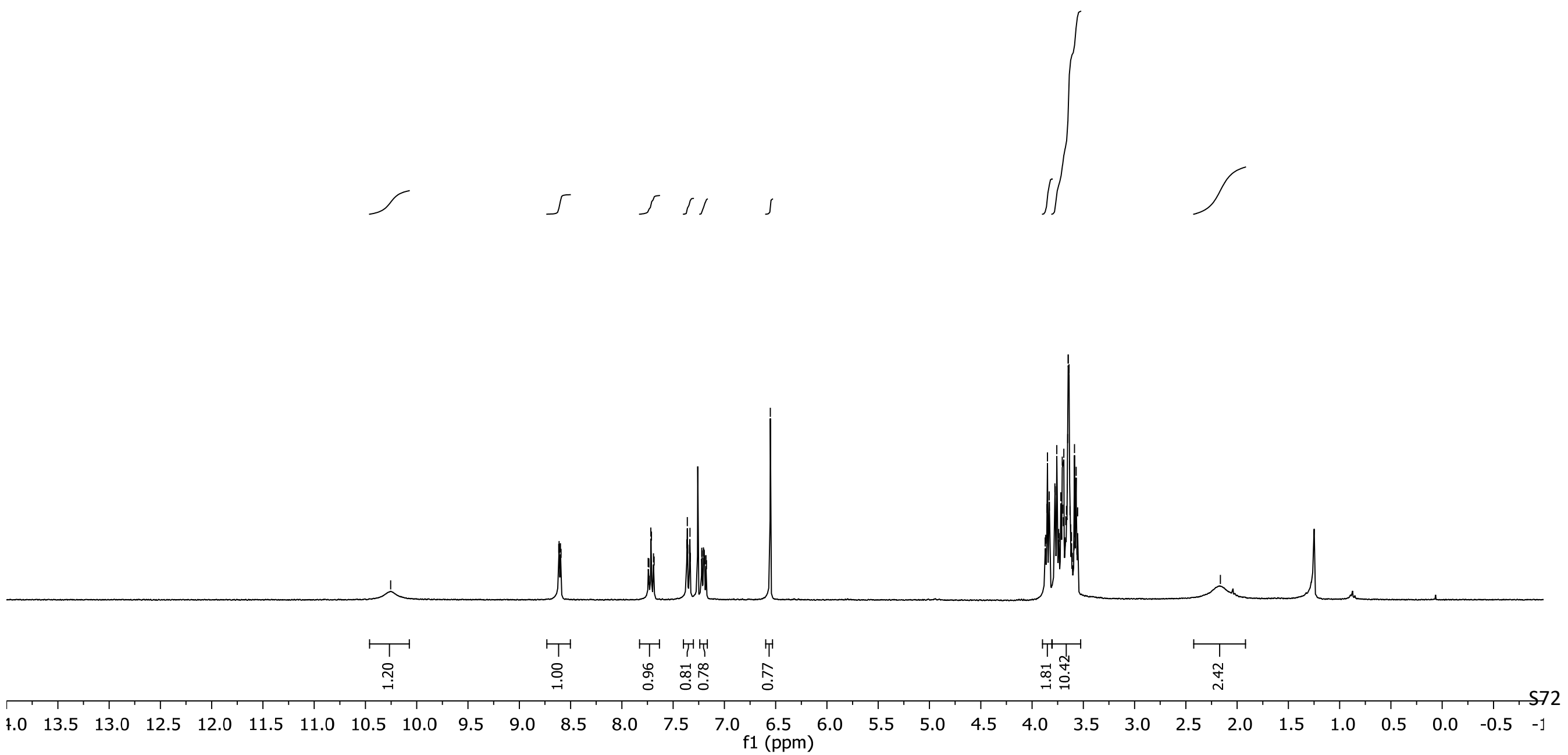




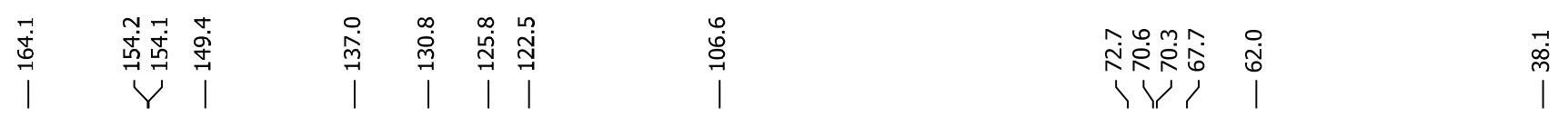

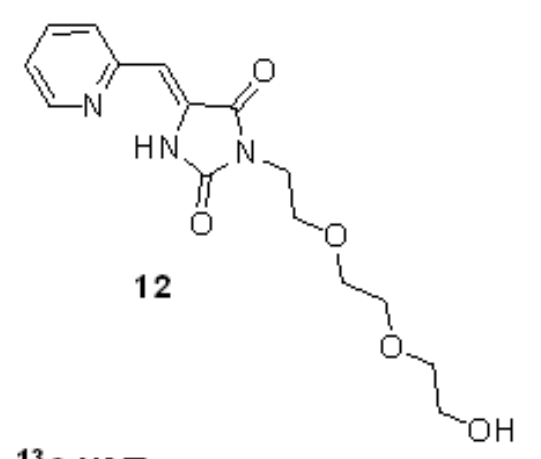

${ }^{13} \mathrm{C}-\mathrm{NMR}\left(75 \mathrm{MHz}, \mathrm{CDCl}_{3}\right)$
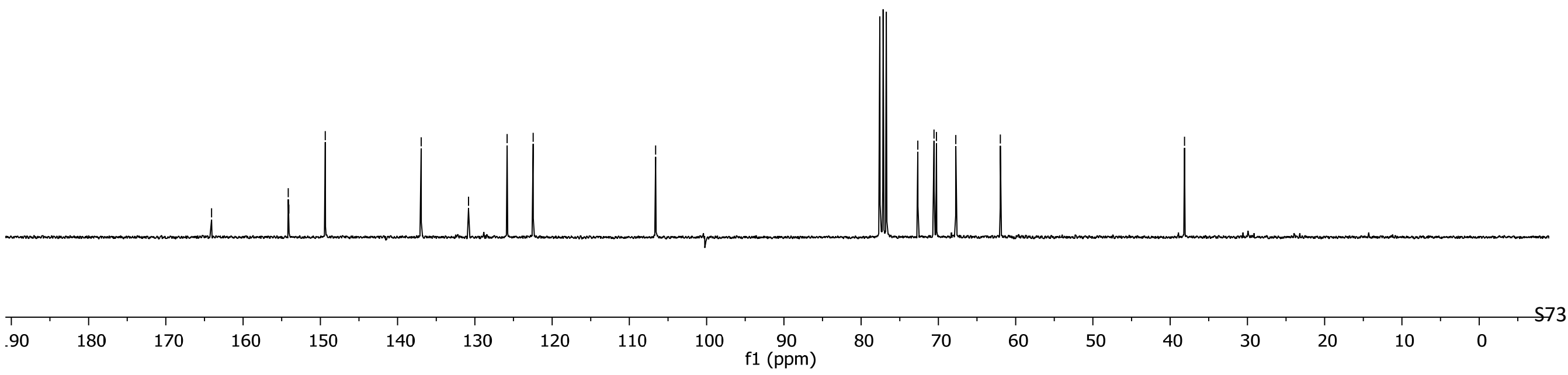


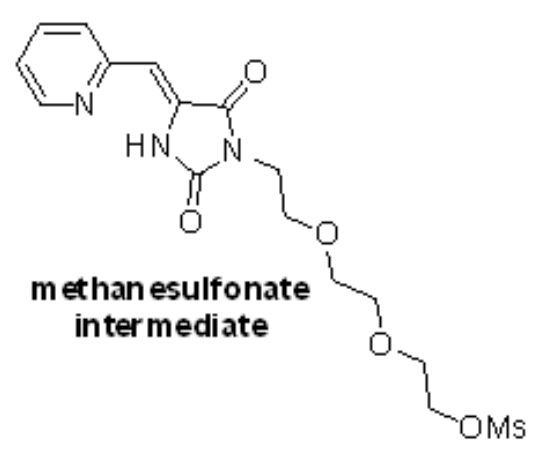

${ }^{1} \mathrm{H}-\mathrm{NMR}\left(300 \mathrm{MHz}_{1} \mathrm{CDCl}_{3}\right)$

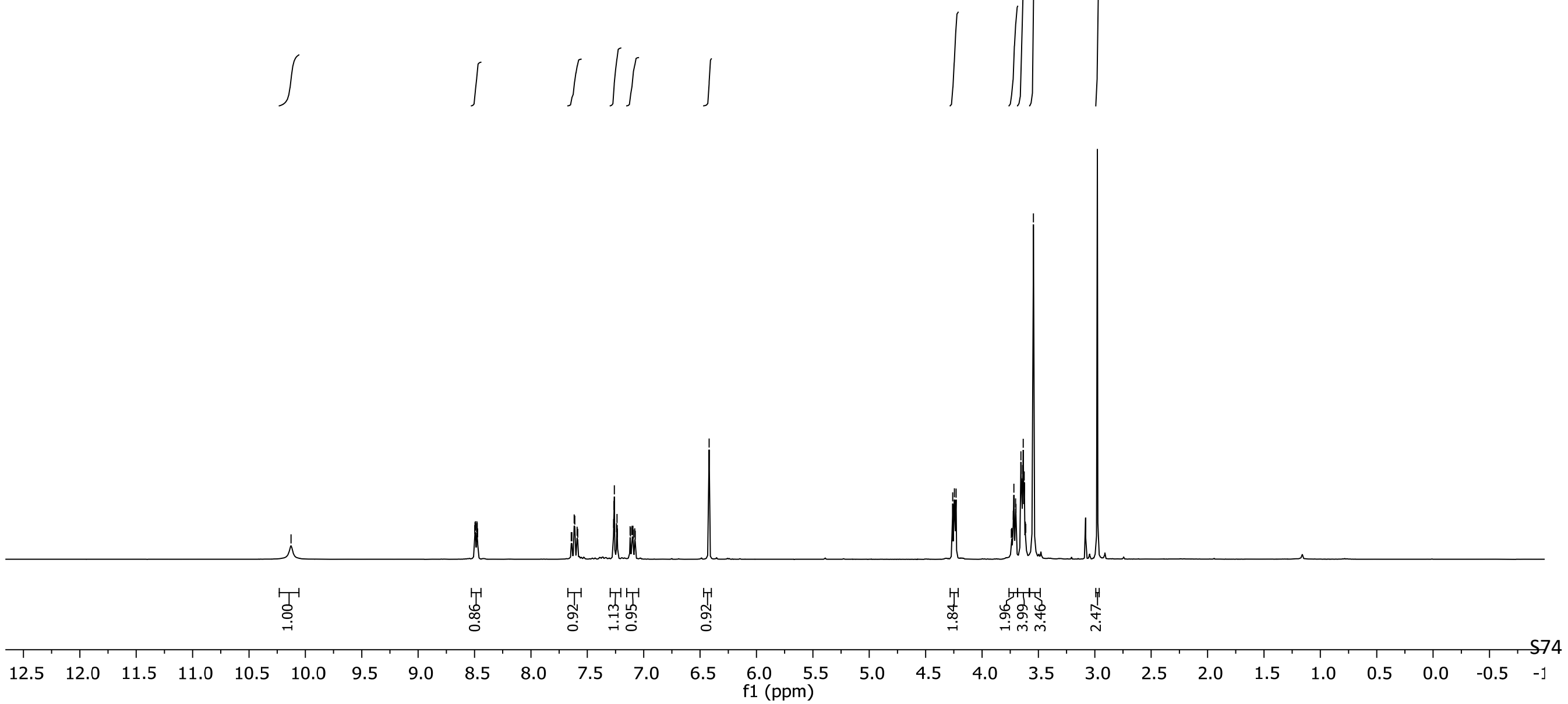



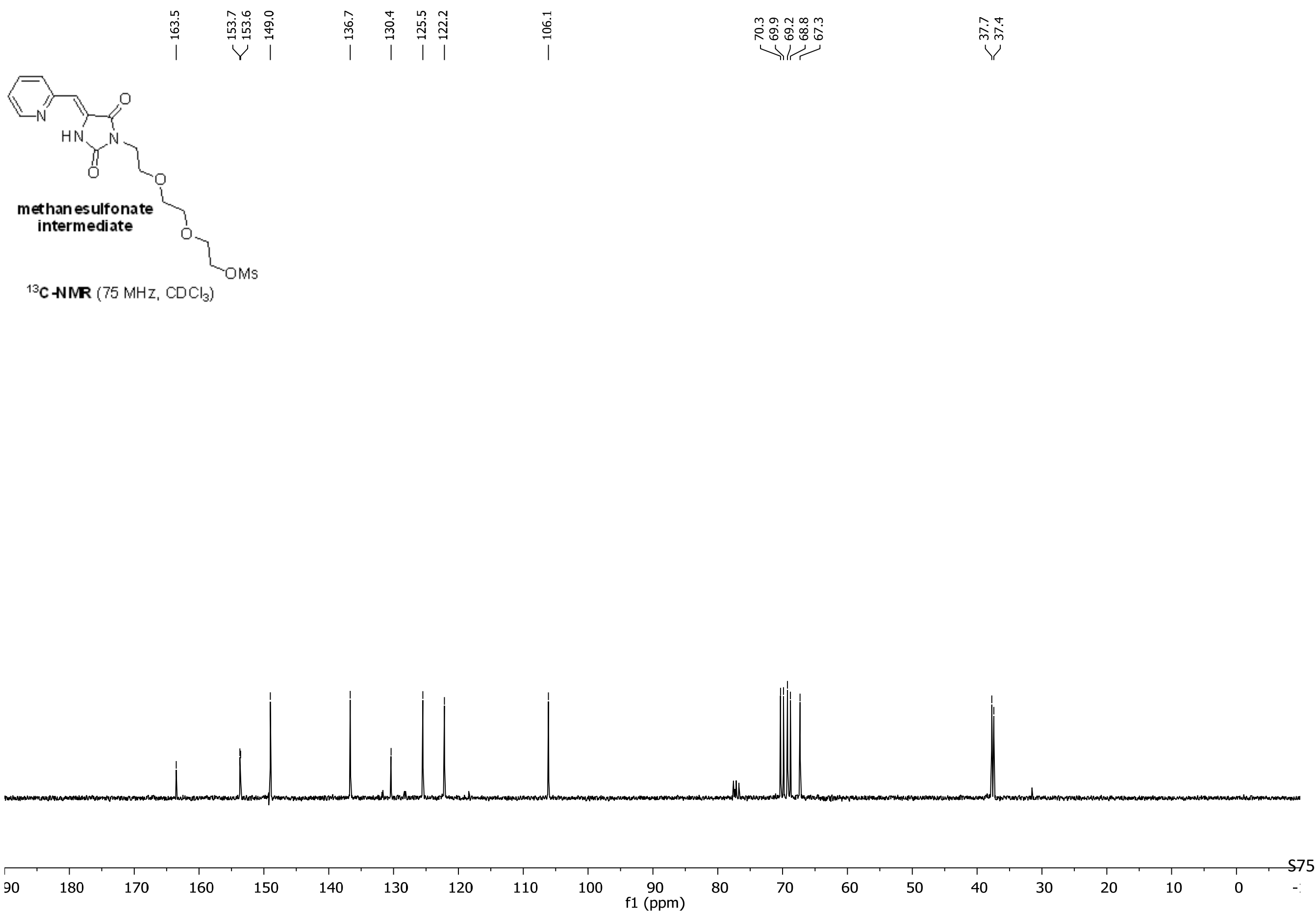

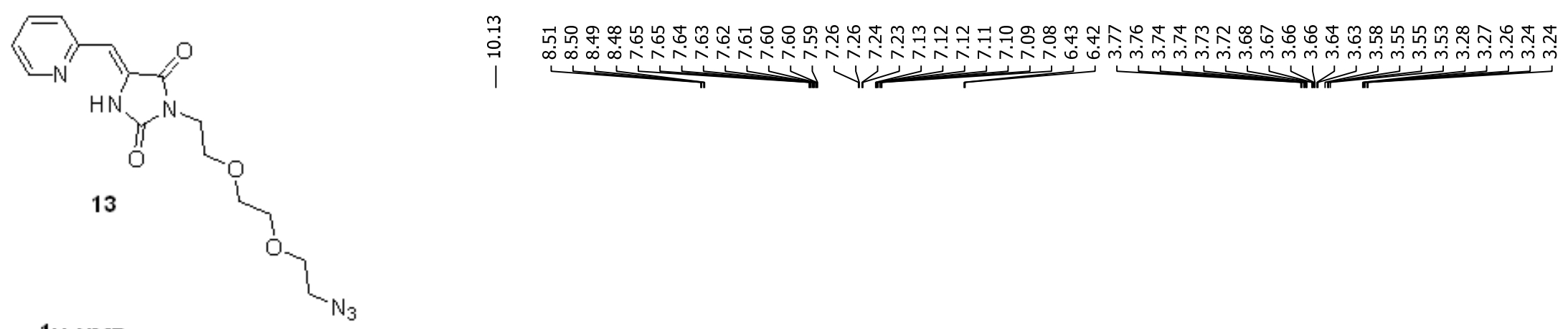

${ }^{\mathbf{H}} \mathrm{H}-\mathrm{NMR}\left(300 \mathrm{MHz}, \mathrm{CDCl}_{3}\right)$
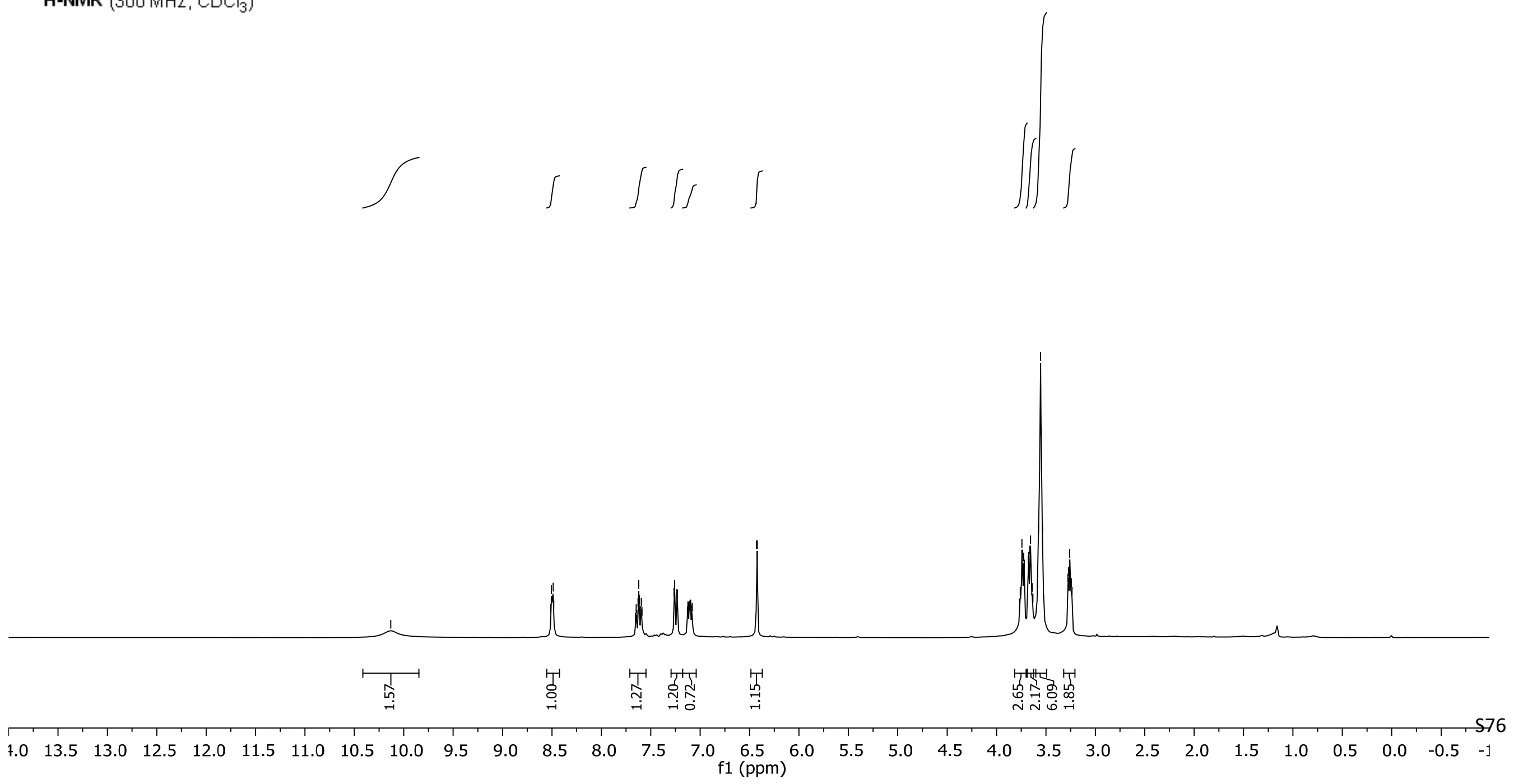


|

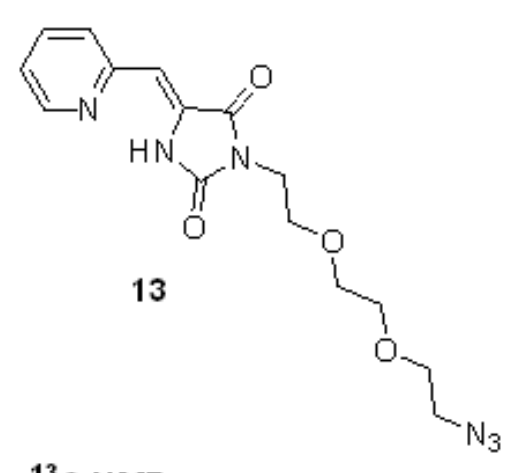

${ }^{13} \mathrm{C}-\mathrm{NMR}\left(75 \mathrm{MHz}, \mathrm{CDCl}_{3}\right)$
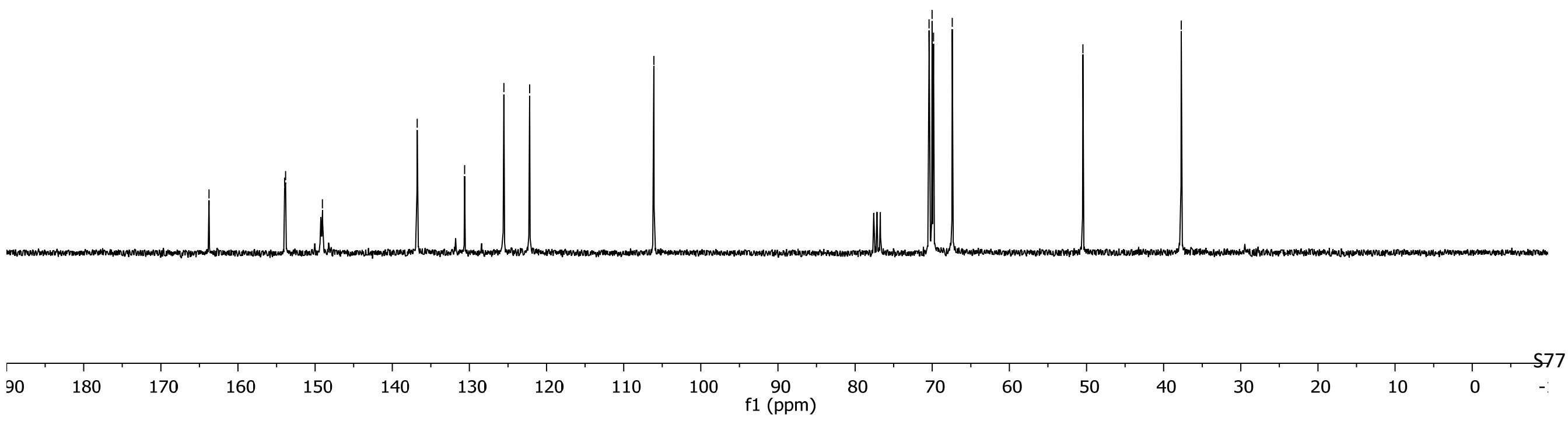


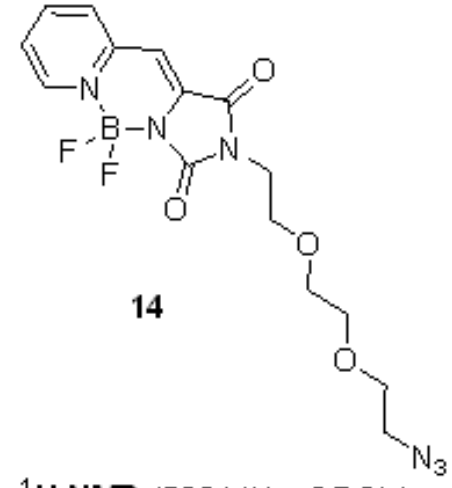

H-NMR $\left(500 \mathrm{MHz}, \mathrm{CDCl}_{3}\right)$
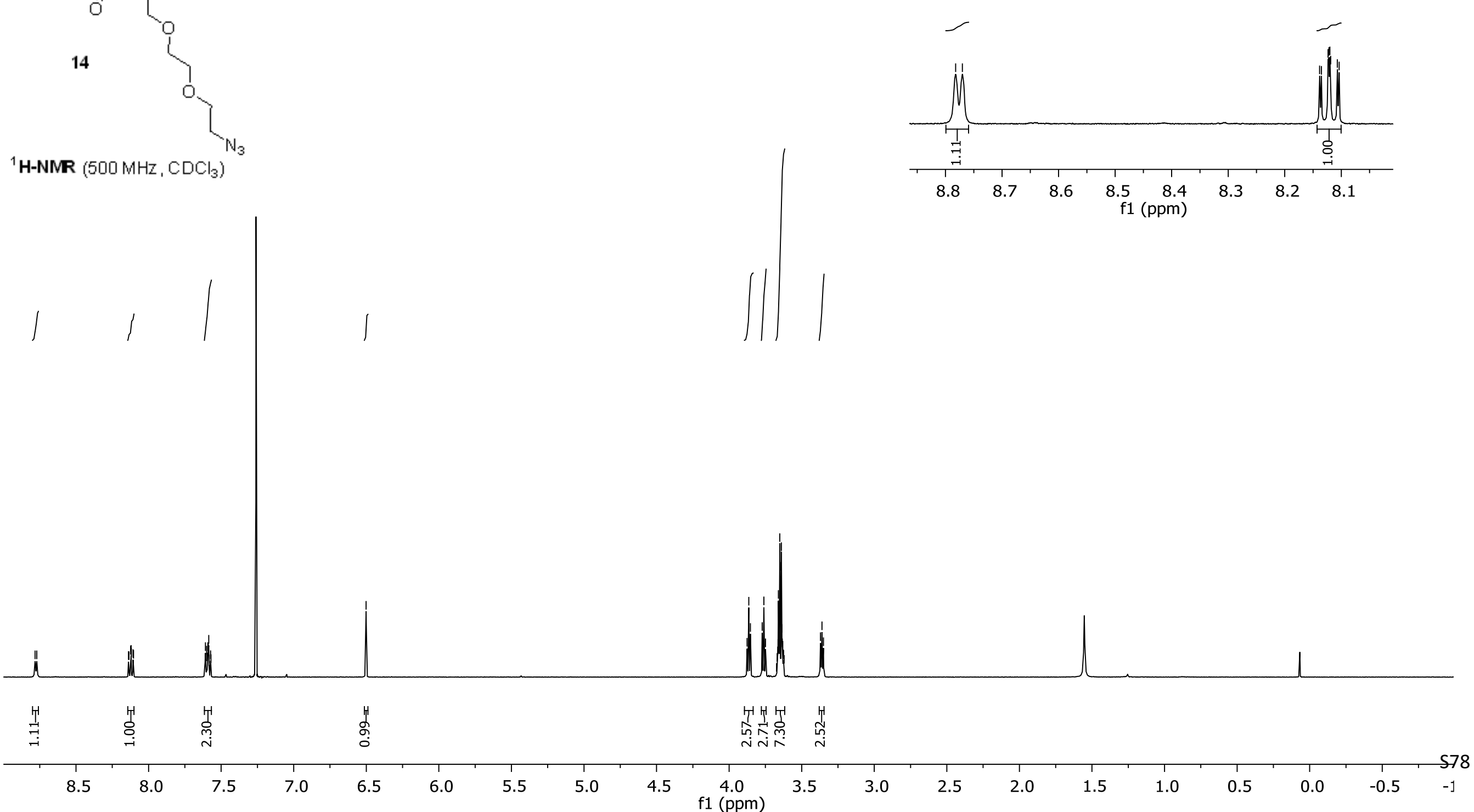


$$
\text { | }
$$

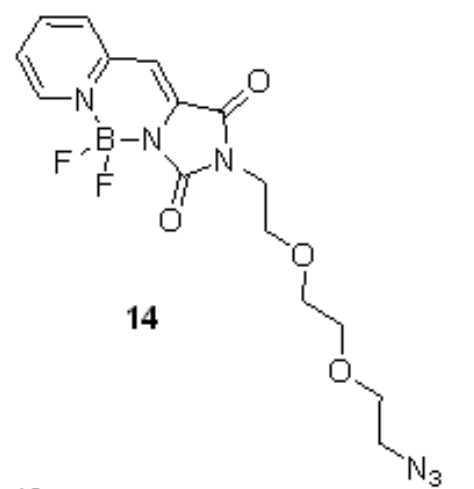

${ }^{13} \mathrm{C}-\mathrm{NMR}\left(126 \mathrm{MHZ}_{1} \mathrm{CDCl}_{3}\right)$



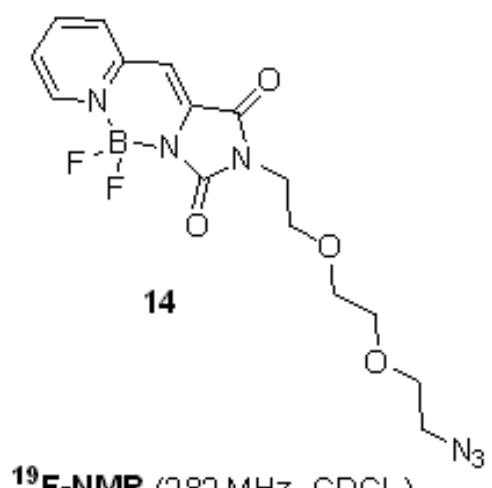

${ }^{19}$ F-NMR $\left(282 \mathrm{MHz}, \mathrm{CDCl}_{3}\right)$

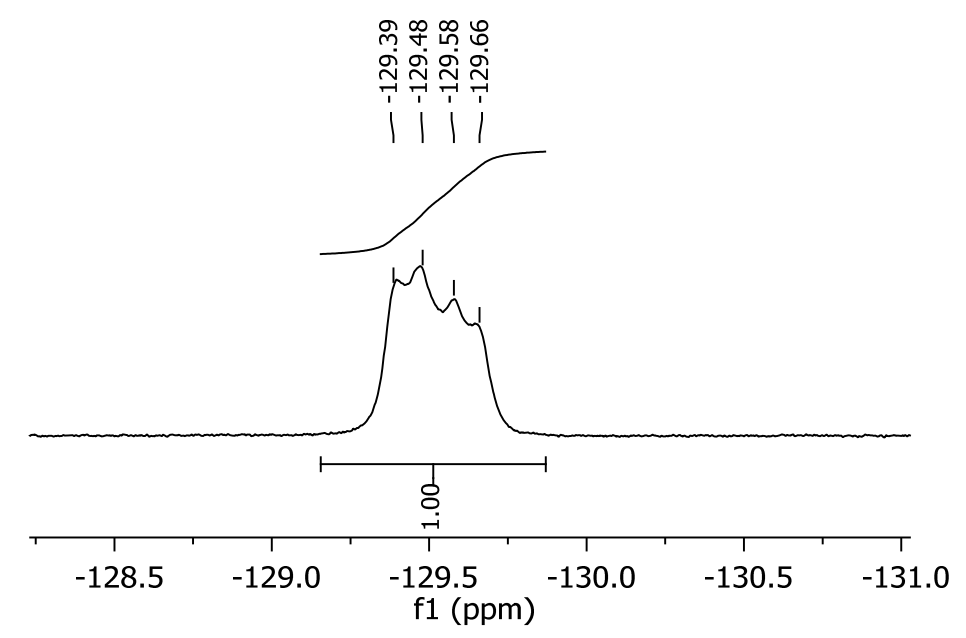




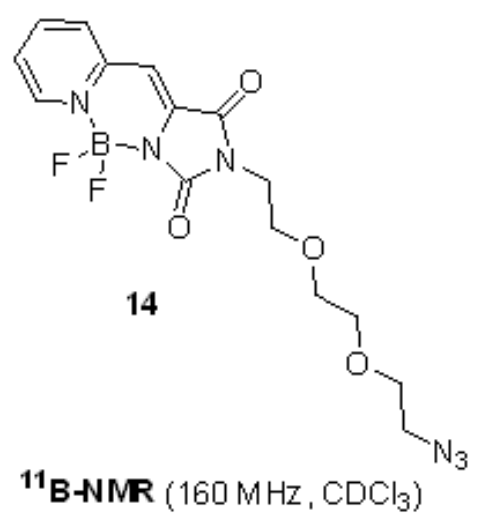

11B-NMR (160 M Hz, $\left.\mathrm{CDCl}_{3}\right)$
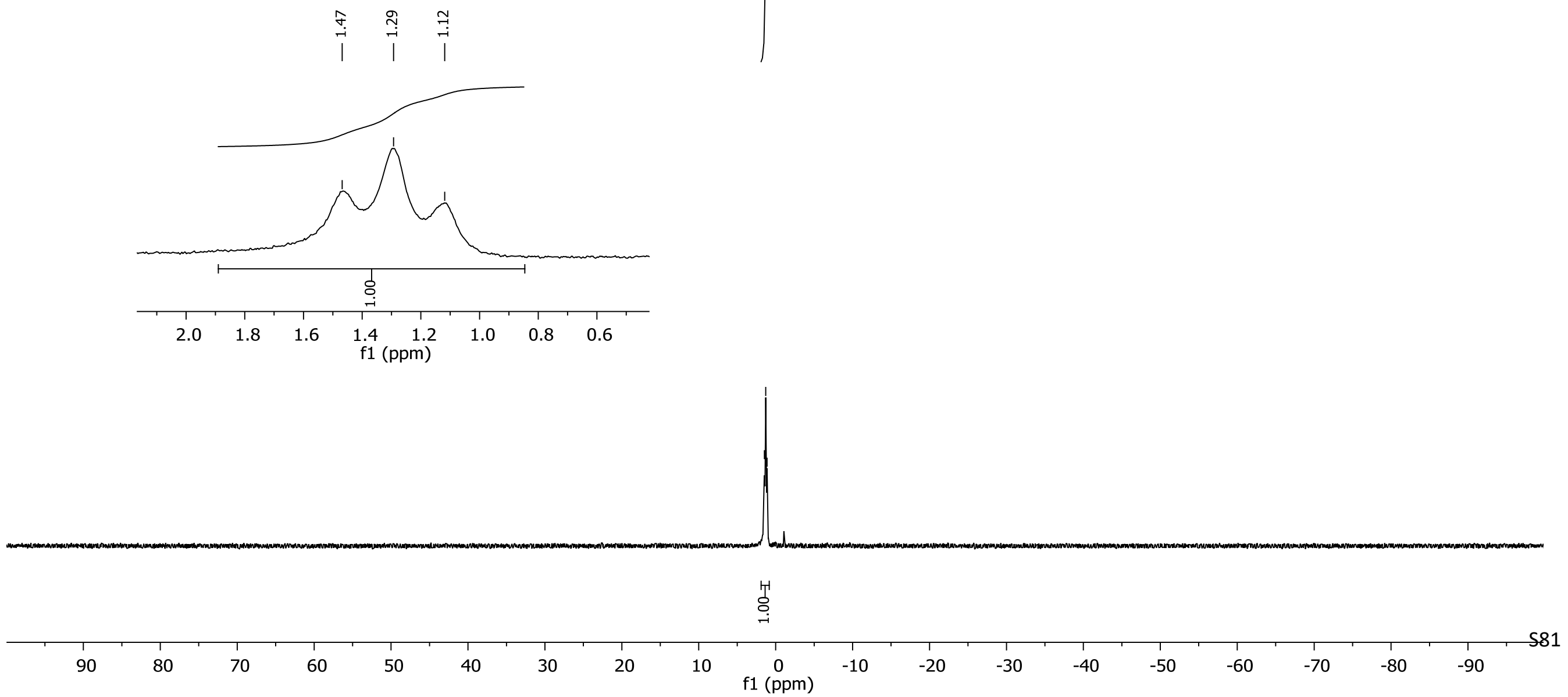